\title{
Analysis of Millimeter-Wave Networks: Blockage, Antenna Directivity, Macrodiversity, and Interference
}

\author{
Enass Hriba \\ West Virginia University, efhriba@mix.wvu.edu
}

Follow this and additional works at: https://researchrepository.wvu.edu/etd

Part of the Electrical and Electronics Commons, Signal Processing Commons, and the Systems and Communications Commons

\section{Recommended Citation}

Hriba, Enass, "Analysis of Millimeter-Wave Networks: Blockage, Antenna Directivity, Macrodiversity, and Interference" (2021). Graduate Theses, Dissertations, and Problem Reports. 10348.

https://researchrepository.wvu.edu/etd/10348

This Dissertation is protected by copyright and/or related rights. It has been brought to you by the The Research Repository @ WVU with permission from the rights-holder(s). You are free to use this Dissertation in any way that is permitted by the copyright and related rights legislation that applies to your use. For other uses you must obtain permission from the rights-holder(s) directly, unless additional rights are indicated by a Creative Commons license in the record and/ or on the work itself. This Dissertation has been accepted for inclusion in WVU Graduate Theses, Dissertations, and Problem Reports collection by an authorized administrator of The Research Repository @ WVU.

For more information, please contact researchrepository@mail.wvu.edu. 


\title{
Analysis of Millimeter-Wave Networks: Blockage, Antenna Directivity, Macrodiversity, and Interference
}

\author{
Enass Hriba \\ Dissertation submitted to the \\ College of Engineering and Mineral Resources \\ at West Virginia University \\ in partial fulfillment of the requirements \\ for the degree of \\ Ph.D. \\ in \\ Electrical Engineering \\ Matthew C. Valenti, Ph.D., Chair \\ Robert Mnatsakanov, Ph.D. \\ Daryl S. Reynolds, Ph.D. \\ Natalia A. Schmid, Ph.D. \\ Brian D. Woerner, Ph.D. \\ Lane Department of Computer Science and Electrical Engineering \\ Morgantown, West Virginia \\ 2021
}

Keywords: Correlated Blocking, Millimeter Wave, Line-Of-Sight, Macrodiversity, Outage, Interference, Device-to-Device, Unmanned Aerial Vehicle, Cellular Network.

Copyright 2021 Enass Hriba 


\begin{abstract}
Analysis of Millimeter-Wave Networks:

Blockage, Antenna Directivity, Macrodiversity, and Interference
\end{abstract}

Enass Hriba

Due to its potential to support high data rates at low latency with reasonable interference isolation because of signal blockage at these frequencies, millimeter-wave (mmWave) communications has emerged as a promising solution for next-generation wireless networks. MmWave systems are characterized by the use of highly directional antennas and susceptibility to signal blockage by buildings and other obstructions, which significantly alter the propagation environment. The received power of each transmission depends on the direction the corresponding antennas point and whether the signal's path is line-of-sight (LOS), nonLOS (i.e., partially blocked), or completely blocked. A key challenge in modeling blocking in mmWave networks is that, in actual networks, the blocking might be correlated. Such correlation arises, for example, when single transmitter tries to broadcast to pair of receivers that are close to each other, or more generally when they have a similar angle to the transmitter. In this situation, if the first receiver is blocked, it is likely that the second one is blocked, too.

This dissertation explores four related but distinct issues associated with mmWave networks: 1) Analytical modeling of networks consisting of user devices and blockages with fixed or random, but independent, locations, 2) The careful characterization of correlated blocking and analysis of its impact on the performance of mmWave networks, 3) The proposed use of macrodiversity as an important strategy to mitigating correlated blocking in mmWave networks and the corresponding analysis, and 4) The proposed use of networks of unmanned aerial vehicles (UAVs) to provide connectivity in urban deployments.

This work provides insight into the performance of variety of applications of mmWave communications, ranging from wireless personal area networks (WPAN), device-to-device networks, traditional terrestrial, cellular networks, and the UAV-based networks where the UAVs act as the cellular base stations. A common thread throughout this dissertation is the development of new tools based on stochastic geometry and their application to modeling and analysis. The analysis presented in this dissertation is general enough to find application beyond mmWave networks, for instance the results may also be applicable to systems that use free-space optical (FSO) signaling technologies. 


\section{Acknowledgments}

Firstly, I would like to express my deepest gratitude to my research advisor Dr. Matthew C. Valenti, for the continuous support of my Ph.D study and research, for his immense knowledge, motivation, enthusiasm, and patience during the past five years. I am also very grateful to Dr.Valenti for every advice and suggestion he gave me, for all the knowledge I learned from him, for all the insightful discussions we had. I could not have imagined having a better advisor and mentor for my Ph.D study.

Also, I like to extend my gratitude to my committee members, Dr. Robert Mnatsakanov, Dr. Daryl S. Reynolds, Dr. Natalia A. Schmid, and Dr. Brian D. Woerner, who spent precious time and gave me valuable guidance to improve my work.

I also gratefully acknowledge the funding received towards my PhD from the Libyan Ministry of Higher Education Scholarship program.

Finally, I would like to thank my family, especially my husband Dr. Marwan Alkhweldi for always showing how proud he is of me, for his love and support during this journey. 


\section{Contents}

$\begin{array}{ll}\text { List of Figures } & \text { vi }\end{array}$

List of Abbreviations $\quad$ x

\begin{tabular}{lll}
\hline & Introduction & 1
\end{tabular}

1.1 Motivation and Challenges . . . . . . . . . . . . . . . . . . . . . . 1

1.2 Summary of Research Accomplishments . . . . . . . . . . . . . . . . . . . . . . . 4

1.3 Millimeter Wave: Device to Device Networks . . . . . . . . . . . . . . . . . . 6

1.4 Millimeter Wave: UAV to Ground Networks . . . . . . . . . . . . . . . . . . 7

1.5 Stochastic Geometry: A Tool for Modern Networks . . . . . . . . . . . . . . 8

1.6 Organization $\ldots \ldots \ldots \ldots \ldots$

2 Accurately Accounting for Random Blockage in Device-to-Device Millimeter$\begin{array}{ll}\text { Wave Networks } & 11\end{array}$

2.1 Introduction . . . . . . . . . . . . . . . . . . . . . . . . 11

2.2 System Model . . . . . . . . . . . . . . . . . . . . . . . . . . . . . . . . . . . . . . . . . . . . . . . . . . .

$2.3 \quad$ Conditional Outage Probability . . . . . . . . . . . . . . . . . . . . . 15

2.4 Application to mmWave . . . . . . . . . . . . . . . . . . . . . 16

2.5 Spatially Averaged Outage Probability . . . . . . . . . . . . . . . . . . . . . 22

2.6 Conclusion $\ldots \ldots \ldots \ldots \ldots$

3 The Impact of Correlated Blocking on Millimeter-Wave Personal Net$\begin{array}{ll}\text { works } & 27\end{array}$

3.1 Introduction . . . . . . . . . . . . . . . . . . . . . . . . . . . . . 27

3.2 System Model . . . . . . . . . . . . . . . . . . . . . . . . . . . . . . . . 29

3.3 SINR Outage Analysis . . . . . . . . . . . . . . . . . . . . . . . . . . . . . . . . . . . . . . . . . . . 32

3.4 Blockage Correlation Coefficient . . . . . . . . . . . . . . . . . . . . . . . . . . . 35

3.5 Antenna Directivity and Spatial Randomness . . . . . . . . . . . . . . . . 37

3.6 Conclusion . . . . . . . . . . . . . . . . . . . . . 40

4 Correlated Blocking in Millimeter-Wave Cellular Networks: Macrodiver$\begin{array}{ll}\text { sity, Outage, and Interference } & 42\end{array}$

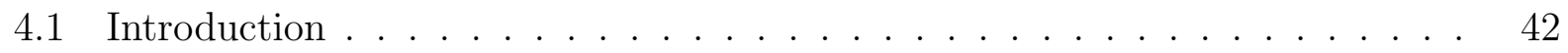

4.2 System Model . . . . . . . . . . . . . . . . . . . . . . . . . . . . . . . . . . . . . . . . . . . . . . . . . .

4.2 .1 Network Topology . . . . . . . . . . . . . . . . . . 45 
$4.2 .2 \quad$ Blockage Model . . . . . . . . . . . . . . . . . . . . 46

4.3 LOS Probability Analysis under Correlated Blocking . . . . . . . . . . . . . 48

$4.4 \quad$ SNR Distribution . . . . . . . . . . . . . . . . . . . . . . . 52

4.5 Validation with Real Data . . . . . . . . . . . . . . . . . . . . . 57

$4.6 \quad$ SINR Outage Analysis . . . . . . . . . . . . . . . . . . . . . . . . . . . . . . . 59

4.7 Conclusion . . . . . . . . . . . . . . . . . . . . . . . . 62

5 Optimization of a Millimeter-Wave UAV-to-Ground Network in Urban $\begin{array}{ll}\text { Deployments } & 64\end{array}$

5.1 Introduction . . . . . . . . . . . . . . . . . . . . . . 64

5.2 System Model . . . . . . . . . . . . . . . . . . . . . . 66

$5.3 \quad$ LoS Distribution and Outage Probability . . . . . . . . . . . . . . . . . . 69

5.4 Numerical Results . . . . . . . . . . . . . . . . . . . . . . . . . 72

5.5 Conclusion . . . . . . . . . . . . . . . . . . . . 77

6 Contributions and Further Research $\quad 81$

6.1 Summary . . . . . . . . . . . . . . . . . . . . . . . 81

6.2 Research Contributions . . . . . . . . . . . . . . . . . . . . . . . . 82

6.3 Conclusions and Future Research Directions . . . . . . . . . . . . . . . . . . 83

\begin{tabular}{|llll}
\hline A A Derivation of the PMF of a Pair of Correlated Bernoulli Variables & 87
\end{tabular}

B A Derivation of Distances to the Closest Transmitters 89

\begin{tabular}{lr}
\hline References & 90
\end{tabular} 


\section{List of Figures}

1.1 millimeter wave spectrum is the band of spectrum between $30 \mathrm{GHz}$ and 300

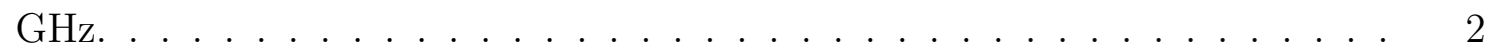

1.2 Example of blocking scenarios. . . . . . . . . . . . . . . . . . . . . 2

1.3 Several mobile wearable devices attached around a user's body a device to device network. . . . . . . . . . . . . . . . . 6

1.4 Example of UAV to ground communications . . . . . . . . . . . . . . 8

2.1 Network Topology. The $\mathrm{K}=20$ interferers are represented by the blue dots, the reference transmitter represented by the red dot, and the reference receiver represented by the red star. The yellow shaded area is the main lobe of the receiver's antenna. . . . . . . . . . . . . . . . . . . 17

2.2 An example network in the upper left portion of the figure. The curves show the outage probability for this particular network at SNR $=20 \mathrm{~dB}$. The black dots represent simulation results. In addition to the exact outage probability found using the methods of this chapter, the outage probability using the

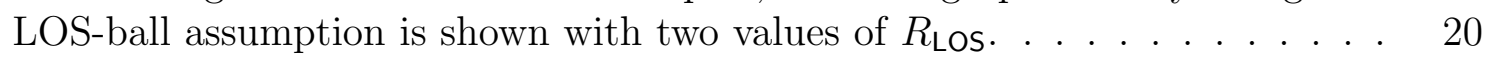

2.3 The outage probability conditioned on several network realization is plotted by dashed lines. The average outage probability over 100 network realization is plotted by the solid red line. . . . . . . . . . . . . . . . . . . 21

$2.4 \quad$ SINR coverage probability for different transmission probability $p_{\mathrm{t}} \ldots \ldots$. . . 21

2.5 CDF of spectral efficiency for different number of antenna arrays at the transmitter and the receiver . . . . . . . . . . . . . . . 22

2.6 Spatially averaged outage probability with $K=20$ randomly located interferers and SNR $=20 \mathrm{~dB}$. In addition to the exact values, the outage found using the LOS-ball approximation and two values of $R_{\text {LOS }}$ are shown. The black dots represents simulation results. . . . . . . . . . . . . . 26

3.1 Example network topology. Denoted by a red star, the receiver is located at the center of the circular region $A$. Denoted by red dots, the two interferers are in the northern part of $A$. The blocking zones of each interferer are indicated by colored rectangles, and their intersection is represented by $v$. . . . . . . . 30 
3.2 The CDF of the SINR $F_{\text {SINR }}(\beta)$ for different values of $\rho$. The thick black line shows the CDF when $\rho=0$, the dashed blue line shows the case when $\rho=$ -0.1, and the solid blue lines correspond to positive values of $\rho$ in increments

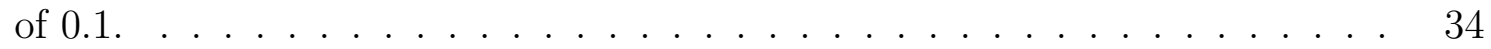

3.3 The correlation coefficient $(\rho)$ versus the angular separation between $X_{1}$ and $X_{2}\left(\theta=\left|\phi_{1}-\phi_{2}\right|\right)$ for different values of blockage width $(W)$. The black dots represent simulation results. The curves show the exact analytical expression found using the methods of this chapter. . . . . . . . . . . . . . . . 36

3.4 The correlation coefficient $(\rho)$ versus the angular separation between $X_{1}$ and $X_{2}\left(\theta=\left|\phi_{1}-\phi_{2}\right|\right)$ for different values of number of blockages $(K) . \quad \ldots . . \quad$. 37

3.5 Comparison of CDF of SINR that accounts for correlated blockage (solid blue line) against independent blockage (dotted red line). $\quad \ldots$. . . . . . . . . . . 38

$3.6 \quad$ Actual antenna model (solid red line) versus sectorized antenna model (dashed blue line). . . . . . . . . . . . . . . . . . . . . . . . . 40

3.7 Comparison of the CDF of SINR for a particular network realization with the actual antenna pattern (red curves) vs. with a sectorized antenna model (blue curves). Solid lines account for correlated blocking while dashed lines assume independent blocking. . . . . . . . . . . . . . . . . . . . . 4 41

3.8 The CDF of SINR when averaged over 1000 network network realizations. Red curves use an actual antenna pattern and blue curves use a sectorized antenna model. Solid lines account for correlated blocking while dashed lines assume independent blocking. . . . . . . . . . . . . . . . . . . . . . 41

4.1 Example network topology with two different blockage scenarios. The source transmitter is the mobile device shown in the central cell. Its signal is transmitted to its closest two base stations. The solid line indicates the link is LOS, while the dashed line indicates the link is NLOS. . . . . . . . . . . . . 46

4.2 Illustration of the blockage model. (a) Network example consisting of base stations indicated by the stars and blockages indicated by blue lines surrounding the transmitter, which is indicated by the black circle. The blockages are modeled as a line of length $\mathrm{W}$ facing the transmitter; (b) Equivalent blockage regions. $a_{1}$ and $a_{2}$ are the blockage areas, and $\mathrm{v}$ is the overlapping area. $\quad$. . 47

4.3 The correlation coefficient $(\rho)$ versus the angular separation $(\theta)$ between $X_{1}$ and $X_{2}$. . . . . . . . . . . . . . . . . . . . . . . . . . . . . . . . 50

4.4 The empirical CDF of $p_{L O S}$ over 1000 network realizations when $N=1,2$, with and without considering blockage correlation at fixed blockage width $W=0.8 . \ldots \ldots \ldots \ldots \ldots 1$

4.5 The variation of the spatially averaged $p_{L O S}$ over 1000 network realizations with respect to blockage density $\lambda_{b l}$ when $N=1,2$, with and without considering blockage correlation at fixed base station density $\lambda_{b s}=0.3$. . . . . . . . 51

4.6 The CDF of the SNR $F_{\text {SINR }}(\beta)$ using diversity combining for fixed location of $X_{1}$ and $X_{2}$ for different values of $\rho$. The dashed red line shows the CDF when $\rho=0$, and the solid blue lines correspond to positive values of $\rho$ in increments of 0.1 . 
4.7 The distribution of SNR over 1000 network realizations when $N=1,2$ using diversity combining, with and without considering blockage correlation at fixed values of blockage density $\lambda_{b l}=0.6$ and base station density $\lambda_{b s}=0.3$.

4.8 The SNR outage probability at threshold $\beta=10 \mathrm{~dB}$ with respect to $\lambda_{b l}$ when $N=1,2$, with and without considering blockage correlation at fixed values of blockage density $\lambda_{b s}=0.3$ and blockage width $W=0.8$. . . . . . . . . . . . 56

4.9 Map of WVU downtown campus. The red-highlighted buildings are the blockages, and the base stations and user are randomly placed over the region. . 58

4.10 The distribution of SNR over 1000 network realizations when $N=1,2$ using diversity combining, plotted using the real data model and the analytical model. 58

4.11 The distribution of SINR over 1000 network realizations using diversity combining for different values of number of interfering transmitters. The curves are computed when $N=1,2$, with and without considering blockage correlation, at fixed values of $\lambda_{b s}=0.3, \lambda_{b l}=0.6$, and $W=0.6 . . . . . .61$

4.12 The outage probability of SINR at threshold $\beta=15 \mathrm{~dB}$ versus the number of interfering transmitters $(M)$, when $N=1,2$, with and without considering blockage correlation, at fixed values of $\lambda_{b s}=0.8$ and $W=0.6 . \quad$. . . . . . . 62

4.13 The outage probability of SINR at threshold $\beta=10 \mathrm{~dB}$ versus the macrodiveristy order $(N)$, when $M=1,3,10$, at fixed values of $\lambda_{b s}=0.8$ and $W=0.6 .63$

5.1 Example of 3D Urban aerial network consisting of UAVs, user vehicles, and buildings. Green solid lines indicate LoS links while red dashed lines indicate non-LoS. A shadow is shown directly below each UAV to identify its projection onto the ground plane. Street widths are exaggerated relative to building widths.

5.2 Coordinates associated with the $i^{\text {th }} \mathrm{UAV}$, including its location $X_{i}$ as projected onto the ground, the 2D distance $d_{i}$ from the vehicle to the projection, the azimuth angle $\phi_{i}$ relative to the positive $X$ axis, the height of UAV $h_{\mathrm{UAV}}$, the height of the vehicle antenna $h_{\mathrm{V}}$, and the 3D distance $r_{i} . \ldots$. . . . . . . 68

5.3 The connectivity distribution for urban and suburban deployments, considering two possible vehicle locations (at intersections or outside intersections) and the average across both locations. UAV density, UAV height, and maximum transmission range are fixed at $\lambda_{\mathrm{UAV}}=20 / \mathrm{km}^{2}, h_{\mathrm{UAV}}=100 \mathrm{~m}$, and $r_{\max }=250 \mathrm{~m}$. . . . . . . . . . . . . . . . . . . . . 73

5.4 The connectivity distribution when $r_{\max }=200 \mathrm{~m}$ and $300 \mathrm{~m}$ for dense urban, urban, and suburban deployments. UAV density and UAV height are fixed at values of $\lambda_{\mathrm{UAV}}=20 / \mathrm{km}^{2}, h_{\mathrm{UAV}}=100 \mathrm{~m} . \quad \ldots \ldots \ldots \ldots$

5.5 The outage probability at threshold $\gamma_{\text {th }}=0.8$ with respect to $h_{\text {UAV }}$ when $\lambda_{\text {UAV }}=10,20,30 / \mathrm{km}^{2}$. The deployment is urban and the maximum transmission range is fixed at $r_{\max }=250 \mathrm{~m}$. . . . . . . . . . . . . . . 76

5.6 The outage probability at threshold $\gamma_{\text {th }}=0.8$ with respect to $h_{\text {UAV }}$ for the three city types. UAV density and maximum transmission range are fixed at values of $\lambda_{\mathrm{UAV}}=20 / \mathrm{km}^{2}$ and $r_{\max }=250 \mathrm{~m} . \ldots \ldots \ldots$. . . . . . . . 77 
5.7 Contour plot showing the outage probability as a function of both UAV density $\lambda_{\text {UAV }}$ and UAV height $h_{\text {UAV }}$. Contours are spaced in increments of 0.1 , with 0.1 being the topmost curve. . . . . . . . . . . . . . . . . . 78

5.8 3D Contour plot showing the outage probability as a function of both UAV

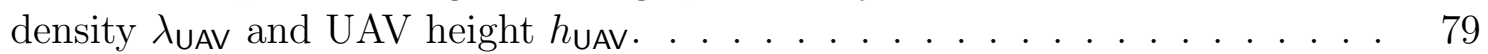

5.9 The optimal UAV height with respect to $\lambda_{\text {UAV }}$ for dense urban, urban, and suburban deployments. . . . . . . . . . . . . . . . . . . . . . . 79

5.10 Outage probability achieved when the optimal UAV height is used in dense urban, urban, and suburban deployments. . . . . . . . . . . . . . . . . 80 


\title{
List of Abbreviations
}

\author{
AWGN additive white Gaussian noise \\ BPP binomial point process \\ BS base station
}

CCDF complementary cumulative distribution function

CDF cumulative distribution function

D2D device-to-device

FSO free-space optical

LOS line-of-sight

MAC media access control

MIMO multiple-input and multiple-output

mmWave millimeter-wave

MPLP Manhattan Poisson line process

NLOS non line-of-sight

PDF probability density function

PPP Poisson point process

PMF probability mass function

SINR signal-to-interference-and-noise ratio 
SNR signal-to-noise ratio

UAV unmanned aerial vehicle

UE user equipment

WPAN wireless personal area networks 


\section{Chapter 1}

\section{Introduction}

\subsection{Motivation and Challenges}

In this section, several recent approaches used to achieve high data rates in wireless networks are outlined. These approaches involve moving to higher frequency bands that have significant amounts of bandwidth and good signal propagation features, and using antenna diversity techniques to increase spectral efficiency. This is followed by a discussion of the motivation for this research.

The most significant motivations for using millimeter-wave (mmwave) bands are The large amount of bandwidth available in mmWave frequency bands, good isolation, and better co-existence due to directional antennas. The global bandwidth shortage facing wireless carriers has inspired the exploration of the underutilized mmWave frequency spectrum for future broadband cellular communication networks [1]. Even with the advances of 4G LTE, the network is running out of bandwidth. The solution, as seen by $5 \mathrm{G}$ wireless network developers, is to add more bandwidth by using frequency spectrum in the millimeter-wave frequency range [2,3]. With hundreds of megaHertz of bandwidth available, 5G wireless networks will be capable of almost zero-latency communications at extremely high data speeds [4]. As shown in Fig. 1.1, the amount of bandwidth available at mmWave frequencies is enormous compared to the amount of frequency spectrum used by $4 \mathrm{G}$ and previous wireless network technologies.

At mmWave frequencies, signals are prone to blocking by objects intersecting the 

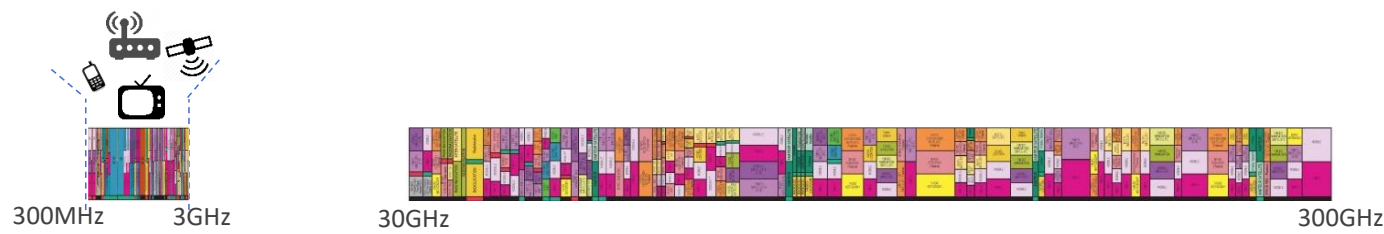

Figure 1.1: millimeter wave spectrum is the band of spectrum between $30 \mathrm{GHz}$ and 300 $\mathrm{GHz}$.
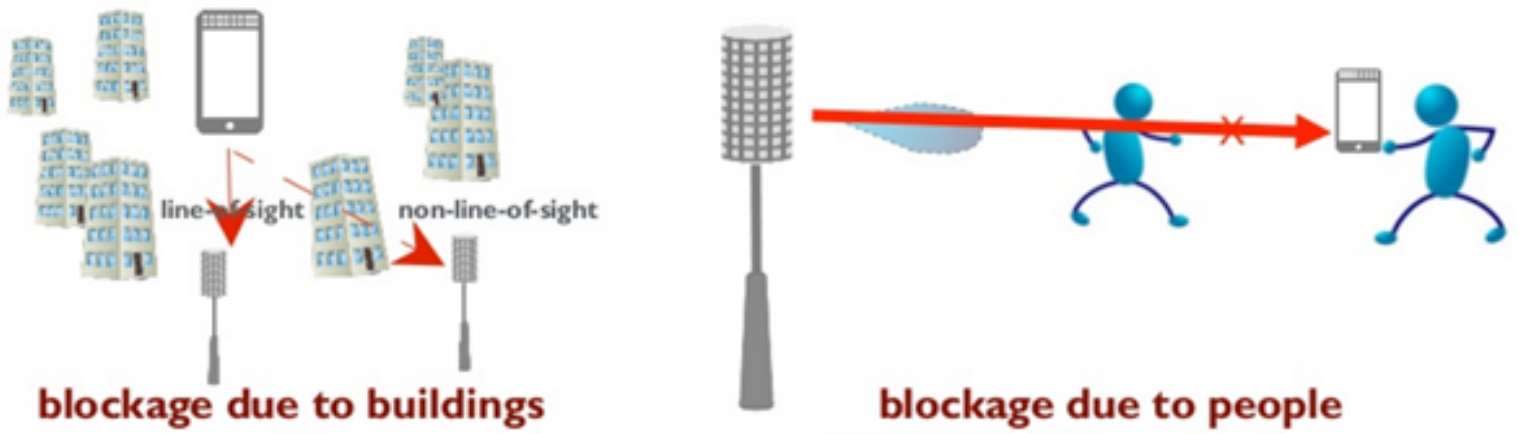

Figure 1.2: Example of blocking scenarios.

propagation paths including human bodies as shown in Fig. 1.2, Blocking makes it especially difficult to provide universal coverage with a cellular infrastructure. For instance, blockage by walls provides isolation between indoor and outdoor environments, making it difficult for an outdoor base station to provide coverage indoors [5]. Transmission losses at these frequencies also occur when signals travels through the atmosphere are absorbed by molecules of oxygen, water, and other gaseous atmospheric constituents [6]. While the path loss could be high, it can be compensated through the use of highly directional antennas, which is the next challenge after blocking because it is a requirement for any mmWave network to help with the path loss and also to isolate interference.

The mmWave band is characterized by high attenuation, which is both a blessing and a curse [7]. On the one hand, the desired signal is highly attenuated, and to overcome the attenuation, high gain directional antennas are required. However, due to the small wavelength, compact multi-element antenna arrays are feasible, even on a compact user terminal. On the other hand, interference tends to also be highly attenuated, and thus the band is characterized as having reasonable interference isolation [8].

MmWave has emerged as a promising solution for wireless personal-area networks (WPAN) 
and as an enabler for emerging applications such as high-resolution untethered virtual reality, augmented reality, and mixed reality [9 11]. The performance of mmWave systems can be characterized by the outage probability, or equivalently, by the cumulative distribution function (CDF) of the signal-to-interference and noise ratio (SINR). Alternatively, the performance can be characterized by the coverage probability, which is the complimentary CDF of the SINR, or the rate distribution, which can be found by using information theory to link the SINR to the achievable rate. Prior work has considered the SINR distribution of mmWave personal networks 12 14. Such work assumes that the blockages are drawn from a point process (or, more specifically, that the centers of the blockages are drawn from a point process and each blockage is characterized by either a constant or random width). Meanwhile, the interferers are either in fixed locations or their locations are also drawn from a point process. A universal assumption in this prior art is that the blocking is independent; i.e., each interferer is blocked independently from the other interferers.

In actual networks, blocking might be correlated. This is particularly true when two interferers are close to each other, or more generally when two interferers have a similar angle to the reference receiver. In this case, when one interferer is blocked, there is a significant probability that the other interferer is also blocked. However, correlated blocking can arise even when interferers are not close. Take, for instance, an extreme case where there is just one blockage in the environment and two interferers located far apart from each other. If the first interferer is blocked, then the second one cannot be blocked, giving rise to a negative correlation.

As blocking has a major influence on the distribution of the interference, it must be carefully taken into account. Independent blocking is a crude approximation that fails to accurately capture the true environment, especially when the interferers are closely spaced or when there are few sources of blocking. We note that blocking can be correlated even when the sources of blockage are placed independently according to a point process. The issue of blockage correlation was recently considered in [15, 16], but it was in the context of a localization application where the goal was to ensure that a minimum number of positioning transmitters were visible by the receiver. As such, it was only concerned with the number of unblocked transmissions rather than the distribution of the received aggregate signal (i.e., 
the interference power).

\subsection{Summary of Research Accomplishments}

Millimeter-wave systems are characterized by the use of highly directional antennas and the presence of blockages, which significantly alter the path-loss and small-scale fading parameters. The received power of each interferer depends on the direction it points and whether it is line-of-sight (LOS), non-LOS (i.e., partially blocked), or completely blocked [3]. While interferers that are sufficiently far away will almost certainly be completely blocked, a finite number of interferers in close proximity will be subject to random partial blockages. Previous attempts to characterize mmWave networks have made the simplifying assumption that all interferers within some radius, called the LOS ball, are unblocked, while interferers beyond that radius are non-LOS. However, compared to simulation results, the LOS ball assumption tends to overestimate outage.

This dissertation involves the development of an approach to analyse the SINR performance of mmWave wireless network. First, the exact outage probability is found for a finite network with interferers in fixed locations. Then, the spatially averaged outage probability is found by averaging over the interferer locations. While the focus is on device-to-device networks, the analysis is general enough to find applications outside of the present mmWave framework. The impact of user density, the directivity and array gains of the antennas used at the transceivers can be studied using this model.

Moreover, The dissertation investigates and models mmWave networks when considering corelated blocking. Closed form expressions for the blockage correlation coefficient and the distribution of the SINR are provided for the case of two dominant interferers and a fixed number of blockages drawn from a binomial point process. Finally, the effects of antenna directivity and the spatial randomness of the interferers are taken into account, resulting in SINR curves that fully account for correlated blocking, which are compared against curves that neglect correlation. The results provide insight into the validity of the commonly held assumption of independent blocking and the improved accuracy that can be obtained when the blocking correlation is taken into account. 
Macrodiversity is identified as an important strategy for mitigating blocking, as with macrodiversity, the user will attempt to connect with two or more base stations. Diversity is achieved because if the closest base station is blocked, then the next base station might still be unblocked. However, since it is possible for a single blockage to simultaneously block the paths to two base stations, the issue of correlated blocking must be taken into account by the analysis. Our analysis characterizes the macrodiverity gain in the presence of correlated random blocking and interference. To do so, we develop a framework to determine distributions for the LOS probability, Signal to Noise Ratio (SNR), and SINR by taking into account correlated blocking. We validate our framework by comparing our analysis, which models blockages using a random point process, with an analysis that uses real-world data in the form of a map of a major university's campus wherein the actual buildings are the blockages. We consider a cellular uplink with both diversity combining and selection combining schemes, and compare the performance of the two schemes. We also study the impact of blockage size and blockage density along with the effect of co-channel interference arising from other cells. We show that the assumption of independent blocking can lead to an incorrect evaluation of macrodiversity gain, as the correlation tends to decrease macrodiversity gain.

The thesis also focuses specifically on communications in urban environments, which are especially challenging due to the presences of buildings which are very large blockages. To compensate for this extreme environment, we explore the use of a novel architecture wherein the base stations are situated on unmanned aerial vehicles (UAVs) that provide connectivity to ground assets such as vehicles located on city streets (background on UAV networks in Sec. 1.4). The UAVs are assumed to be randomly deployed at a fixed height according to a two-dimensional point process. Millimeter-wave (mmWave) frequencies are used to avail of large available bandwidths and spatial isolation due to beamforming. The key performance metric considered in this part of the dissertation is the connectivity probability, which is the probability of an unblocked LoS path to at least one UAV within some maximum transmission distance. By leveraging tools from stochastic geometry, the connectivity probability is characterized as a function of the city type (e.g., urban, dense urban, suburban), density of UAVs (average number of UAVs per square km), 


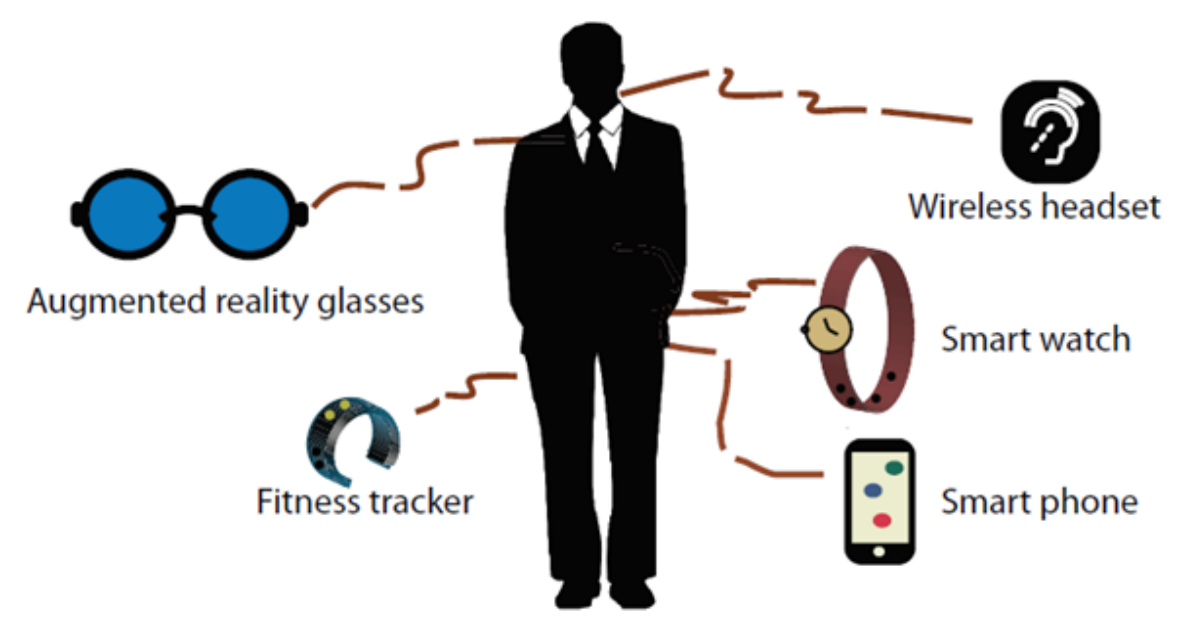

Figure 1.3: Several mobile wearable devices attached around a user's body a device to device network.

and height of the UAVs. The city streets are modeled as a Manhattan Poisson Line Process (MPLP) and the building heights are randomly distributed. The analysis first finds the connectivity probability conditioned on a particular network realization (location of the UAVs) and then removes the conditioning to uncover the distribution of the connectivity; i.e., the fraction of network realizations that will fail to meet an outage threshold. While related work has applied an MPLP to networks with a single UAV, the contributions of this part of the dissertation are that it extends that work by (1) considering networks of multiple UAVs, (2) characterizing the performance by a connectivity distribution, and (3) identifying the optimal altitude for the UAVs.

\subsection{Millimeter Wave: Device to Device Networks}

From a wireless communications perspective wearable communication networks are the next frontier for device-to-device (D2D) communication. Fig. 1.3 shows an example of deviceto-device (D2D) communication. Wearable networks connect different devices in and around the human body including low-rate devices like pedometers and high-rate devices like augmented or mixed-reality glasses. With the availability of newer commercial products, it seems feasible that many people will soon have multiple wearable devices [12].

Such a wearable network around an individual may need to operate effectively in the 
presence of interference from other users' wearable networks. This is problematic for applications that require Gbps throughput like virtual reality or augmented displays. The millimeter wave (mmWave) band contains a wide range of carrier frequencies capable of supporting short range high-rate wireless connectivity. Standards like Wireless HD and IEEE 802.11ad have already made mmWave-based commercial products a reality [7]. Wearable networks might use these standards or might use device-to device operating modes proposed for mmWave-based next generation $(5 \mathrm{G})$ cellular systems. Short-range mmWave communication systems usually focus on high-speed wireless connectivity to replace cable connections [3].

\subsection{Millimeter Wave: UAV to Ground Networks}

Aerial base stations, which are also known as Unmanned Aerial Vehicle Base Stations (UAV-BSs) have emerged in recent years as a viable candidate for mmwave-based networks, due to their flexible relocation, rapid deployment, and higher chances of experiencing Lineof-Sight (LoS) propagation path features have been perceived as promising opportunities to provide service in currently difficult to address service provisioning scenarios like short duration extremely crowded environments $[17,18$.

UAVs can be used to enhance existing ground base stations when there is a high demand for communication services. For example, UAVs can act as an additional base station for sports events etc. As shown in Fig. 1.4. UAVs can provide coverage for assets on the ground (i.e., vehicles) and provide services such as surveillance and real-time video transfer. Such applications are important for public safety, disaster recovery, and military operations. On the battlefield, UAVs can serve as drone base stations (BSs) in the air, to provide coverage in cases where ground BS do not exist or have been damaged [19, 20]. However, with the help of new technologies (e.g., tethered UAVs), UAVs have longer flight times that no longer limit aerial networks to temporary scenarios. For instance, UAVs are now candidates for providing cellular coverage, especially for challenging urban environments 21].

At mmWave frequencies, blocking by walls provides isolation between indoor and outdoor environments, making it difficult for an outdoor base station to provide coverage indoors 5 , 


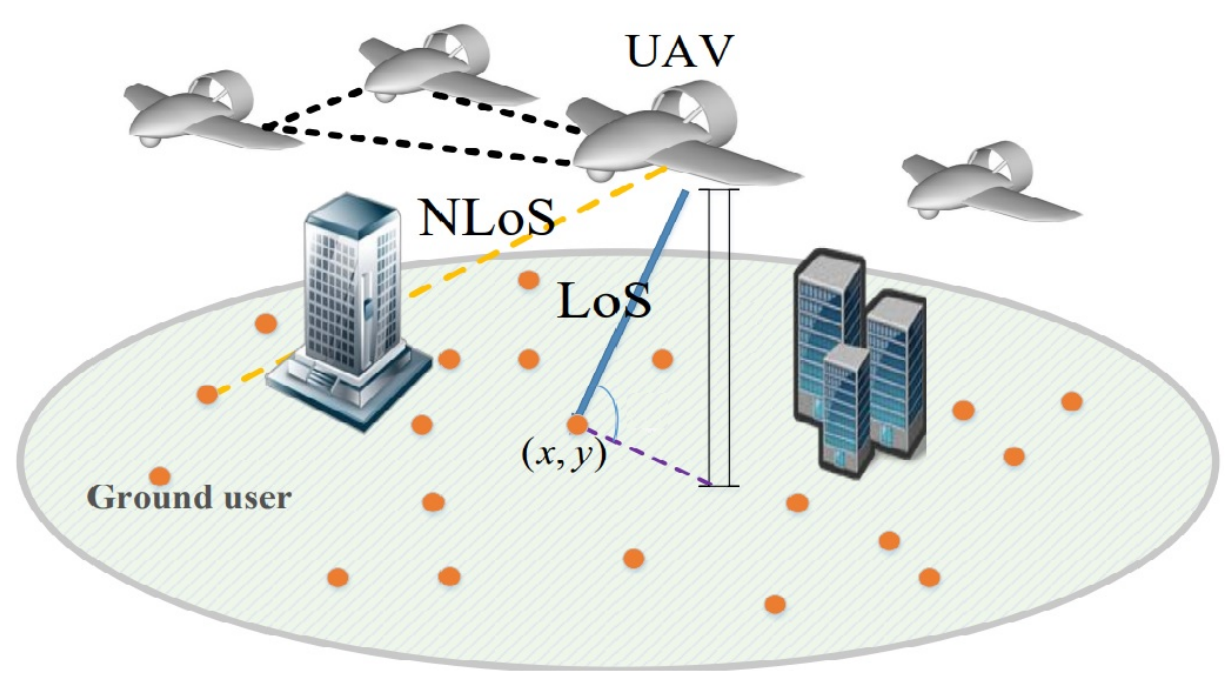

Figure 1.4: Example of UAV to ground communications

7,22. Outdoor networks in urban environments are challenging to design due to the presence of a high density of buildings, which serve as sources of blockage [23]. Implementing such networks using aerial networks is a good solution because the UAVs that serve as base stations may be quickly repositioned to overcome outages due to blocking, and they may be deployed at a sufficiently high altitude to avoid blocking by lower buildings.

When considering UAV-based networks, it is necessary to extend the model to three dimensions. In a city environment, the heights of buildings is a critical factor, because not all buildings between a ground-based user and a UAV will necessarily be tall enough to block the LoS path. To characterize a city, the locations of the buildings and their heights may be modeled through the tool of stochastic geometry.

\subsection{Stochastic Geometry: A Tool for Modern Net- works}

Stochastic geometry provides a mathematical approach for modeling wireless networks 24, 25 and is an effective methodology to study wireless systems in general, and mmWave systems in particular. The tools of stochastic geometry to analyze the outage, coverage, and rate of wireless networks 26$]$. With stochastic geometry, the locations of the user devices 
and blockages are assumed to be drawn from an appropriate point process. Stochastic geometry has been applied to mmWave cellular systems in 27 30 and mmWave D2D systems in 12,13$]$.

With stochastic geometry, the transceiver nodes in the network are modeled as randomly located in the 2-D plane to form a point process of known intensity, and the distribution of the interferers as seen by a typical user is analyzed as in [12. Stochastic geometry allows the derivation of analytic expressions for average performance metrics like the SINR, spectral efficiency, and the sum throughput for infinite networks [31].

In the context of mmWave-based cellular systems, [27] used results from stochastic geometry to characterize network coverage and capacity. The important propagation features of the mmWave signal, especially signal blockage due to buildings and human user body were modeled in [13]. In [5], a distance-dependent blockage model was first derived using results from random shape theory, and was then used to derive analytic expressions for SINR coverage. An important assumption for these derivations was that the sources of signal blockages (buildings) and the communication devices are drawn from independent Poisson Point Processes (PPP). For dense indoor operations using mmWave, human bodies of the users are the main source of blockages and can result in 30-60 dB of attenuation for mmWave signals [13. The users that carry potentially interfering devices, hence, also potentially block the interference from other users. This is a key difference compared to outdoor cellular based mmWave systems where independent spatial distribution assumption for blockages and interferers is easier to justify. Another point of difference between outdoor cellular systems and indoor mmWave systems is the spatial extent and number of transceiver devices considered in the analysis. While an infinite region of operation and infinite number of users could be justified in a cellular setting, system models for indoor operation have to consider finite geometry and number of users [12]. This provided valuable insight into the nature of surface reflections in the indoor mmWave setup. While it was assumed that the reflections from the ceilings were never blocked and the selfbody human blockage was characterized, 32 does not consider directional antennas at the devices. While [13] reports closed-form analytic expressions for spatially averaged system performance.

The tool of stochastic geometry has been extensively used to study interference in large 
wireless networks [29]. Prior work on mmWave-based networks has also used the results from stochastic geometry to analyze coverage and rate 27] while modeling the directionality of antennas and the effect of blockages [13. For analytical tractability, most work assumes an infinite number of mobile devices spread over an infinite area 27. These assumptions allow the analytical expressions related to the spatial average of the system performance to be simplified through application of Campbell's theorem [29]. Analysis of the outage probability conditioned on the network geometry in ad hoc networks with a finite spatial extent and number of interferers was performed in [33].

\subsection{Organization}

In the sequel, the research work is discussed in more detail. In Chapter 2, an outline of the completed work related to modeling device to device networks for fixed and random locations is discussed. The impact of correlated blocking in mmWave networks is presented in Chapter 3. Chapter 4, identifies and accurately analyzes macrodiversity as a solution for blockage in presence of correlated blocking and interference. Chapter 5 identifies the connectivity probability and provides an optimization of UAV to ground networks in urban environments. Chapter 6 provides a summary of the dissertation and the future work. 


\section{Chapter 2}

\section{Accurately Accounting for Random Blockage in Device-to-Device Millimeter-Wave Networks}

\section{$2.1 \quad$ Introduction}

Millimeter-wave (mmWave) has emerged in recent years as a viable candidate for both device-to-device (D2D) communications as well as infrastructure-based (i.e., cellular) systems $3,7,12,13,27,30,34$. At mmWave frequencies, signals are prone to blocking by objects intersecting the propagation paths. While the path loss could be high, it can be compensated through the use of highly directional antennas, which also helps to isolate interference. Blocking can significantly impact the distribution of the small-scale fading (i.e., resulting in a non line-of-sight state) and if severe enough, cause the signal to be lost completely (i.e., resulting in an outage state) [3]. The power of each received signal, whether it be a desired signal or an interfering signal, is thus highly dependent on the relative orientations of the transmit and receive antennas and the presence of objects blocking the paths. Any meaningful analysis of mmWave systems must therefore account for antenna orientation and blockage, and typically these are modeled as appropriate random processes.

An effective methodology to study wireless systems in general, and mmWave systems in particular, is to embrace the tools of stochastic geometry to analyze the outage, coverage, 
and rate of wireless networks $[26]$. With stochastic geometry, the locations of the interferers and blockages are assumed to be drawn from an appropriate point process. Stochastic geometry has been applied to mmWave cellular systems in 27 30 and mmWave D2D systems in 12,13 . Stochastic geometry provides a tractable means for finding the outage probability, coverage, and rate when the interferers and blockages are drawn from random point processes.

A survey of mathematical models and analytical techniques is provided in [30 with a section devoted to blockage models. Random shape theory, which is an offshoot of stochastic geometry, is applied in [5] to carefully consider blockage effects. When blocking is modeled as a random process, the probability that a link is line-of-sight (LOS), i.e., not blocked, is an exponentially decaying function of link distance. The distance-dependent blocking probability causes significant challenges to the application of stochastic geometry. This challenge can be overcome by making a simplifying assumption that all interferers within some radius, called the $L O S$ ball, are unblocked, while interferers beyond that radius are nonLOS. The LOS ball assumption has been applied to mmWave cellular in [27,28] and D2D in [13]. Meanwhile, a two-ball approximation was applied to mmWave multi-tier cellular systems in 29]. While it aids tractability, the LOS ball assumption causes a non-negligible loss in accuracy. For instance, in [13, the LOS ball approximation caused the distribution of coverage to be underestimated by a few decibels.

In this chapter, we propose an analytical framework for mmWave networks that explicitly accounts for the blockage probabilities, thereby dispensing with the need for a LOS ball. The key to the analysis is to break it into two steps. In the first step, the interferers are placed in fixed locations and the outage probability found conditioned on the interferers' locations. Each interferer is characterized by a fading distribution that can take on a plurality of states, depending on the random orientation of the antennas and random blockage probabilities. In the second step, the distribution of the outage is found by taking the spatial average of the conditional outage probability over the distribution of the interferer locations. Simulation results confirm the accuracy of the strategy and demonstrate its superiority over the LOS ball assumption.

The focus of the chapter is on D2D networks, whereby the interferers transmit with a common power in a uniformly distributed direction. However, the analysis could be extended 
to the more complicated case of a cellular network, where each interferer's transmit power and direction are correlated with the location of its serving base station. The analysis is generic enough that it could find applications outside of mmWave, such as in the area of frequency hopping [33].

The remainder of the chapter is organized as follows. Section 2.2 gives a system model and provides a general problem formulation. Section 2.3 derives an expression for the outage probability conditioned on the location of the interferers, and Section 2.4 applies it to a D2D mmWave network. Section 2.5 provides an approach for obtaining the spatially averaged outage probability. Finally, the chapter concludes in Section 2.6 .

\subsection{System Model}

Consider a wireless network with a reference receiver, a reference transmitter, and $K$ interferers located within some area $\mathcal{A}$. While the network itself may have an infinite extent and therefore an infinite number of interferers, we assume that very distant interferers are fully attenuated and therefore do not contribute directly to the interference power (though they could contribute to the noise floor). Only a finite number $(K)$ of interferers are close enough to contribute to the interference power, though the contribution of each will depend critically on whether or not its signal is LOS or non-LOS. Moreover, the number of interferers $K$ could itself be random. For instance, if the interferers are drawn from a Poisson point process (PPP), then the number of interferers in $\mathcal{A}$ will be a Poisson variable.

Define the variable $S$ to represent the signal-to-interference and noise ratio (SINR) at the reference receiver. Our goal is to find an expression for the outage probability as a function of an SINR threshold $\beta$, which is the cumulative distribution function (CDF) of $S$; i.e, $F_{S}(\beta)$. The variable $S$ can be expressed as

$$
S=\frac{Y_{0}}{c+\sum_{i=1}^{K} Y_{i}} .
$$

where $c$ is a constant related to the noise power, $Y_{0}$ is the received power of the reference transmitter, and $\left\{Y_{i}\right\}, i \in\{1, \ldots, K\}$, are the received powers of the $K$ interferers. We assume that $Y_{0}$ is a Gamma distributed random variable with a fixed shaping parameter $m_{0}$ and 
scale parameter $\eta_{0}$.

The value of $c$ is selected so that the signal-to-noise ratio SNR is the mean value of $S$ when the interference is turned off; i.e.

$$
\mathrm{SNR}=\mathbb{E}\left[\frac{Y_{0}}{c}\right] \Longrightarrow c=\frac{\mathbb{E}\left[Y_{0}\right]}{\mathrm{SNR}}
$$

The other $Y_{i}, i \in\{1, \ldots, K\}$, each have a distribution that depends on a variety of factors including the distance to the interferer, the relative orientations of the transmit and receive antennas, the random transmission activity (e.g., use of an Aloha-like protocol), and the blockage process. We thus assume that each $Y_{i}, i \in\{1, \ldots, K\}$, is drawn from one of $J+1$ power distributions, each corresponding to a different state that encapsulates the blockage and directivity conditions. This is done by drawing a discrete random variable $a_{i} \in\{0,1, \ldots, J\}$, which indicates the chosen power distribution. Let $p_{i, j}$ represent the probability that $a_{i}=j$ for $i \in\{1,2, \ldots, K\}$ and $j \in\{0,1, \ldots, J\}$. The probabilities $\left\{p_{i, j}\right\}$ could depend on the location $X_{i}$ of the $i^{\text {th }}$ interferer. For instance, if a random blockage model is assumed, then the probabilities associated with blockage states will be functions of the distance to the interferer.

Let $a_{i}=0$ represent the specific case that the interferer is turned off (or not using the same resource as the reference transmitter). It follows that $Y_{i}=0$ when $a_{i}=0$, and thus the corresponding power distribution has probability density function (PDF) $f_{Y_{i}}\left(y \mid a_{i}=\right.$ $0)=\delta(y)$. Otherwise, when $a_{i}>0$, we assume that the variable is Gamma distributed. We define two functions: $m\left(a_{i}\right)$ which describes the shaping parameter associated with distribution $a_{i}$ and $\eta\left(a_{i}\right)$ which describes the scaling factor of distribution $a_{i}$. The mean of $Y_{i}$ is $\mathbb{E}\left[Y_{i}\right]=m\left(a_{i}\right) / \eta\left(a_{i}\right)$. To make the notation more compact, we will use double subscripts for $m(\cdot)$ and $\eta(\cdot)$, so that $m\left(a_{i}=j\right)=m_{i, j}$ and $\eta\left(a_{i}=j\right)=\eta_{i, j}$. Due to path-loss and the orientation of the reference receiver's antenna, these functions generally depend on the location of the $i^{t h}$ interferer, which we denote $X_{i}$. It follows that the PDF when $a_{i}=j$ is

$$
f_{Y_{i}}\left(y \mid a_{i}=j\right)=\frac{\eta_{i, j}^{m_{i, j}}}{\Gamma\left(m_{i, j}\right)} y^{m_{i, j}-1} e^{-\eta_{i, j} y} u(y)
$$

where $u(y)$ is the unit step function and $\Gamma(\cdot)$ is the Gamma function. 


\subsection{Conditional Outage Probability}

Assume that the interferers are in fixed locations. From the theorem on total probability, the PDF of $Y_{i}, i \in\{1, \ldots, K\}$, can simply be found from the weighted sum of the conditional probabilities.

$$
\begin{aligned}
f_{Y_{i}}(y) & =\sum_{j=0}^{J} p_{i, j} f_{Y_{i}}\left(y \mid a_{i}=j\right) \\
& =p_{i, 0} \delta(y)+\sum_{j=1}^{J} \frac{p_{i, j} \eta_{i, j}^{m_{i, j}}}{\Gamma\left(m_{i, j}\right)} y^{m_{i, j}-1} e^{-\eta_{i, j} y} u(y) .
\end{aligned}
$$

The CDF of $S$ can then be found as

$$
\begin{gathered}
F_{S}(s)=P[S \leq s]=P\left[Y_{0} \leq s\left(c+\sum_{i=1}^{K} Y_{i}\right)\right] \\
=\int \ldots \iint_{0}^{s\left(c+\sum_{i=1}^{K} y_{i}\right)} f_{Y_{0}}\left(y_{0}\right) d y_{0} f_{\boldsymbol{Y}}(\boldsymbol{y}) d \boldsymbol{y},
\end{gathered}
$$

where $f_{\boldsymbol{Y}}(\boldsymbol{y})$ is the joint PDF of $\mathbf{Y}=\left(Y_{1}, Y_{2}, \ldots, Y_{K}\right)$, and the inner integral of $f_{Y_{0}}\left(y_{0}\right)$ is the $\mathrm{CDF}$ of $Y_{0}$ evaluated at $s\left(c+\sum_{i=1}^{K} y_{i}\right)$. Substituting this CDF into 2.4 leads to

$F_{S}(s)=1-e^{-\eta_{0} s c} \sum_{l=0}^{m_{0}-1} \frac{1}{l !}\left(\eta_{0} s c\right)^{l} \int \ldots \int e^{-\eta_{0} s \sum_{i=1}^{K} y_{i}}\left(1+\frac{\sum_{i=1}^{K} y_{i}}{c}\right)^{\ell} f_{\boldsymbol{Y}}(\boldsymbol{y}) d \boldsymbol{y}$.

Using the binomial theorem,

$$
\left(1+\frac{\sum_{i=1}^{K} y_{i}}{c}\right)^{\ell}=\sum_{t=0}^{\ell}\left(\begin{array}{l}
\ell \\
t
\end{array}\right) \frac{1}{c^{t}}\left(\sum_{i=1}^{K} y_{i}\right)^{t}
$$

and a multinomial expansion,

$$
\left(\sum_{i=1}^{K} y_{i}\right)^{t}=t ! \sum_{t_{i} \in \mathcal{T}_{t}} \prod_{i=1}^{K} \frac{y_{i}^{t_{i}}}{t_{i} !}
$$

where $\mathcal{T}_{t}$ the set of all nonnegative $t_{i}$ that sum to $t$. Substituting (2.6) and (2.7) into (2.5) yields

$$
F_{S}(s)=1-e^{-\eta_{0} s c} \sum_{\ell=0}^{m_{0}-1} \frac{1}{\ell !}\left(\eta_{0} s c\right)^{\ell} \sum_{t=0}^{\ell}\left(\begin{array}{l}
\ell \\
t
\end{array}\right) \frac{t !}{c^{t}} \sum_{t_{i} \in \mathcal{T}_{t}} \prod_{i=1}^{K} \frac{1}{t_{i} !} \int \ldots \int y_{i}^{t_{i}} e^{-\eta_{0} s y_{i}} f_{\boldsymbol{Y}}(\boldsymbol{y}) d \boldsymbol{y} .
$$


Since $Y_{1}, Y_{2}, \ldots$, and $Y_{K}$ are independent random variables, 2.8) can be rewritten as

$$
F_{S}(s)=1-e^{-\eta_{0} s c} \sum_{\ell=0}^{m_{0}-1} \frac{1}{\ell !}\left(\eta_{0} s c\right)^{\ell} \sum_{t=0}^{\ell}\left(\begin{array}{l}
\ell \\
t
\end{array}\right) \frac{t !}{c^{t}} \sum_{t_{i} \in \mathcal{T}_{t}} \prod_{i=1}^{K} \frac{1}{t_{i} !} \int_{0}^{\infty} y_{i}{ }^{t_{i}} e^{-\eta_{0} s y_{i}} f_{Y_{i}}\left(y_{i}\right) d y_{i}
$$

where the integral is

$$
\int_{0}^{\infty} y_{i}^{t_{i}} e^{-\eta_{0} s y_{i}} f_{Y_{i}}\left(y_{i}\right) d y_{i}=p_{i, 0} \delta\left(t_{i}\right)+\sum_{j=1}^{J} \frac{p_{i, j} \eta_{i, j}^{m_{i, j}}}{\Gamma\left(m_{i, j}\right)}\left(\eta_{0} s+\eta_{i, j}\right)^{-t_{i}-m_{i, j}} \Gamma\left(t_{i}+m_{i, j}\right) .
$$

Substituting 2.10) into 2.9) gives the expression

$$
\begin{aligned}
F_{S}(s)= & 1-e^{-\eta_{0} s c} \sum_{\ell=0}^{m_{0}-1} \frac{\left(\eta_{0} s c\right)^{\ell}}{\ell !} \sum_{n=0}^{\ell}\left(\begin{array}{l}
\ell \\
t
\end{array}\right) \frac{t !}{c^{t}} \\
& \times \sum_{t_{i} \in \mathcal{T}_{t}} \prod_{i=1}^{K}\left[p_{i, 0} \delta\left(t_{i}\right)+\sum_{j=1}^{J} \frac{p_{i, j} \eta_{i, j}^{m_{i, j}}}{\Gamma\left(m_{i, j}\right) t_{i} !}\left(\eta_{0} s+\eta_{i, j}\right)^{-t_{i}-m_{i, j}} \Gamma\left(t_{i}+m_{i, j}\right)\right]
\end{aligned}
$$

\subsection{Application to mmWave}

Consider the mmWave ad hoc network shown in Fig. 2.1. The reference receiver (represented by the red star) is located at the origin, while the $K$ interferers (represented by the blue dots) are located in an area $\mathcal{A}$, which here is assumed to be an annulus with inner radius $r_{\text {in }}$ and outer radius $r_{\text {out }}$. It is assumed that a MAC protocol (such as CSMA) prevents any interference closer than $r_{\text {in }}$ to the receiver, while the blockage is so severe at distance $r_{\text {out }}$ that signals beyond that distance are completely attenuated. Each interferer within $\mathcal{A}$ can either be unblocked, in which case its signal is LOS, or (partially) blocked, in which case its signal is non-LOS and highly (but not fully) attenuated.

The transmitter locations $X_{i}$ are represented by complex numbers, so that $X_{i}=R_{i} e^{j \phi_{i}}$, where $R_{i}$ denotes the distance from the $i^{\text {th }}$ transmitter to the receiver and $\phi_{i}$ is the angle from $X_{i}$ to the receiver. The reference transmitter (represented by the red dot) is located at a distance $R_{0}$ from the receiver, and in this example, $R_{0}=r_{\text {in }}$.

Assume that there are $K$ blockages in the network, and that each blockage is modeled by a disk of width $W$. We assume that the number of blockages is the same as the number of interferers because in an mmWave ad hoc network, a main source of blockage is human 


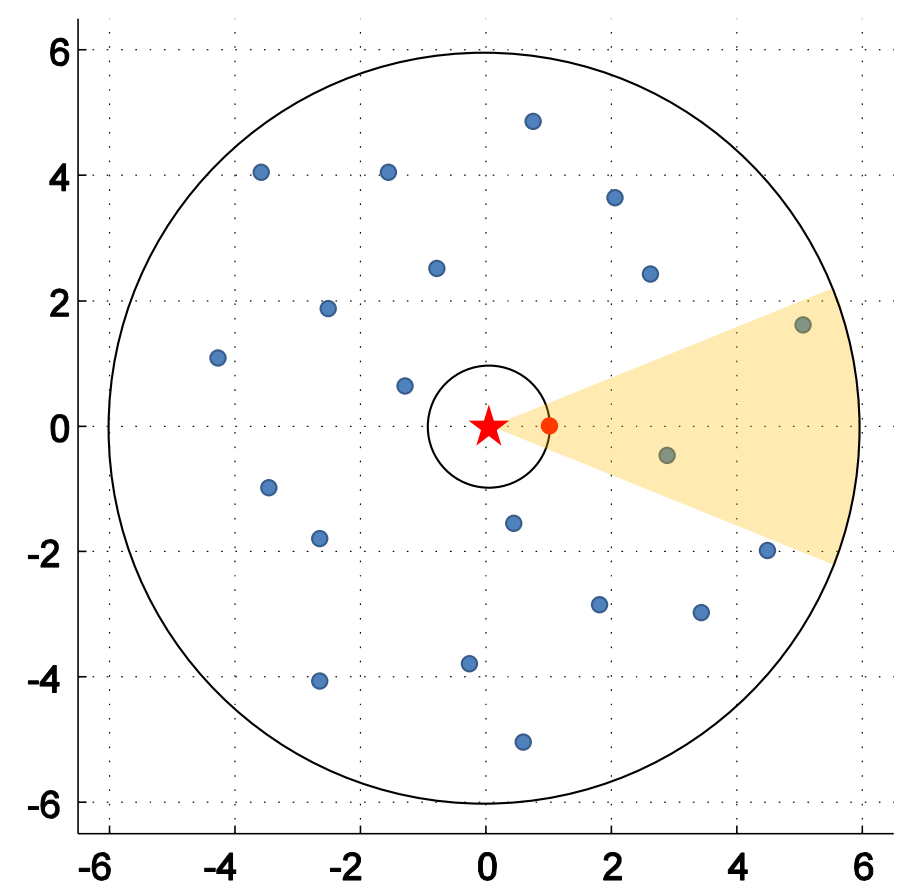

Figure 2.1: Network Topology. The $\mathrm{K}=20$ interferers are represented by the blue dots, the reference transmitter represented by the red dot, and the reference receiver represented by the red star. The yellow shaded area is the main lobe of the receiver's antenna.

bodies, and if we assume the interference is due to personal devices (e.g., wearables), then there will be approximately one interferer per person. Assuming that the blockages are independent and uniformly distributed over the annular region, the probability that an interferer at distance $r$ from the receiver is blocked by any of the $K$ blockages is given by $p_{b}(r)$. An equation and derivation for $p_{b}(r)$ is given in [13], and is incorporated herein by reference.

As in [13.27, we assume directional antennas that satisfy a sectorized model. In particular, the antenna gain is $G$ inside the (half-power) beamwidth $\theta$, and $g$ outside the beamwidth. The number of antenna elements is $N$ and the relationship between $N, G, g$, and $\theta$ is given by Table I in [13]. We use subscripts $t$ and $r$ to distinguish the parameters associated with the transmitter and receiver antennas, respectively. Thus, $N_{\mathrm{r}}$ is the number of elements of the receive antenna. The shaded area of Fig. 2.1 shows the main beam of the receive antenna. Assuming a random 2-D orientation for the interfering transmitters, the probability that an interferer points toward the receiver is $\frac{\theta_{t}}{2 \pi}$. 
We define $J=4$ transmission states corresponding to whether the interferer is or is not blocked and whether the interferer is pointing towards or away from the receiver. In particular, we let $a_{i}=\{1,3\}$ when the interferer is blocked and $a_{i}=\{2,4\}$ when it is not, and we let $a_{i}=\{1,2\}$ when the interferer is pointing towards the receiver and $a_{i}=\{3,4\}$ when it is pointing away. Moreover, we assume an Aloha-like medium access protocol, so that the probability that the interferer transmits is $p_{\mathrm{t}}$. Thus, the probability of state $a_{0}$, corresponding to a non-transmission state, is $\left(1-p_{\mathrm{t}}\right)$. It follows that the probabilities of the five states are:

$$
a_{i}=\left\{\begin{array}{lll}
0 & \text { with prob. }\left(1-p_{t}\right) \\
1 & \text { with prob. } & p_{b}\left(R_{i}\right) \frac{\theta_{t}}{2 \pi} p_{t} \\
2 & \text { with prob. } & \left(1-p_{b}\left(R_{i}\right)\right) \frac{\theta_{t}}{2 \pi} p_{t} \\
3 & \text { with prob. } & p_{b}\left(R_{i}\right)\left(1-\frac{\theta_{t}}{2 \pi}\right) p_{t} \\
4 & \text { with prob. } & \left(1-p_{b}\left(R_{i}\right)\right)\left(1-\frac{\theta_{t}}{2 \pi}\right) p_{t}
\end{array}\right.
$$

Each of the above $a_{i}$ implies specific shaping and scale parameters for the interferer's power distribution. In particular, the value of the shaping parameter $m_{i, j}$ depends on the blockage state. When the link is blocked, i.e. when $a_{i}=\{1,3\}$, the shaping parameter is $m_{i, j}=m_{\mathrm{N}}$; otherwise $m_{i, j}=m_{\mathrm{L}}$, where $m_{\mathrm{L}}$ and $m_{\mathrm{N}}$ are the LOS and non-LOS shaping parameters, respectively.

Moreover, the scaling parameter for the $i^{\text {th }}$ interferer depends on its distance $R_{i}$ as well as its state $a_{i}$, and each state could have associated with it a different antenna gain and path-loss exponent. The $\eta_{i, j}$ parameter is given by $\eta_{i, j}=m_{i, j} / \Omega_{i, j}$ where $\Omega_{i, j}$ is the average received power given by

$$
\Omega_{i, j}=g_{r}\left(\phi_{i}\right) g_{t}\left(a_{i}\right) R_{i}^{-\alpha_{j}}
$$

the receive antenna gain is

$$
g_{r}\left(\phi_{i}\right)= \begin{cases}G_{r} & \text { if }\left|\phi_{i}-\phi_{0}\right|<\frac{\theta_{r}}{2} \\ g_{r} & \text { otherwise }\end{cases}
$$


the transmit antenna gain is

$$
g_{t}\left(a_{i}\right)= \begin{cases}G_{t} & \text { for } a_{i} \in\{1,2\} \\ g_{t} & \text { for } a_{i} \in\{3,4\}\end{cases}
$$

and $\alpha_{j}=\alpha_{\mathrm{N}}$ if the link is blocked and $\alpha_{j}=\alpha_{L}$ if it is not.

We assume that the reference link is LOS; i.e., $m_{0}=m_{L}$. Because the reference transmitter and reference receiver point towards one another, $\eta_{0}=m_{0} / \Omega_{0}$ where

$$
\Omega_{0}=G_{r} G_{t} R_{0}^{-\alpha_{0}}
$$

and $\alpha_{0}=\alpha_{L}$.

Example \#1: We consider as an example a network of inner radius $r_{\text {in }}=1$, outer radius $r_{\text {out }}=6$, and $K=20$ interferers. The length of the reference link is $R_{0}=r_{\text {in }}=1$. The transmitters and receiver have $N_{\mathrm{t}}=N_{\mathrm{r}}=4$ antennas. The width of each blockage is $W=1$ and we assume that there are $K$ such blockages. The shape parameter (i.e., Nakagami-m factor) for LOS links is $m_{\mathrm{L}}=4$, while that of non-LOS links is $m_{\mathrm{N}}=1$ (i.e., Rayleigh fading). The path-loss exponent for LOS links is $\alpha_{\mathrm{L}}=2$, while that of non-LOS links is $\alpha_{\mathrm{N}}=4$. The probability that an interferer transmits is $p_{\mathrm{t}}=0.5$, and the signal-to-noise ratio is $\mathrm{SNR}=20$ dB.

Fig. 2.2 shows the outage probability for this example as a function of SINR threshold $\beta$ conditioned on the network realization shown in the left side of the figure. The outage probability is found two ways: By using 2.11, which accurately accounts for the blocking probability, and by using the LOS-ball approximation, which assumes all interferers within distance $R_{\text {Los }}$ are LOS and those beyond that distance are non-LOS [13]. Two values of $R_{\mathrm{LOS}}$ are used. The first, $R_{\mathrm{LOS}}=4.4$ is found by matching moments; i.e., by using criterion 1 of 27. The second, $R_{\mathrm{LOS}}=3.4$ is found by selecting the value of $R_{\mathrm{LOS}}$ that generates an outage probability curve that most closely matches (in a mean-square error sense) the curve found by the exact analysis. Note that finding $R_{\text {Los }}$ in this manner is not a sustainable solution because it requires that the exact probability be first found prior to finding the $R_{\text {Los }}$ that provides the best fit. Moreover, the best-fit value of $R_{\text {Los }}$ will change from one network realization to another. Hence, the purpose of the curve is to give insight into the best one 


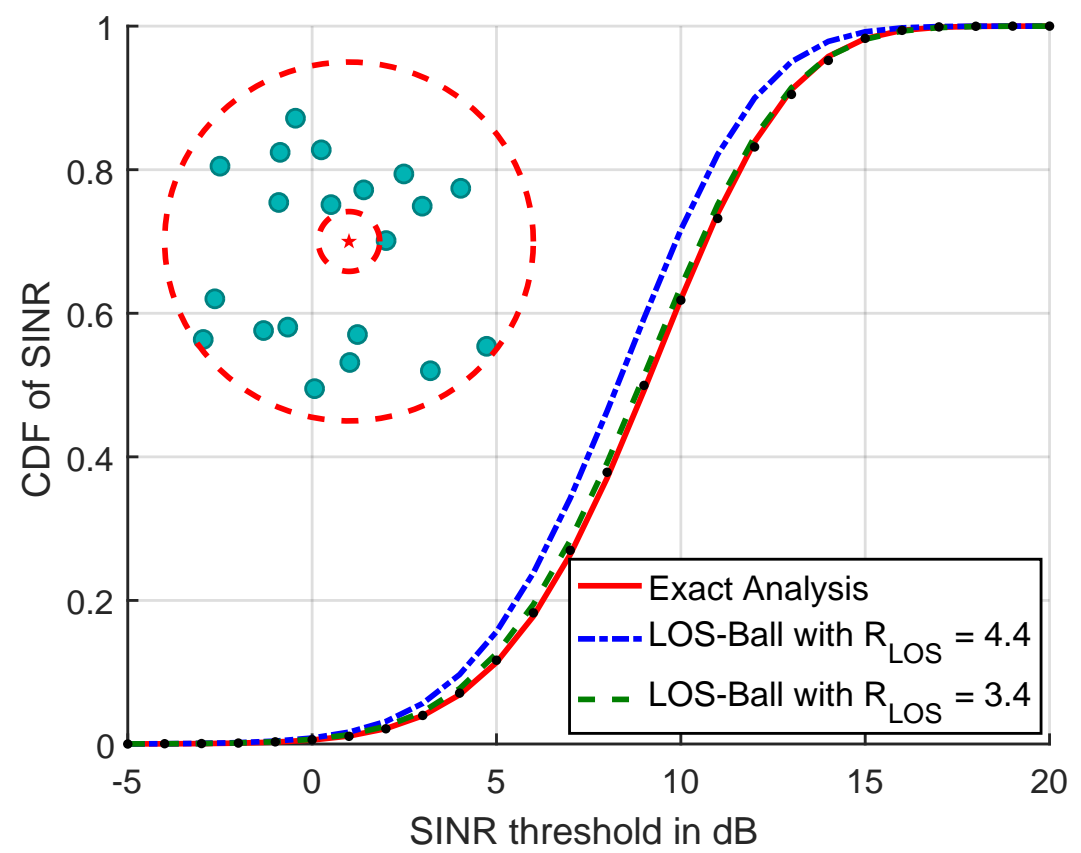

Figure 2.2: An example network in the upper left portion of the figure. The curves show the outage probability for this particular network at SNR $=20 \mathrm{~dB}$. The black dots represent simulation results. In addition to the exact outage probability found using the methods of this chapter, the outage probability using the LOS-ball assumption is shown with two values of $R_{\mathrm{LOS}}$.

can do when using the LOS ball assumption, even if an "optimal" value of $R_{\text {Los }}$ were to be used.

In addition, simulations were run to confirm the analysis, and are shown as dots on the figure. For each value of SNR, the simulation involved drawing 10,000 realizations of $S$, where each realization of $S$ required first drawing the necessary set of $a_{i}, i \in\{1, \ldots, L\}$, and then drawing the set of $Y_{i}, i \in\{0, . ., K\}$. Each dot shows the fraction of trials whose outage probability is less than the value on the ordinate. As can be seen, the proposed analytical technique provides close agreement. Moreover, the figure shows the superiority of the exact analysis compared to the LOS-ball assumption, especially when the value $R_{\text {Los }}=4.4$ is used. While $R_{\text {LOS }}=3.4$ provides a close agreement with the simulations, finding that optimal value of $R_{\text {LOS }}$ required the exact outage probability curves to first be found and thus its use does not simplify the overall analysis.

Fig. 2.4 shows the coverage probability which is found by taking the complementary 


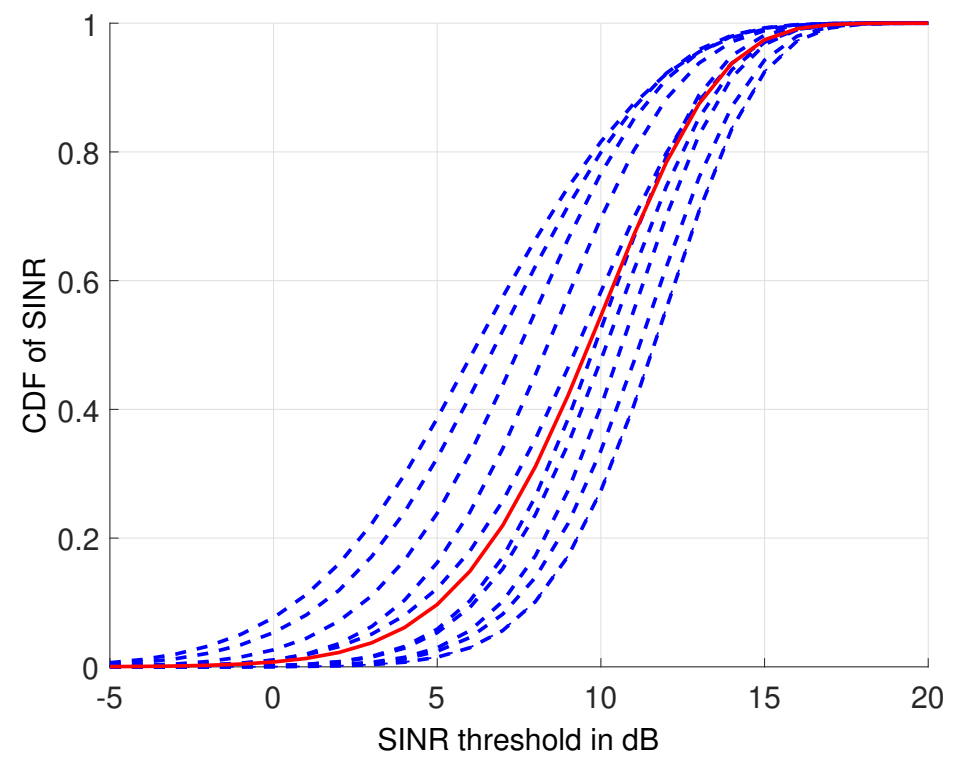

Figure 2.3: The outage probability conditioned on several network realization is plotted by dashed lines. The average outage probability over 100 network realization is plotted by the solid red line.

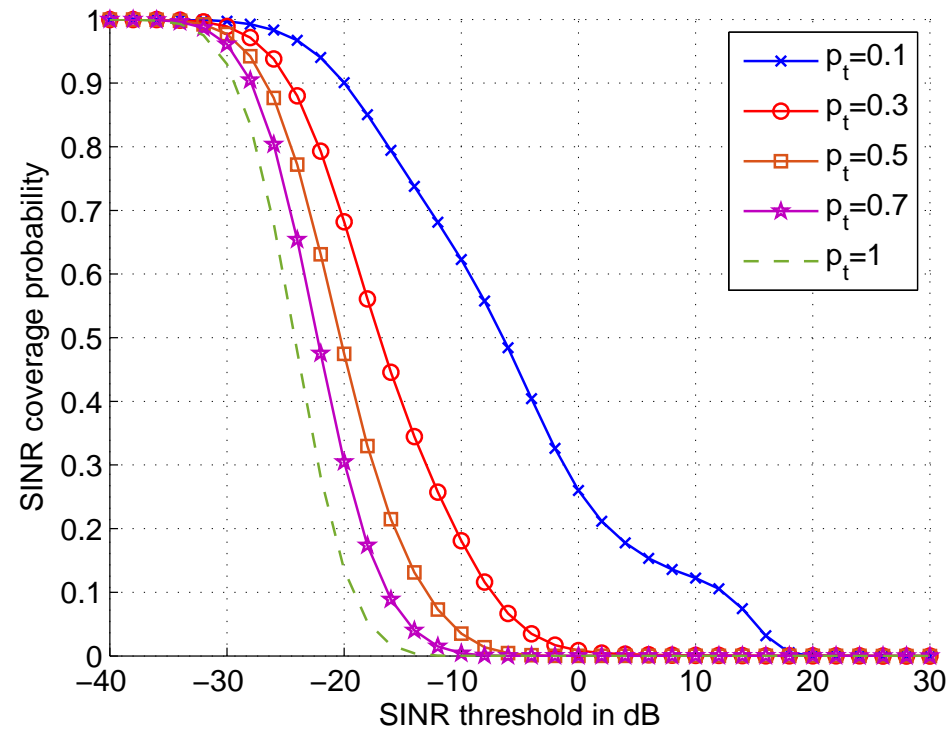

Figure 2.4: SINR coverage probability for different transmission probability $p_{\mathrm{t}}$ 


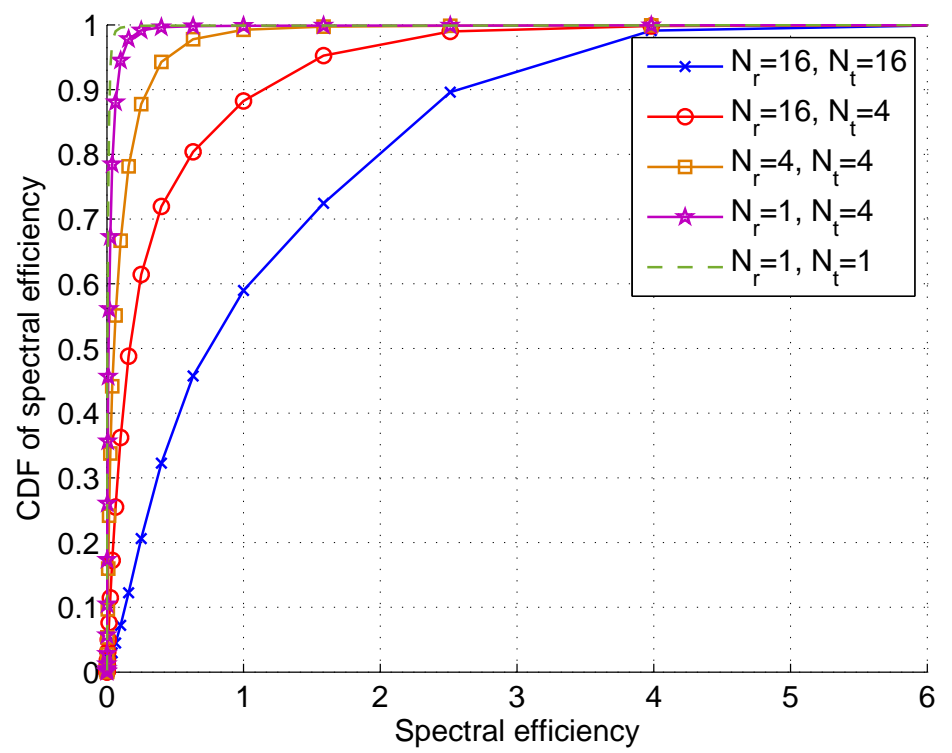

Figure 2.5: CDF of spectral efficiency for different number of antenna arrays at the transmitter and the receiver

CDF of SINR by using the CDF in 2.11. The coverage probability depends on the value of transmission probability $p_{\mathrm{t}}$ of the interferers. The higher value of $p_{\mathrm{t}}$ leads to lower SINR coverage probability.

Fig. 2.5 shows the CDF of spectral efficiency for different numbers of antenna elements for the transmitter and the receiver. Clearly, using more antenna elements result in significant improvement in the rate. This is because more antenna elements provide more directional transmission and provide higher gain at the reference link. Also the larger antenna elements reduces the beam width of the interferers which reduce the likelihood for an interferer to be pointing toward the receiver.

\subsection{Spatially Averaged Outage Probability}

The conditional outage probability depends significantly on the underlying network geometry. Fig. 2.3 shows the outage probabilities of 10 realizations of networks with $K=20$ interferers located in the annulus of inner radius $r_{\text {in }}=1$ and outer radius $r_{\text {out }}=6$. The curves were found using the analytical expression (2.11) for a SNR $=15 \mathrm{~dB}$. The figure illustrates that the outage probability can vary dramatically for different network realizations. 


$$
\begin{aligned}
E_{\phi_{i}} & {\left[Q_{t_{i}}^{\alpha_{j}}\left(\frac{g_{r}\left(\phi_{i}\right) g_{t}\left(a_{i}\right)}{T_{k+1}^{\alpha_{j}}}\right) T_{k+1}^{2}-Q_{t_{i}}^{\alpha_{j}}\left(\frac{g_{r}\left(\phi_{i}\right) g_{t}\left(a_{i}\right)}{T_{k}^{\alpha_{j}}}\right) T_{k}^{2}\right]=} \\
& \frac{\theta_{r}}{2 \pi}\left[Q_{t_{i}}^{\alpha_{j}}\left(\frac{G r g_{t}\left(a_{i}\right)}{T_{k+1}^{\alpha_{j}}}\right) T_{k+1}^{2}-Q_{t_{i}}^{\alpha_{j}}\left(\frac{G r g_{t}\left(a_{i}\right)}{T_{k}^{\alpha_{j}}}\right) T_{k}^{2}\right] \\
& +\left(1-\frac{\theta_{r}}{2 \pi}\right)\left[Q_{t_{i}}^{\alpha_{j}}\left(\frac{\operatorname{grg} g_{t}\left(a_{i}\right)}{T_{k+1}^{\alpha_{j}}}\right) T_{k+1}^{2}-Q_{t_{i}}^{\alpha_{j}}\left(\frac{g r g_{t}\left(a_{i}\right)}{T_{k}^{\alpha_{j}}}\right) T_{k}^{2}\right] .
\end{aligned}
$$

A commonly accepted way to characterize the outage of a network of randomly located interferers is the spatially averaged outage probability, which is found by removing the conditioning on the network geometry. The spatially averaged outage probability could be found numerically via simulation. The simulation would involve randomly generating different network realizations, and computing the conditional outage probability of each, then averaging over many such network realizations. As an example, the solid red line in Fig. 2.3 shows the numerical average of the outage probability over 100 network realization. A more sophisticated numerical technique is given in 35] which does not use simulation and will work for any arbitrary network topology. However, for more regular shaped networks (e.g., circular, annular, or confined within a regular polygon), it is possible to get an analytical, rather than numerical, solution, as we describe in this section.

Let $\mathbb{E}_{\mathbf{X}}\left[F_{S}(s)\right]$ denote the spatially averaged outage probability, where the expectation is with respect to $\mathbf{X}=\left(X_{0}, X_{1}, \ldots, X_{K}\right)$. From 2.11) and the independence of $\left\{Y_{i}\right\}$, the spatially averaged outage probability can be found as follows:

$$
\mathbb{E}_{\mathbf{X}}\left[F_{S}(s)\right]=1-e^{-\eta_{0} s c} \sum_{l=0}^{m_{0}-1} \frac{1}{l !}\left(\eta_{0} s c\right)^{l} \sum_{n=0}^{l}\left(\begin{array}{l}
l \\
t
\end{array}\right) \frac{t !}{c^{t}} \sum_{t_{i} \in \mathcal{T}_{t}} \prod_{i=1}^{K} E_{X_{i}}\left[\gamma_{i}\right]
$$

where

$$
\gamma_{i}=p_{i, 0} \delta\left(t_{i}\right)+\sum_{j=1}^{J} \frac{p_{i, j}}{t_{i} !} \frac{\eta_{i, j}^{m_{i, j}}}{\Gamma\left(m_{i, j}\right)}\left(\eta_{0} s+\eta_{i, j}\right)^{-t_{i}-m_{i, j}} \Gamma\left(t_{i}+m_{i, j}\right) .
$$

If the $X_{i}$ are independent and uniformly distributed on an annulus, then the PDF of $R_{i}=\left|X_{i}\right|$ is $f_{R_{i}}(r)=\frac{2 \pi r}{|A|}$ for $r_{\text {in }} \leq r \leq r_{\text {out }}$, and $\phi_{i}=\angle X_{i}$ is uniform over $(0,2 \pi)$. Since $R_{i}$ is independent of $\phi_{i}$,

$$
\mathbb{E}_{X_{i}}\left[\gamma_{i}\right]=\mathbb{E}_{R_{i}, \phi_{i}}\left[\gamma_{i}\right]=\mathbb{E}_{R_{i}} \mathbb{E}_{\phi_{i}}\left[\gamma_{i}\right]
$$


A key challenge in finding the spatial average is that not only does the power of each interferer depend on the distance $R_{i}$ to the interferer, but the probabilities $p_{i, j}$ can also depend on the distance. This makes the integral required for spatial averaging difficult, if not impossible, to evaluate in closed form. To alleviate this issue, we divide the network $|A|$ into $L$ concentric rings and assume that for sufficiently small rings the probabilities $p_{i, j}$ are constant for all interferers in a given ring. Let $T_{0}=r_{\text {in }}, T_{L}=r_{\text {out }}$ and $T_{k}=r_{\text {in }}+k \Delta r$ for $k=0,1, \ldots, L$ where $\Delta r=\frac{r_{\text {out }}-r_{\text {in }}}{L}$. For large $L$, we use the approximation

$$
p_{b}\left(R_{i}\right) \simeq p_{b}\left(\frac{T_{k+1}+T_{k}}{2}\right) \triangleq p_{b}^{(k)}
$$

for $T_{k} \leq R_{i} \leq T_{k+1}$ and $k=0,1, \ldots, L-1$. Thus, the probability $p_{i, j}$ will be approximated by $p_{i, j}^{(k)}$, for $T_{k} \leq R_{i} \leq T_{k+1}$.

Denoting $g_{r}\left(\phi_{i}\right) g_{t}\left(a_{i}\right)=\gamma_{i, j}$, conditioned on $T_{k} \leq R_{i} \leq T_{k+1}$ and $\phi_{i}$, the conditional PDF of $\Omega_{i, j}$ is

$$
f_{\Omega_{i, j}}\left(\omega \mid T_{k} \leq R_{i} \leq T_{k+1}, \phi_{i}\right)=\frac{2 \omega^{-\frac{2+\alpha_{j}}{\alpha_{j}}}}{\alpha_{j}\left(T_{k+1}^{2}-T_{k}^{2}\right)} \gamma_{i, j}^{\frac{2}{\alpha_{j}}}
$$

for $\gamma_{i, j} / T_{k+1}^{\alpha_{j}} \leq \omega \leq \gamma_{i, j} / T_{k}^{\alpha_{j}}$. Since

$$
P\left[T_{k} \leq R_{i} \leq T_{k+1}\right]=\frac{\pi\left(T_{k+1}^{2}-T_{k}^{2}\right)}{|A|}
$$

the PDF of $\Omega_{i, j}$ conditioned on $\phi_{i}$ is

$$
f_{\Omega_{i, j}}\left(\omega \mid \phi_{i}\right)=\sum_{k=0}^{L-1} \frac{2 \pi}{\alpha_{j}|A|} \omega^{-\frac{2+\alpha_{j}}{\alpha_{j}}} \gamma_{i, j}^{\frac{2}{\alpha_{j}}}\left[u\left(\omega-\frac{\gamma_{i, j}}{T_{k+1}^{\alpha_{j}}}\right)-u\left(\omega-\frac{\gamma_{i, j}}{T_{k}^{\alpha_{j}}}\right)\right] .
$$

over $\gamma_{i, j} / r_{\text {out }}^{\alpha_{j}} \leq \omega \leq \gamma_{i, j} / r_{\text {in }}^{\alpha_{j}}$ and zero elsewhere. The expectation in 2.19 can be evaluated with respect to $\Omega_{i, j}$, i.e,

$$
E_{X_{i}}\left[\gamma_{i}\right]=E_{\phi_{i}} E_{R_{i}}\left[\gamma_{i}\right]=E_{\phi_{i}} E_{\Omega_{i, j}}\left[\gamma_{i}\right]
$$

Substituting (2.18) into 2.25) and using the definition of $\eta_{i, j}$,

$$
E_{X_{i}}\left[\gamma_{i}\right]=E_{\phi_{i}} E_{\Omega_{i}}\left[p_{i, 0} \delta\left(t_{i}\right)+\sum_{j=1}^{J} \frac{p_{i, j}}{t_{i} !} \frac{\Gamma\left(t_{i}+m_{i, j}\right)}{\Gamma\left(m_{i, j}\right)} \frac{\Omega_{i, j}^{t_{i}}}{m_{i, j}^{t_{i}}}\left(1+\frac{\eta_{0} s \Omega_{i, j}}{m_{i, j}}\right)^{-t_{i}-m_{i, j}}\right]
$$


Using the PDF of conditional $\Omega_{i, j}$ in 2.23

$$
\begin{aligned}
& E_{X_{i}}\left[\gamma_{i}\right]=E_{\phi_{i}}\left[p_{i, 0} \delta\left(t_{i}\right)+\sum_{j=1}^{J} \sum_{k=0}^{L-1} \frac{2 \pi \gamma_{i, j}^{\frac{2}{\alpha_{j}}}}{\alpha_{j}|A|} \frac{\Gamma\left(t_{i}+m_{i, j}\right)}{\Gamma\left(m_{i, j}\right) t_{i} !}\right. \\
& \left.\times \frac{p_{i, j}^{(k)}}{m_{i, j}^{t_{i}}} \int_{\frac{\gamma_{i, j}}{T_{k+1}^{\alpha_{j}}}}^{\frac{\gamma_{i, j}}{T_{k}}} \omega^{-\frac{2+\alpha_{j}}{\alpha_{j}}}\left(1+\frac{\eta_{0} s \omega}{m_{i, j}}\right)^{-t_{i}-m_{i, j}} \omega^{t_{i}} d \omega\right]
\end{aligned}
$$

which evaluates to,

$$
\begin{aligned}
& p_{i, 0} \delta\left(t_{i}\right)+\sum_{j=1}^{J} \sum_{k=0}^{L-1} \frac{2 \pi p_{i, j}^{(k)}}{\alpha_{j}|A|} \frac{\Gamma\left(t_{i}+m_{i, j}\right)}{\Gamma\left(m_{i, j}\right) t_{i} !} m_{i, j}^{m_{i, j}}\left(\eta_{0} s\right)^{-m_{i, j}-t_{i}} \\
& \times E_{\phi_{i}}\left[Q_{t_{i}}^{\alpha_{j}}\left(\frac{g_{r}\left(\phi_{i}\right) g_{t}\left(a_{i}\right)}{T_{k+1}^{\alpha_{j}}}\right) T_{k+1}^{2}-Q_{t_{i}}^{\alpha_{j}}\left(\frac{g_{r}\left(\phi_{i}\right) g_{t}\left(a_{i}\right)}{T_{k}^{\alpha_{j}}}\right) T_{k}^{2}\right],
\end{aligned}
$$

where

$$
Q_{t_{i}}^{\alpha_{j}}(x)=\frac{{ }_{2} F_{1}\left(m_{i, j}+t_{i}, m_{i, j}+\frac{2}{\alpha_{j}} ; m_{i, j}+\frac{2}{\alpha_{j}}+1 ;-\frac{m_{i, j}}{x s \eta_{0}}\right)}{x^{m_{i, j}}\left(m_{i, j}+\frac{2}{\alpha_{j}}\right)}
$$

and ${ }_{2} F_{1}(\cdot)$ is the Gauss hypergeometric function. From (2.14), the expected value with respect to $\phi_{i}$ in (2.27), yields equation (2.28) at the top of the page.

Example \#2: This example uses the same parameters as Example \#1 except now the $K=20$ interferers are assumed to be placed randomly within the annulus. Rather than computing the conditional outage probability for just one network realization, Fig. 2.6 shows the spatially averaged outage probability found using (2.17) with $L=10$. In addition to the exact analysis, the spatially averaged outage probability is found using the LOS-ball approximation with two values of $R_{\text {LOS }}: R_{\text {LOS }}=4.4$ corresponding to criterion 1 of 27 and $R_{\mathrm{LOS}}=3.6$, which is the value that, on average, provides the best fit to the exact outage probability. Moreover, the dots on the figure show the spatially averaged outage probability found by averaging analytical expression for conditional outage probability 2.11) over 100 network realizations. Note that the exact analysis provides close agreement with the simulation results, while both values of $R_{\text {LOS }}$ result in a discrepancy. 


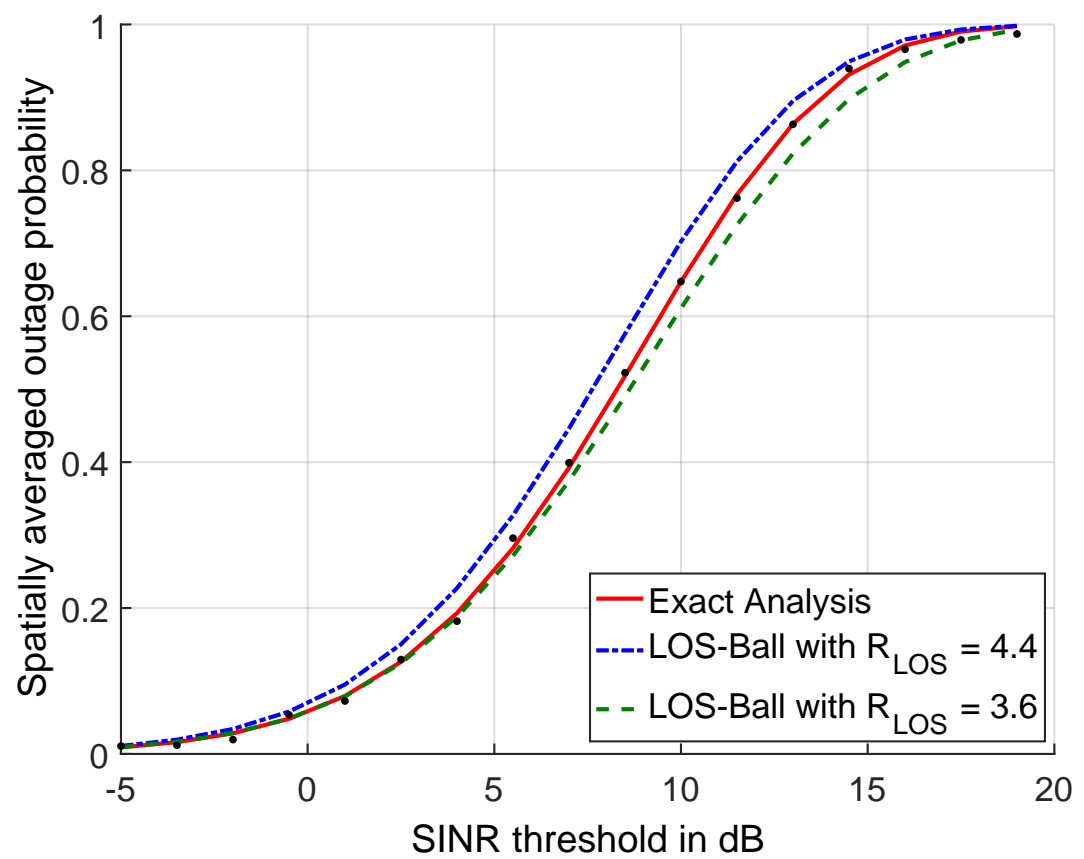

Figure 2.6: Spatially averaged outage probability with $K=20$ randomly located interferers and SNR $=20 \mathrm{~dB}$. In addition to the exact values, the outage found using the LOS-ball approximation and two values of $R_{\text {LOS }}$ are shown. The black dots represents simulation results.

\subsection{Conclusion}

In this chapter, we found analytical expressions that exactly characterize the outage probability in wireless networks when the power of each interferer is selected at random. The set of distributions can correspond to different blockage and directivity states, making it immediately applicable to mmWave systems. Expressions were given for a deterministic (fixed) and random geometries. The work could readily be extended to process other than BPPs, such as Poisson point processes, as well as networks of shapes other than an annulus. Due to space constraints, only a few examples have been shown to confirm the accuracy of the approach; a more detailed analysis could use these expressions to provide insight into the role of various parameters such as the number of interferers $(K)$, array parameters, channel coefficients, and SNR. While the focus on this chapter has been on mmWave, other applications are possible related to cellular networks, distributed MIMO systems, and more elaborate MAC protocols. 


\section{Chapter 3}

\section{The Impact of Correlated Blocking on Millimeter-Wave Personal Networks}

\subsection{Introduction}

Communicating at millimeter-wave (mmWave) frequencies is attractive due to the potential to support high data rates at low latency [2, 3]. The mmWave band is characterized by high attenuation, which is both a blessing and a curse [7]. On the one hand, the desired signal is highly attenuated, and to overcome the attenuation, high gain directional antennas are required; however, due to the small wavelength, compact multi-element antenna arrays are feasible, even on a compact user terminal [36]. On the other hand, interference tends to also be highly attenuated, and thus the band is characterized as having reasonable interference isolation.

Due to these characteristics, mmWave has emerged as a promising solution for wireless personal-area networks (WPAN) and as an enabler for emerging applications such as highresolution untethered virtual reality, augmented reality, and mixed reality 911 .

Another characteristic of mmWave is that it is prone to blockage by objects in the environment, including human bodies [37, 38. Blocking makes it especially difficult to provide universal coverage with a cellular infrastructure. For instance, blockage by walls provides isolation between indoor and outdoor environments, making it difficult for an outdoor base station to provide coverage indoors [5]. 
The performance of mmWave systems can be characterized by the outage probability, or equivalently, by the cumulative distribution function $(\mathrm{CDF})$ of the signal-to-interference ratio (SINR). Alternatively, the performance can be characterized by the coverage probability, which is the complimentary CDF of the SINR, or the rate distribution, which can be found by using information theory to link the SINR to the achievable rate. Prior work has considered the SINR distribution of mmWave personal networks 12 14. Such work assumes that the blockages are drawn from a point process (or, more specifically, that the centers of the blockages are drawn from a point process and each blockage is characterized by either a constant or random width). Meanwhile, the interferers are either in fixed locations or their locations are also drawn from a point process. A universal assumption in this prior art is that the blocking is independent; i.e., each interferer is blocked independently from the other interferers.

In actual networks, blocking might be correlated. This is particularly true when two interferers are close to each other, or more generally when two interferers have a similar angle to the reference receiver. In this case, when one interferer is blocked, there is a significant probability that the other interferer is also blocked. However, correlated blocking can arise even when interferers are not close. Take, for instance, an extreme case where there is just one blockage in the environment and two interferers located far apart from each other. If the first interferer is blocked, then the second one cannot be blocked, giving rise to a negative correlation.

As blocking has a major influence on the distribution of the interference, it must be carefully taken into account. Independent blocking is a crude approximation that fails to accurately capture the true environment, especially when the interferers are closely spaced or when there are few sources of blocking. We note that blocking can be correlated even when the sources of blockage are placed independently according to a point process. The issue of blockage correlation was recently considered in [15, 16], but it was in the context of a localization application where the goal was to ensure that a minimum number of positioning transmitters were visible by the receiver. As such, it was only concerned with the number of unblocked transmissions rather than the distribution of the received aggregate signal (i.e., the interference power). 
In this chapter, we accurately characterize the performance of mmWave WPAN systems in the presence of correlated blocking. We assume that an arbitrary number of blockages are placed according to a point process. For ease of exposition, we consider the case of two dominant interferers, though the methodology can be extended to multiple interferers. The signal model is such that blocked signals are completely attenuated, while line-of-site (LOS), i.e., non-blocked, signals are subject to an exponential path loss and additive white Gaussian noise (AWGN). Though it complicates the exposition and notation, the methodology can be extended to more elaborate models, such as one wherein all signals are subject to fading and NLOS signals are only partially attenuated (see, e.g., [14]).

The remainder of the chapter is organized as follows. We begin by providing the system model in Section 3.2, wherein there are two interferers and an arbitrary number of blockages, each drawn from a binomial point process. Section 3.3 provides an analysis of the SINR distribution, where the results depend on the blockage correlation coefficient. Section 3.4 derives the blockage correlation coefficient using arguments based on the geometry and the properties of the blockage point process; i.e., by using stochastic geometry. Section 3.5 considers furthermore the effects of antenna directivity and randomly placed interferers by allowing the interfering transmitters to have a random location and orientation. The section leverages the analysis provided in 35$]$. Finally, Section 3.6 concludes the chapter, suggesting extensions and generalizations of the work.

\subsection{System Model}

Consider a mmWave WPAN consisting of a reference transmitter-receiver pair surrounded by both blockages and interfering transmitters. The network is contained in an arbitrarily shaped region $A$, where the variable $A$ is used to denote both the region and its area. Any additional sources of interference located outside of $A$ are assumed to be completely attenuated and therefore do not directly factor into the performance of the network, though they may contribute to the noise floor.

The goal of our work is to investigate the influence of correlated blocking on the system performance. While it is possible for there to be more than two sources of interference, and 


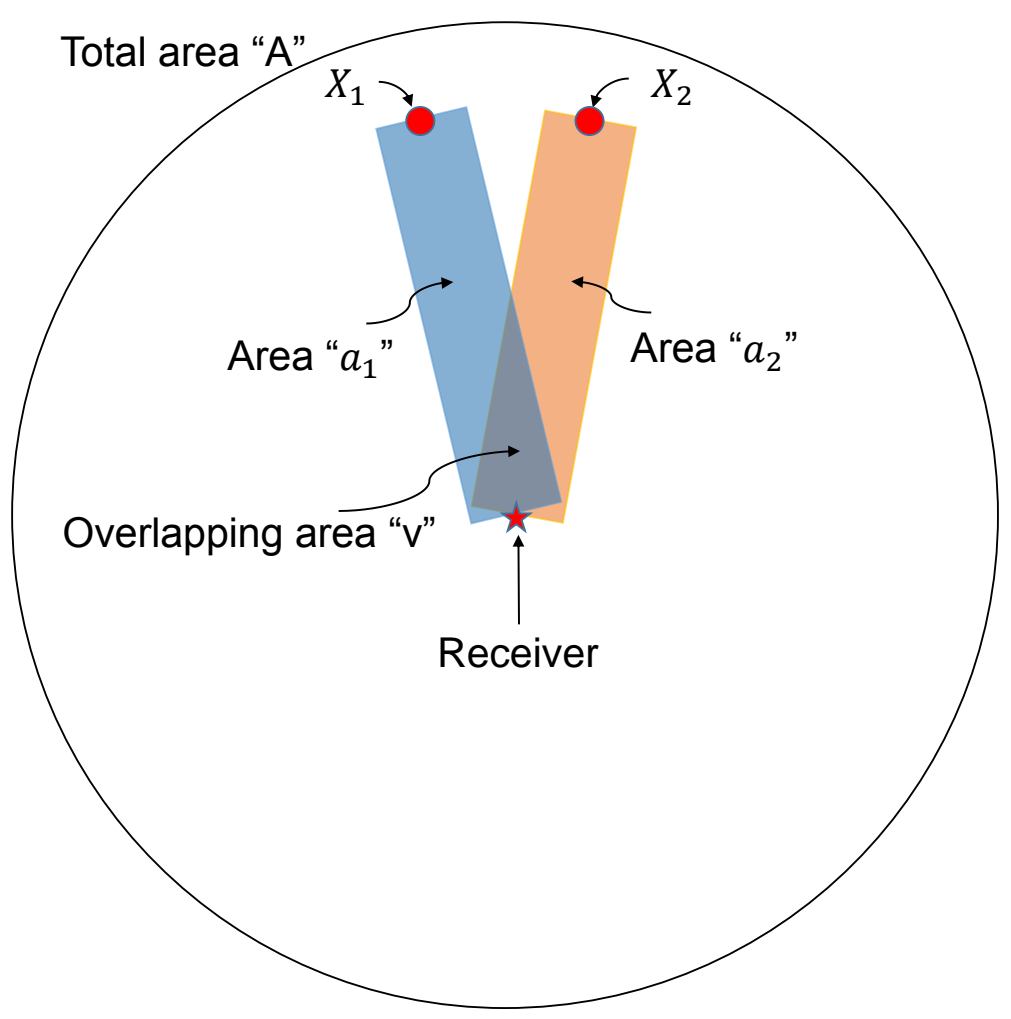

Figure 3.1: Example network topology. Denoted by a red star, the receiver is located at the center of the circular region $A$. Denoted by red dots, the two interferers are in the northern part of $A$. The blocking zones of each interferer are indicated by colored rectangles, and their intersection is represented by $v$.

these interferers may be subject to correlated blocking, the main concept is best exposed by limiting the discussion to just two interferers. As mmWave systems tend to be limited by a few dominant interferers, this limitation is a practical one in most cases. However, Section VI contemplates ways to extend the analysis to the case of multiple interferers.

Hence, there are three transmitters: A source transmitter and two interferers. Let the variable $X_{i}$ denote the transmitter and its location. In particular, let $X_{0}$ denote the source transmitter, and $X_{1}$ and $X_{2}$ denote the interferers. Each location is represented by a complex number $X_{i}=R_{i} e^{j \phi_{i}}$, where $R_{i}$ represents the distance from the receiver to the $i^{\text {th }}$ transmitter and $\phi_{i}$ represents the (azimuth) angle from the receiver to the transmitter $X_{i}$. Without loss of generality, $R_{1} \leqslant R_{2}$. Fig. 3.1 shows an example of the network topology. Here, the network region $A$ is a circle, though our methodology does not require $A$ to be any particular shape. 
The receiver is at the center of $A$ (indicted by the red star), and the two interferers are located in the northern part of $A$ (indicated by the red dots).

Within the network, there are $K$ distributed blockages. As in [16], each blockage is modeled as a point, a line segment of length $W$ centered at the point, and the line segment's orientation angle. The points are distributed according to a binomial point process, and as such, they are independently and uniformly placed on $A$. As in [16], the orientation angles are selected such that the line segment is perpendicular to the line between the receiver and the center of the blockage, which is equivalent to saying that the line segment is actually the projection of the visible face of the blockage rather than the entire object. Although $W$ can itself be random, we assume here that all blockages have the same value of $W$. If a line segment cuts the path between $X_{i}$ and the receiver, then the signal from $X_{i}$ is non line-of-sight (NLOS), while otherwise it is line of sight (LOS). Here, we assume that NLOS signals are completely blocked while LOS signals experience exponential path-loss with a path-loss exponent denoted by $\alpha$.

Each interferer has a blockage region associated with it, indicated by the colored rectangles in Fig. 3.1. We use $a_{i}$ to denote the blockage region associated with $X_{i}, i=\{1,2\}$, and its area. If the center of a blockage falls within $a_{i}$, then $X_{i}$ will be blocked since at least some part of the blockage will intersect the path between $X_{i}$ and the receiver. From the given geometry, it is clear that $a_{i}=W R_{i}$. Unless $X_{1}$ and $X_{2}$ are exactly on opposite sides of $A$, i.e. $\left|\phi_{1}-\phi_{2}\right|=\pi$, there will be an overlapping region $v$ common to both $a_{1}$ and $a_{2}$. Because of the overlap, it is possible for a single blockage to block both $X_{1}$ and $X_{2}$ if the blockage falls within region $v$. This is an example of positive blockage correlation. However, it is also possible to have negative blockage correlation. For instance, if there is just a single blockage (i.e., $K=1$ ), then if the blockage lies in region $a_{1} \backslash v$ (i.e., in $a_{1}$ but not in $v$ ), then it cannot be in $a_{2}$. In this case, $X_{1}$ will be blocked, but $X_{2}$ cannot be blocked. As we will show, negative correlation is also possible even when $K>1$. 


\subsection{SINR Outage Analysis}

The signal-to-interference and noise ratio (SINR) at the receiver is given by

$$
\operatorname{SINR}=\frac{\Omega_{0}}{c+\sum_{i=1}^{2}\left(1-B_{i}\right) \Omega_{i}}
$$

where $\Omega_{i}=R_{i}^{-\alpha}$ is the received power from transmitter $X_{i}$ (recalling that $X_{0}$ is the source transmitter, $X_{1}$ and $X_{2}$ are the two interferers, and $\alpha$ is the path-loss exponent), the constant $c$ is selected so that the signal-to-noise ratio (SNR) is the value of SINR when the interference is turned off (SNR $\left.=\Omega_{0} / c \rightarrow c=\Omega_{0} / \mathrm{SNR}\right)$, and $\left\{B_{1}, B_{2}\right\}$ are a pair of Bernoulli random variables, which may in general be correlated.

The variable $B_{i}$ is used to indicate that $X_{i}$ is blocked, and thus when $B_{i}=1, \Omega_{i}$ does not factor into the SINR. Let $p_{B_{1}, B_{2}}\left(b_{1}, b_{2}\right)$ be the joint probability mass function (pmf) of $\left\{B_{1}, B_{2}\right\}$. Let $p_{i}$ denote the probability that $B_{i}=1$. Furthermore, let $q_{i}=1-p_{i}$. Letting $\rho$ denote the correlation coefficient between $B_{1}$ and $B_{2}$, the joint pmf is found to be

$$
p_{B_{1}, B_{2}}\left(b_{1}, b_{2}\right)= \begin{cases}q_{1} q_{2}+\rho h & \text { for } b_{1}=0, b_{2}=0 \\ q_{1} p_{2}-\rho h & \text { for } b_{1}=0, b_{2}=1 \\ p_{1} q_{2}-\rho h & \text { for } b_{1}=1, b_{2}=0 \\ p_{1} p_{2}+\rho h & \text { for } b_{1}=1, b_{2}=1\end{cases}
$$

where $h=\sqrt{p_{1} p_{2} q_{1} q_{2}}$. A proof of A.12 can be found in 39].

Because blockages are uniformly distributed over $A$, the probability that a given blockage lands in $a_{i}$ is equal to $a_{i} / A$, and hence the probability it is outside $a_{i}$ is $1-a_{i} / A$. Since blockages are independently placed, the probability that all $K$ blockages are outside $a_{i}$ is $\left(1-a_{i} / A\right)^{K}$, and when this occurs, $X_{i}$ will be be LOS (i.e., not blocked). Conversely, $X_{i}$ will be NLOS (i.e., blocked) when not all of the blockages are outside $a_{i}$, which occurs with probability

$$
p_{i}=1-\left(1-\frac{a_{i}}{A}\right)^{K}
$$

The goal of this section to formulate the $\operatorname{CDF}$ of the $\operatorname{SINR} F_{\text {SINR }}(\beta)$, which quantifies the likelihood that the SINR at the receiver is below some threshold $\beta$. If $\beta$ is interpreted as 
the minimum acceptable SINR required to achieve reliable communications, then $F_{\text {SINR }}(\beta)$ is the outage probability of the system $P_{o}(\beta)=F_{\mathrm{SINR}}(\beta)$. The coverage probability is the complimentary $\mathrm{CDF}, P_{c}(\beta)=1-F_{\mathrm{SINR}}(\beta)$ and is the likelihood that the SINR is sufficiently high to provide coverage. The rate distribution can be found by linking the threshold $\beta$ to the transmission rate, for instance by using the appropriate expression for channel capacity.

The CDF of SINR evaluated at threshold $\beta$ can be determined as follows:

$$
\begin{aligned}
F_{\mathrm{SINR}}(\beta) & =P[\operatorname{SINR} \leq \beta] \\
& =1-P[\underbrace{\sum_{i=1}^{2} \Omega_{i}\left(1-B_{i}\right)}_{Z} \leq \frac{\Omega_{0}}{\beta}-c] \\
& =1-F_{Z}\left(\frac{\Omega_{0}}{\beta}-c\right) .
\end{aligned}
$$

The discrete variable $Z$ represents the sum of the unblocked interference. To find the CDF of $Z$ we need to find the probability of each value of $Z$, which is found as follows. The probability that $Z=0$ can be found by noting that $Z=0$ when both $X_{1}$ and $X_{2}$ are blocked. From A.12, this is

$$
p_{Z}(0)=p_{B_{1}, B_{2}}(1,1)=p_{1} p_{2}+\rho h .
$$

The probability that $Z=\Omega_{i}, i \in\{1,2\}$ can be found by noting that $Z=\Omega_{i}$ when only $X_{i}$ is LOS. From A.12, this is

$$
\begin{aligned}
& p_{Z}\left(\Omega_{1}\right)=p_{B_{1}, B_{2}}(0,1)=q_{1} p_{2}-\rho h . \\
& p_{Z}\left(\Omega_{2}\right)=p_{B_{1}, B_{2}}(1,0)=p_{1} q_{2}-\rho h .
\end{aligned}
$$

Finally, by noting that $Z=\Omega_{1}+\Omega_{2}$ when both $X_{1}$ and $X_{2}$ are LOS leads to

$$
p_{Z}\left(\Omega_{1}+\Omega_{2}\right)=p_{B_{1}, B_{2}}(0,0)=q_{1} q_{2}+\rho h
$$




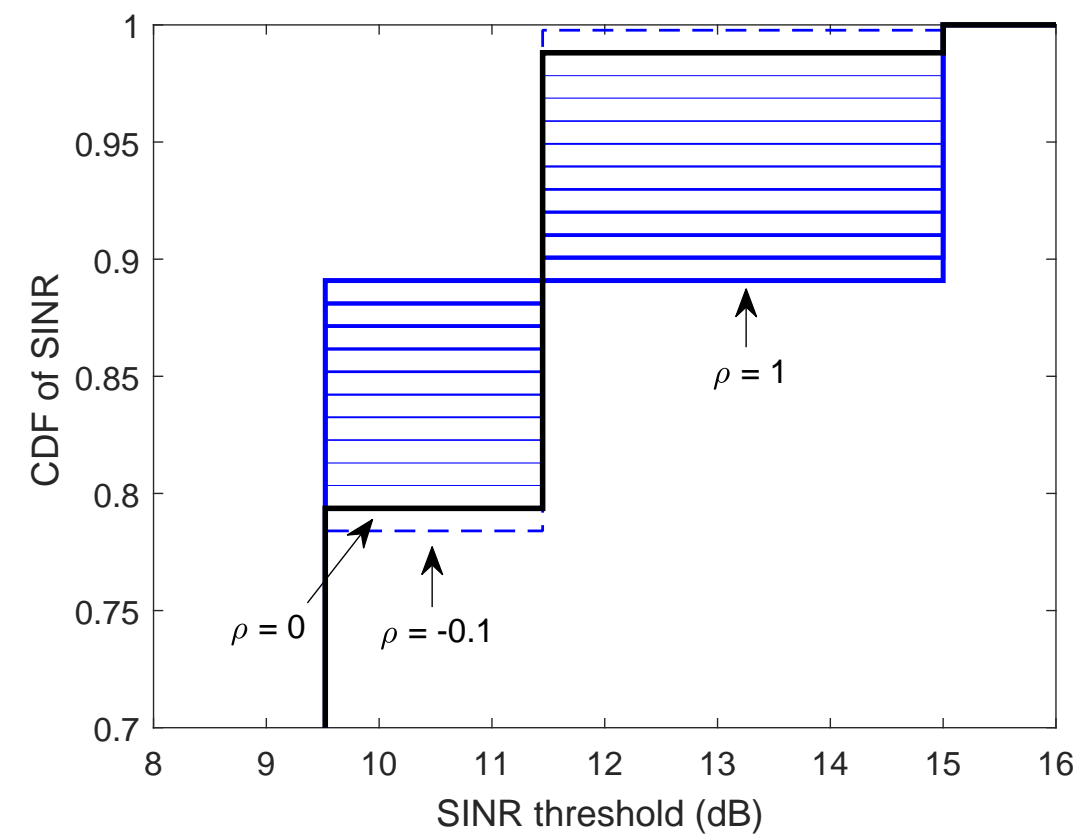

Figure 3.2: The CDF of the $\operatorname{SINR} F_{\text {SINR }}(\beta)$ for different values of $\rho$. The thick black line shows the $\operatorname{CDF}$ when $\rho=0$, the dashed blue line shows the case when $\rho=-0.1$, and the solid blue lines correspond to positive values of $\rho$ in increments of 0.1 .

From (3.5) to 3.8), the CDF of $Z$ is found to be:

$$
F_{Z}(z)= \begin{cases}0 & \text { for } z<0 \\ p_{1} p_{2}+\rho h & \text { for } 0 \leq z<\Omega_{2} \\ p_{1} & \text { for } \Omega_{2} \leq z<\Omega_{1} \\ p_{1}+q_{1} p_{2}-\rho h & \text { for } \Omega_{1} \leq z<\Omega_{1}+\Omega_{2} \\ 1 & \text { for } z \geq \Omega_{1}+\Omega_{2} .\end{cases}
$$

When $R_{1}=R_{2}$, and thus $\Omega_{1}=\Omega_{2}=\Omega, p_{1}=p_{2}=p$, and $q_{1}=q_{2}=q$, the CDF is

$$
F_{Z}(z)= \begin{cases}0 & \text { for } z<0 \\ p^{2}+\rho p q & \text { for } 0 \leq z<\Omega \\ 1-\rho p q-q^{2} & \text { for } \Omega \leq z<2 \Omega \\ 1 & \text { for } z \geq 2 \Omega .\end{cases}
$$

Fig. 3.2 shows the effect that the value of the correlation coefficient $\rho$ has upon the CDF of SINR, which is found by substituting (3.10) into (3.4). The curves were computed 
with $R_{1}=R_{2}=5, \alpha=2$, and SNR $=15 \mathrm{~dB}$. The value of $p$ was computed using (3.3) by assuming $W=1, K=20$, and that $A$ is a circle of radius 6 . The CDF is found assuming values of $\rho$ between $\rho=-0.1$ to $\rho=1$ in increments of 0.1 . The thick black line represents the case that $\rho=0$, corresponding to uncorrelated blocking. The dashed blue line represents the case when $\rho=-0.1$. The solid blue lines correspond to positive values of $\rho$ in increments of 0.1 , where the thinnest line corresponds to $\rho=0.1$ and the thickest line corresponds to $\rho=1$.

Fig. 3.2 shows a first step up at $9.5 \mathrm{~dB}$, and the increment of the step is equal to the probability that both interferers are LOS. The magnitude of the step gets larger as the blocking is more correlated, because (positive) correlation increases the chance that both interferers are $\operatorname{LOS}$ (i.e., $\left.p_{B_{1}, B_{2}}(0,0)\right)$. Negative correlation actually reduces the magnitude of the step. The next step up occurs at $11.5 \mathrm{~dB}$, which is the SINR when just one of the two interferers is blocked. The magnitude of this jump is equal to the probability that just one interferer is blocked, and this magnitude decreases with positive correlation. Finally, there is a step at $15 \mathrm{~dB}$, which corresponds to the case that both interferers are blocked, in which case the SINR equals the SNR. Notice that when $\rho=1$, the two middle steps merge. This is because when $\rho=1$, it is impossible for just one interferer to be blocked, so the curve goes directly from $\mathrm{SINR}=9.5 \mathrm{~dB}$ to $\mathrm{SINR}=15 \mathrm{~dB}$.

\subsection{Blockage Correlation Coefficient}

Let's now consider how to find $\rho$, the blockage correlation coefficient. From A.12,

$$
\rho=\frac{p_{B_{1}, B_{2}}(0,0)-q_{1} q_{2}}{h}
$$

where $p_{B_{1}, B_{2}}(0,0)$ is the probability that both $X_{1}$ and $X_{2}$ are not blocked. Looking at Fig. 3.1. this can occur when all blockages are outside areas $a_{1}$ and $a_{2}$. Taking into account the overlap $v$, this probability is

$$
p_{B_{1}, B_{2}}(0,0)=\left(1-\frac{a_{1}+a_{2}-v}{A}\right)^{K}
$$

Fig. 3.3 and Fig. 3.4 show $\rho$ as a function of the angular separation $(\theta)$ between $X_{1}$ and $X_{2}$, where $\theta=\left|\phi_{1}-\phi_{2}\right|$. As with Fig. 3.2, $R_{1}=R_{2}=5$, and $A=2 \pi 6^{2}$. In Fig. 3.3, a fixed 


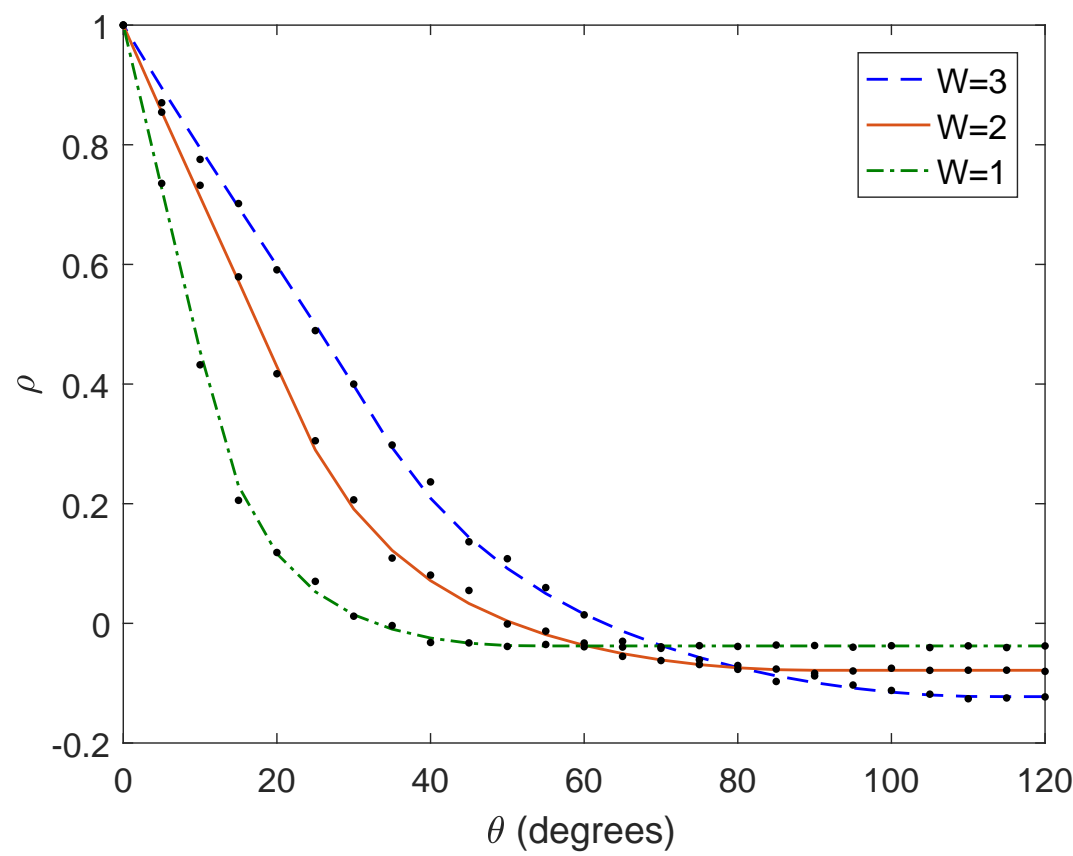

Figure 3.3: The correlation coefficient $(\rho)$ versus the angular separation between $X_{1}$ and $X_{2}\left(\theta=\left|\phi_{1}-\phi_{2}\right|\right)$ for different values of blockage width $(W)$. The black dots represent simulation results. The curves show the exact analytical expression found using the methods of this chapter.

value of $K=1$ is used and $W$ is varied. In Fig. 3.4, $W=3$ and the value of $K$ is varied. In Fig. 3.3, the black dots represents simulation results, which are shown merely to confirm the validity of the approach.

Both figures show that $\rho$ decreases with increasing $\theta$. This is because the area $v$ gets smaller as $\theta$ increases. As $\theta$ approaches $\pi$ radians, $v$ approaches zero, and the correlation is minimized. Note that the minimum value is actually less than zero, showing the possibility of negative correlation. The negative correlation can occur when $a_{1}$ and $a_{2}$ are non-overlapping because if $X_{1}$ is blocked by $m$ blockages, then there are only $K-m$ blockages left that could possibly block $X_{2}$. The figures show that correlation is more dramatic when $W$ is large, since a single large blockage is likely to simultaneously block both interferers, and when $K$ is small, which corresponds to the case that there are fewer blockages.

Fig. 3.5 shows the CDF of the SINR for the same network with different values of $W$ and $K$. The area $A$ is again a circle with radius $6, R_{1}=R_{2}=5, \mathrm{SNR}=15 \mathrm{~dB}$ and $\theta=25^{\circ}$. The solid blue line shows the SINR distribution found by accurately accounting 


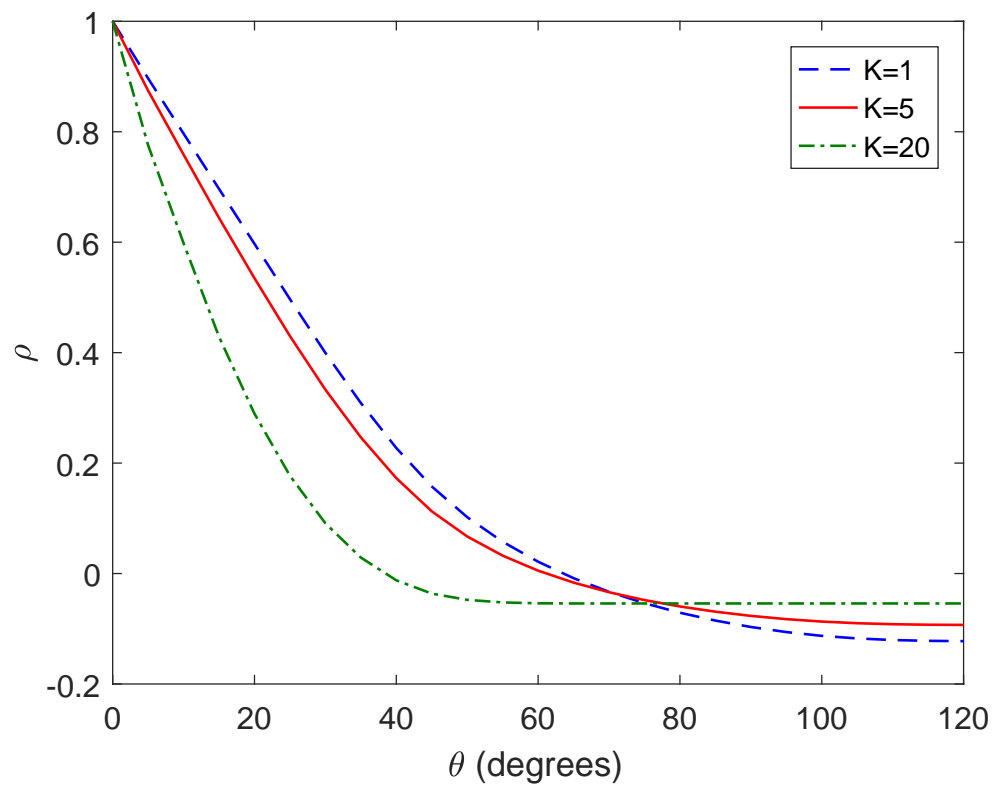

Figure 3.4: The correlation coefficient $(\rho)$ versus the angular separation between $X_{1}$ and $X_{2}$ $\left(\theta=\left|\phi_{1}-\phi_{2}\right|\right)$ for different values of number of blockages $(K)$.

for correlated blocking; i.e., by using the methodology of this section to find $\rho$. The dashed red line corresponds to the $\mathrm{CDF}$ assuming blocking is independent; i.e., fixing $\rho=0$. From (3.10), the difference between the two curves is equal to $\rho p q$, and thus the difference will grow if $\rho$ increases and/or $p$ increases, as long as $p<0.5$. By comparing Fig. 3.5(a) to Fig. 3.5(b) or Fig. 3.5(c) to Fig. 3.5(d), the gap between the two CDFs increases as $W$ goes from 2 to 3. This can be explained by referring to Fig. 3.3, which shows that $\rho$ increases for sufficiently small $\theta$ as $W$ increases. By comparing Fig. 3.5(a) to Fig. 3.5(c) or Fig. 3.5(b) to Fig. 3.5(d), the gap between the two CDFs increases as $K$ goes from 2 to 5 . Although Fig. 3.4 shows that $\rho$ decreases with increasing $K$ at moderate $\theta$, this behavior is offset by the fact that $p$ increases with $K$, per (3.3). Thus the gap actually increases with increasing $K$.

\subsection{Antenna Directivity and Spatial Randomness}

Thus far, we have assumed that the interferers are in fixed locations and the antennas are omnidirectional. In practice, the locations of the interferers may themselves be random, and directional antennas may be used. When the antennas are directional, the received power 


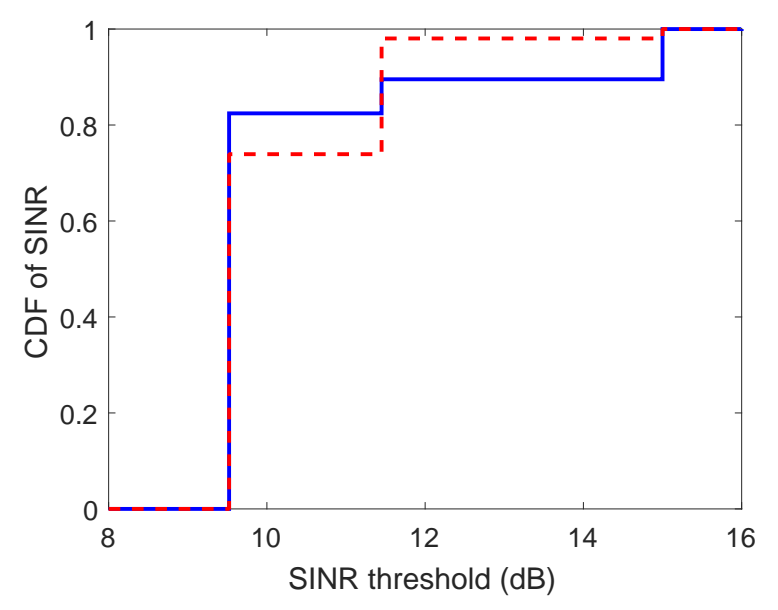

(a) $K=2$ and $W=2$

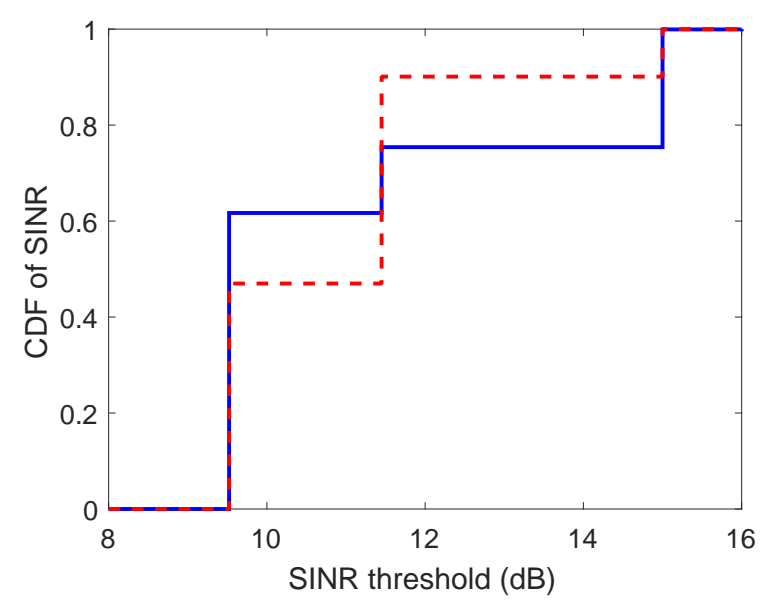

(c) $K=5$ and $W=2$

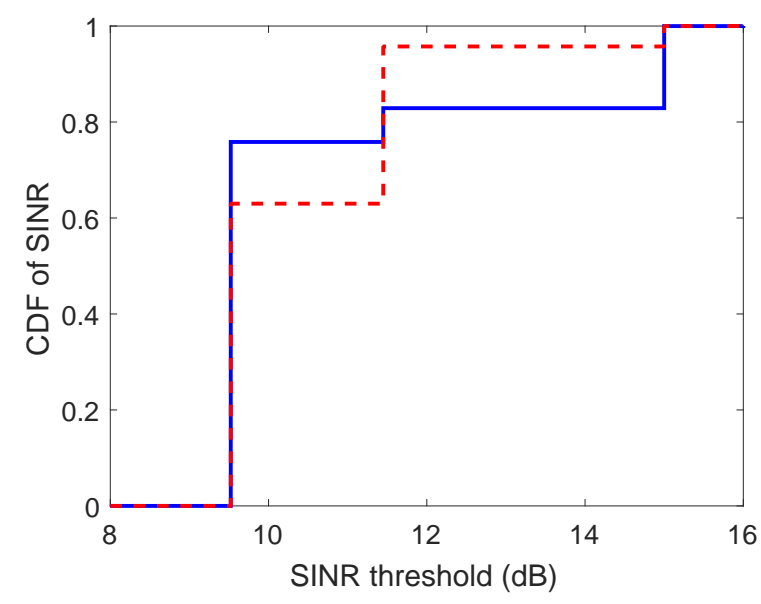

(b) $K=2$ and $W=3$

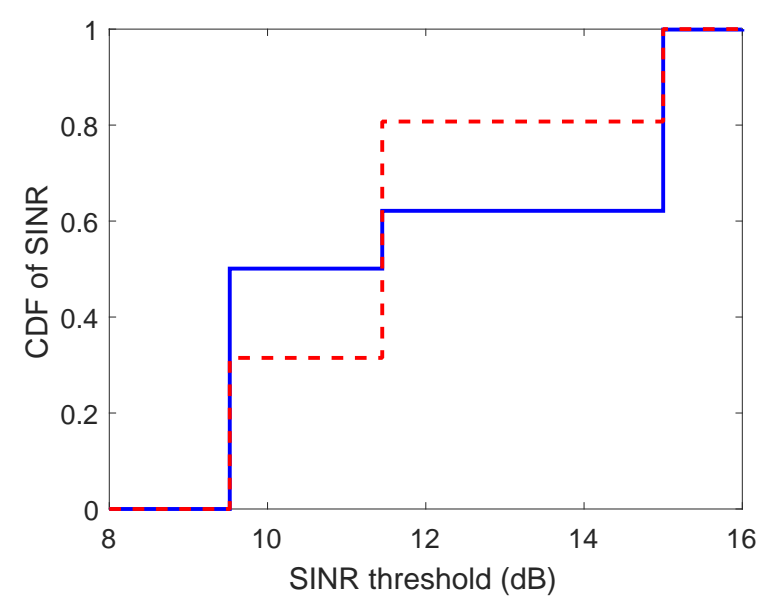

(d) $K=5$ and $W=3$

Figure 3.5: Comparison of CDF of SINR that accounts for correlated blockage (solid blue line) against independent blockage (dotted red line).

from the $i^{\text {th }}$ interferer is

$$
\Omega_{i}=g_{r}\left(\phi_{i}\right) g_{t}\left(\left|\phi_{i}-\psi_{i}\right|-\pi\right) R_{i}^{-\alpha}
$$

where $g_{r}($.$) is the antenna gain of the receiver, g_{t}($.$) is the antenna gain of the transmitter$ and $\psi_{i}$ is the azimuth angle of interferer's transmit antenna. Here, we assume the antenna patterns are function of the azimuth angle only. The red solid line in Fig. 3.6 shows an example antenna pattern for a 4-element planar array [40].

Often, in the mmWave literature, the exact antenna pattern is approximated with a sectorized model, where the antenna gain is just one of two values corresponding to the 
main lobe and the side lobe. The blue dashed line in Fig. 3.6 shows a sectorized antenna that approximates the actual antenna pattern 12, 13.

The direction $\psi_{i}$ that $X_{i}$ is pointing is generally unknown and may be modeled as a random variable. Since $\Omega_{i}$ is a function of $\psi_{i}$, it is then a random variable, even if $X_{i}$ is in a fixed location. For a WPAN, we may assume $\psi_{i}$ is uniformly distributed from 0 to $2 \pi$ radians; i.e, $\psi_{i} \sim U(0,2 \pi)$.

Fig. 3.7 shows performance when antenna directivity is taken into account. Two pairs of curves are shown. The first pair (in red) shows the CDF when the actual antenna pattern of Fig. 3.6 is used. The second pair (in blue) shows the CDF when the sectorized model of Fig. 3.6 is used as an approximation. For each pair of curves, one curve (with a solid line) shows the CDF when the blockage correlation is taken into account and the other curve (with the dashed line) shows the CDF when the blocking is assumed to be independent. The curves are generated by again assuming that $A$ is a circle of radius 6 and an $\mathrm{SNR}=15 \mathrm{~dB}$. There are $K=5$ blockages of width $W=2$. The interferers are in fixed locations with $R_{1}=4$, $R_{2}=5$, and $\theta=25^{\circ}$. We note that there is a significant difference in this case between the CDFs predicted using the actual antenna model vs. the curves generated using the sectorized approximation. To a lesser extent, there is a difference for each antenna model between the curve that accounts for correlation and the one that assumes independent blocking, and the difference is more pronounced at higher SINR thresholds.

If, in addition, the location of the $X_{i}$ are random, then each $\Omega_{i}$ is a random variable that depends on both the location and directivity of the interferers. Fig. 3.8 uses the same parameters that were used in Fig. 3.7 except that now the interferers are randomly placed. In particular, the two interferers are placed independently and uniformly within the circular area $A$. The CDF is found by averaging over 1000 such placements (i.e., network realizations). When the locations are random, the difference between the dashed and solid curves begins to tighten up, implying that the effect of correlation is less important. This is because when randomly placed, the two interferers are often far apart from one another. However, correlation is important for certain regions of the plot, particularly at high values of threshold. Moreover, the difference between the two antenna models is less pronounced, especially at lower values of SINR threshold. 


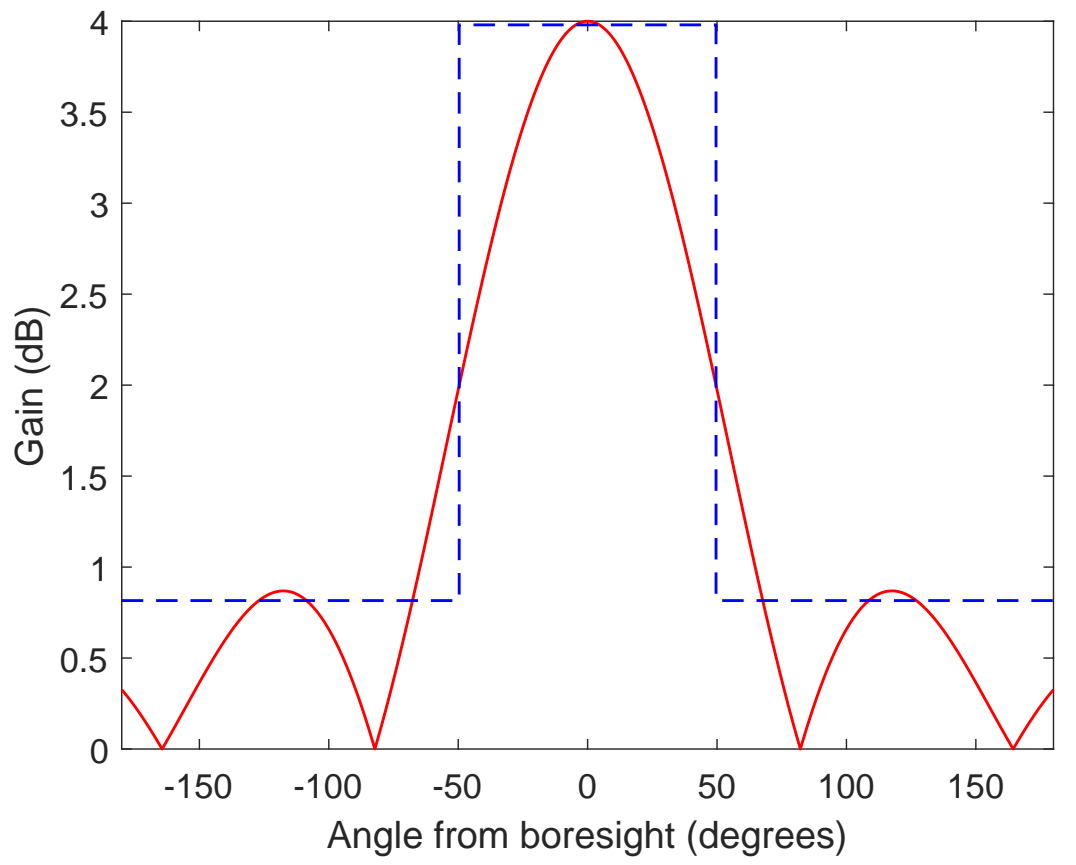

Figure 3.6: Actual antenna model (solid red line) versus sectorized antenna model (dashed blue line).

\subsection{Conclusion}

In a mmWave WPAN system, the interference may be subject to correlated blocking. This is true even if the individual blockages are independently placed, since it is possible for a single blockage to block multiple interferers if the blockage is sufficiently wide and the interferers sufficiently close. The commonly held assumption of independent blocking leads to an incorrect characterization of the performance of the system, for instance, when it is quantified by the distribution of the SINR. By using the methodology in this chapter, the correlation between two sources of interference may be found and factored into the analysis, yielding more accurate results. 


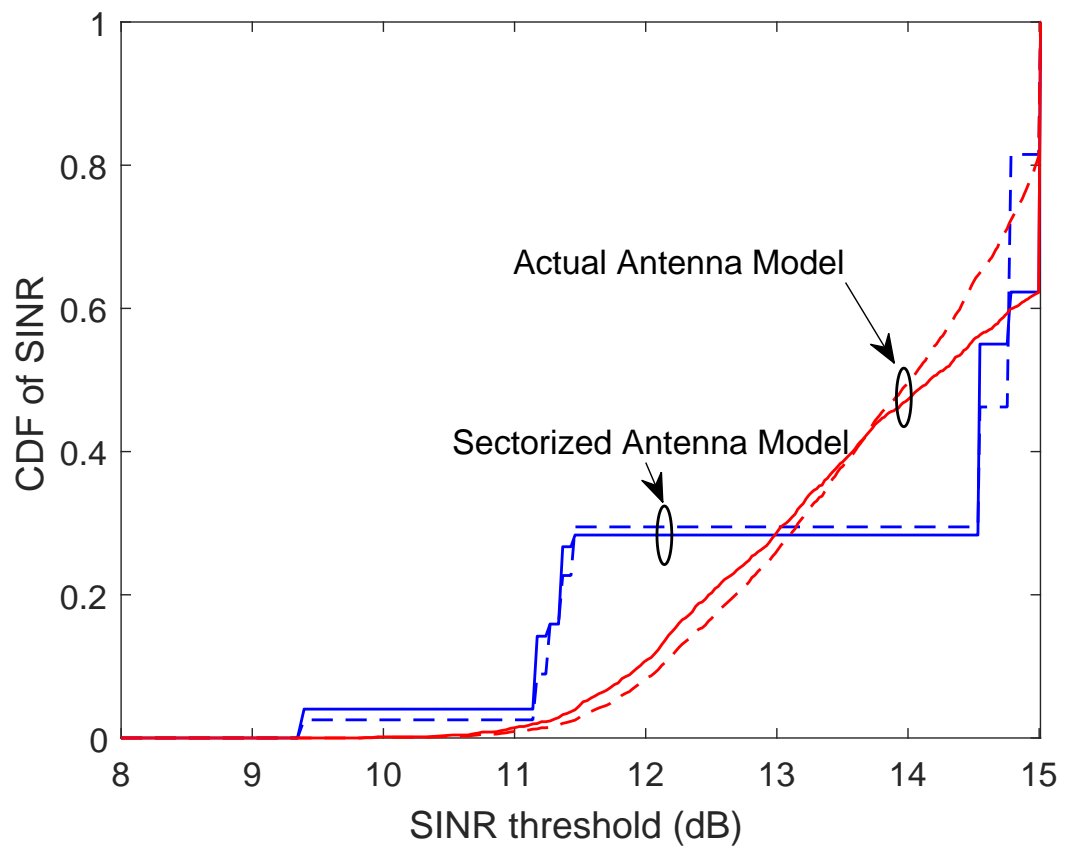

Figure 3.7: Comparison of the CDF of SINR for a particular network realization with the actual antenna pattern (red curves) vs. with a sectorized antenna model (blue curves). Solid lines account for correlated blocking while dashed lines assume independent blocking.

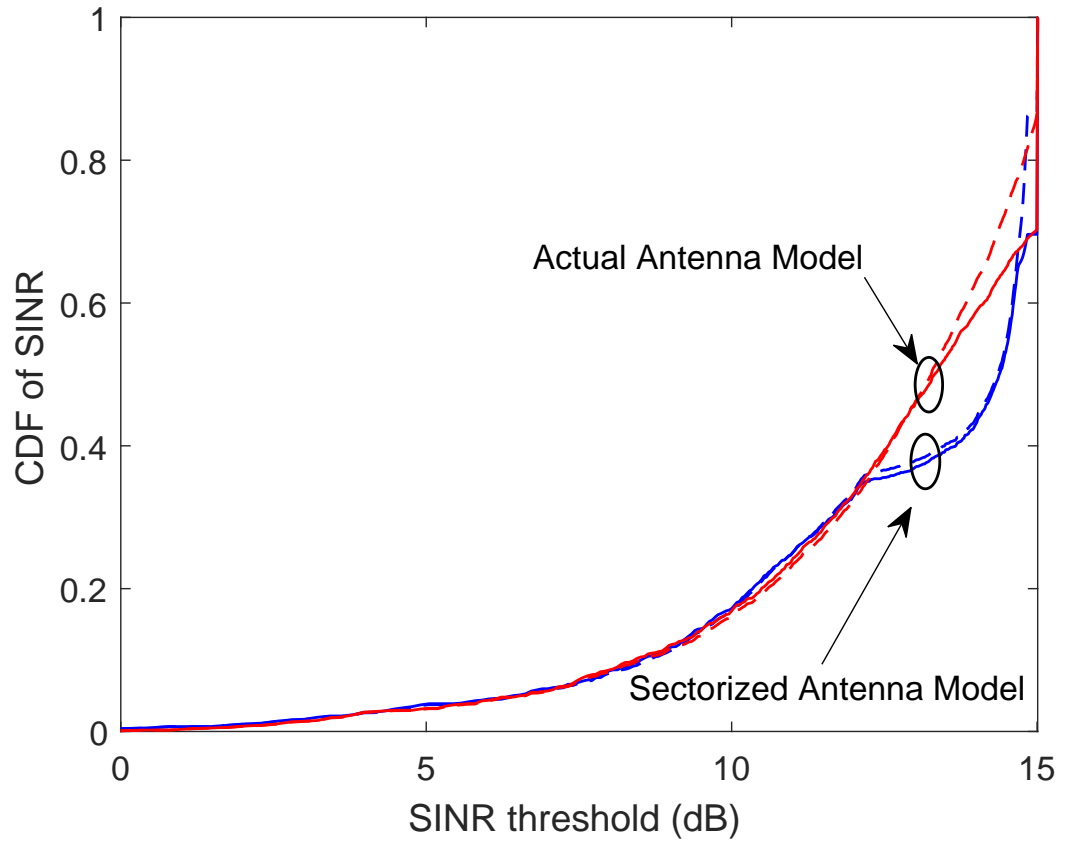

Figure 3.8: The CDF of SINR when averaged over 1000 network network realizations. Red curves use an actual antenna pattern and blue curves use a sectorized antenna model. Solid lines account for correlated blocking while dashed lines assume independent blocking. 


\section{Chapter 4}

\section{Correlated Blocking in}

\section{Millimeter-Wave Cellular Networks: Macrodiversity, Outage, and \\ Interference}

\subsection{Introduction}

At mmWave frequencies, signals are prone to blocking by objects intersecting the paths and severely reducing the signal strength, and thus the Signal to Noise Ratio (SNR) 7 , 9 11. For instance, millimeter-wave (mmWave) has emerged in recent years as a viable candidate for infrastructure-Mbased (i.e., cellular) systems [2,3, 30, 41, 42]. Communicating at mmWave frequencies is attractive due to the potential to support high data rates at low latency [2]. For instance, blocking by walls provides isolation between indoor and outdoor environments, making it difficult for an outdoor base station to provide coverage indoors [5]. To mitigate the issue of blocking in mmWave cellular networks, macrodiversity has emerged as a promising solution, where the user attempts to connect to multiple base stations [43]. With macrodiversity, the probability of having at least one line-of-sight (LOS) path to a base station increases, which can improve the system performance $22,44,45$.

An effective methodology to study wireless systems in general, and mmWave systems in 
particular, is to embrace the tools of stochastic geometry to analyze the SNR and interference in the network $13,14,22,30,46,48$. With stochastic geometry, the locations of base stations and blockages are assumed to be drawn from an appropriate point process, such as a Poisson point process (PPP) [49,50]. When blocking is modeled as a random process, the probability that a link is LOS is an exponentially decaying function of link distance. While many papers assume that blocking is independent [5, 14,51], in reality the blocking of multiple paths may be correlated [46]. The correlation effects are especially important for macrodiverity networks when base stations are close to each other, or more generally when base stations have a similar angle to the transmitter. In this case, when one base station is blocked, there is a significant probability that another base station is also blocked [22, 44, 45].

Prior work has considered the SINR distribution of mmWave personal networks 12 14. Such work assumes that the blockages are drawn from a point process (or, more specifically, that the centers of the blockages are drawn from a point process and each blockage is characterized by either a constant or random width). Meanwhile, the transmitters are either in fixed locations or their locations are also drawn from a point process. A universal assumption in this prior art is that the blocking is independent; i.e., each transmitter is blocked independently from the other transmitters. As blocking has a major influence on the distribution of signals, it must be carefully taken into account. Independent blocking is a crude approximation that fails to accurately capture the true environment, especially when the base stations, or, alternatively, the user equipments (UEs), are closely spaced in the angular domain or when there are few sources of blocking. We note that blocking can be correlated even when the sources of blockage are placed independently according to a point process.

The issue of blockage correlation was considered in [15, 16, 52, 53, but it was in the context of a localization application where the goal was to ensure that a minimum number of positioning transmitters were visible by the receiver. As such, this prior work was only concerned with the number of unblocked transmissions rather than the distribution of the received aggregate signal (i.e., source or interference power). In 46] correlated blocking between interferers was considered for wireless personal area network. Recently correlation between base stations was considered in [44, 45] for infrastructure-based networks with 
macrodiversity, but in these references the only performance metric considered is the $n^{\text {th }}$ order LOS probability; i.e., the probability that at least one of the $\mathrm{n}$ closest base stations is LOS. However, a full characterization of performance requires other important performance metrics, including the distributions of the SNR and, when there is interference, the Signal to Interference and Noise Ratio (SINR) [54]. Alternatively, the performance can be characterized by the coverage probability, which is the complimentary cumulative distribution function of the SNR or SINR, or the rate distribution, which can be found by using information theory to link the SNR or SINR to the achievable rate.

In this chapter, we propose a novel approach for fully characterizing the performance of macrodiversity in the presence of correlated blocking. While, like 44, 45, we are able to characterize the spatially averaged LOS probability (i.e., the LOS probability averaged over many network realizations), our analysis shows the distribution of the LOS probability, which is the fraction of network realizations that can guarantee a threshold LOS probability rather than its mere spatial average. Moreover, we are able to similarly capture the distributions of the SNR and SINR and validate our framework by comparing the analysis to a real data building model. Furthermore we extended the study to more general case of an arbitrary macrodiversity order, and identified the minimum macrodiversity order required to achieve desired performance in the presence of interference.

We assume that the centers of the blockages are placed according to a PPP. We first analyze the distributions of LOS probability for first- and second-order macrodiversity. We then consider the distribution of SNR and SINR for the cellular uplink with both selection combining and diversity combining. The signal model is such that blocked signals are completely attenuated, while LOS, i.e., non-blocked, signals are subject to an exponential path loss and additive white Gaussian noise (AWGN). Though it complicates the exposition and notation, the methodology can be extended to more elaborate models, such as one wherein all signals are subject to fading and non-LOS (NLOS) signals are partially attenuated (see, e.g., [14]).

The remainder of the chapter is organized as follows. We begin by providing the system model in Section 4.2, wherein there are base stations and blockages, each drawn from a PPP. In Section 4.3 we provide an analysis of the LOS probability under correlated blocking 
and derive the blockage correlation coefficient using arguments based on the geometry and the properties of the blockage point process; i.e., by using stochastic geometry. Section 4.4 provides a framework of the distribution of SNR, where the results depend on the blockage correlation coefficient. In Section 4.5, we validate our framework by comparing the analysis to a real data model. Then in Section 4.6, interference is considered and the SINR distribution is formalized. Finally, Section 4.7 concludes the chapter, suggesting extensions and generalizations of the work.

\subsection{System Model}

\subsubsection{Network Topology}

Consider a mmWave cellular network consisting of base stations, blockages, and a source transmitter, which is a UE. The UE attempts to connect to the $\mathrm{N}$ closest base stations, and therefore operates in a $N^{t h}$ order macrodiversity mode. The locations of the base stations are modeled as an infinite homogeneous PPP with density $\lambda_{b s}$. We assume the centers of the blockages also form a homogeneous PPP with density $\lambda_{b l}$, independent from the base station process. Let $Y_{0}$ indicate the source transmitter and its location. Due to the stationarity of the PPPs, and without loss of generality, we can assume the coordinates are chosen such that the source transmitter is located at the origin; i.e., $Y_{0}=0$. In Section 4.6, we will consider additional transmitters located in neighboring cells, which act as interferers.

Let $X_{i}$ for $\mathrm{i} \in \mathbb{Z}^{+}$denote the base stations and their locations. Let $R_{i}=\left|X_{i}\right|$ be the distance from $Y_{0}$ to $X_{i}$. Base stations are ordered according to their distances to $Y_{0}$ such that $R_{1} \leq R_{2} \leq \ldots$. The signal of the source transmitter is received at the closest $N$ base stations, and hence, $N$ is the number of $X_{i}$ connected to $Y_{0}$. For a PPP, a derivation of the distribution of $R_{1}, \ldots, R_{N}$ is given in Appendix $\mathrm{B}$, which implies a methodology for generating these distances within a simulation.

Fig. 4.1 shows an example of second-order macrodiversity $(N=2)$ cellular network where the user attempts to connect to its closest two base stations. The solid line indicates the link from the user to the base station is LOS, while the dashed line indicates the link is NLOS. 


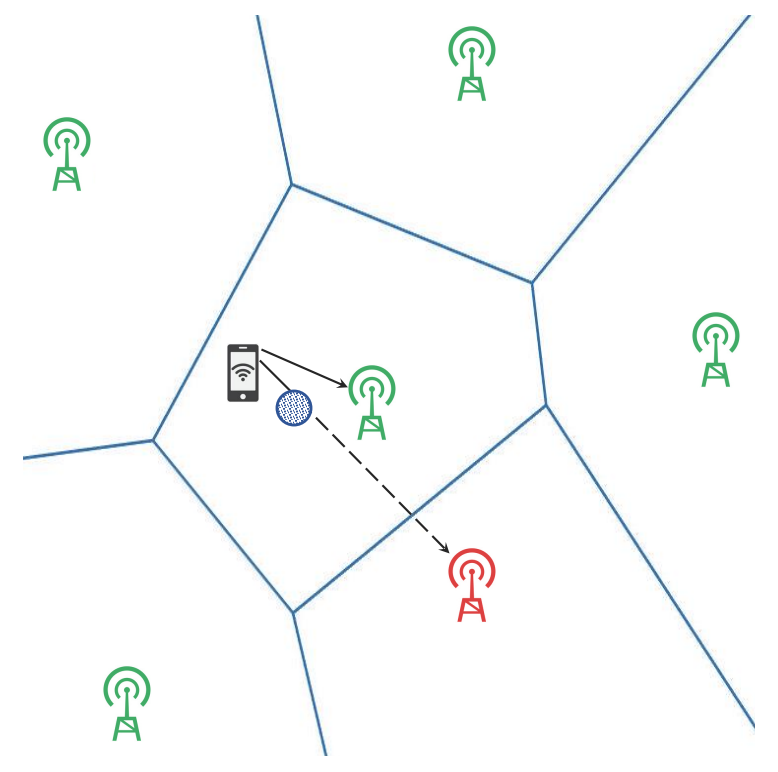

(a) One base station is blocked.

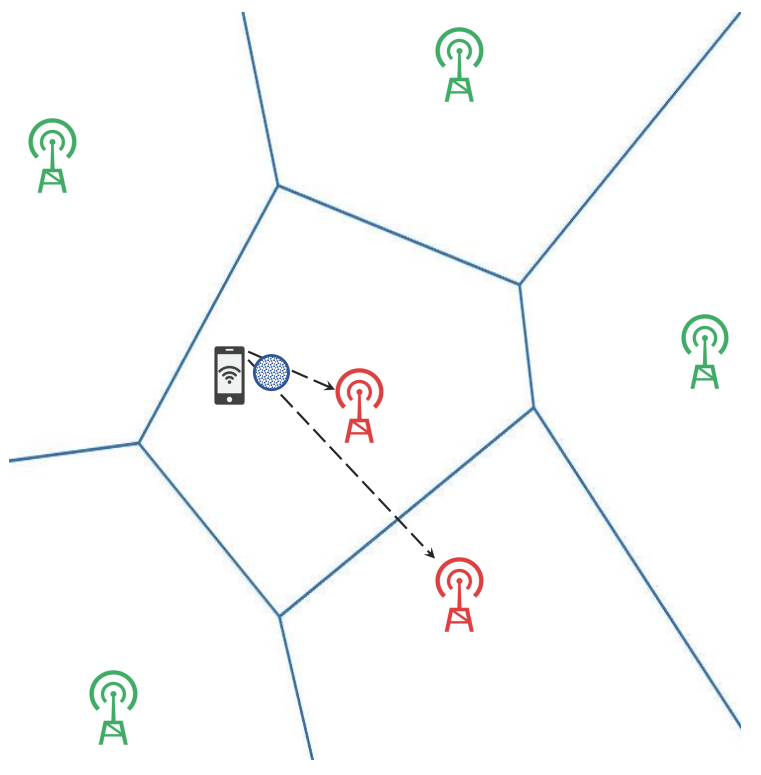

(b) Closest two base stations are blocked.

Figure 4.1: Example network topology with two different blockage scenarios. The source transmitter is the mobile device shown in the central cell. Its signal is transmitted to its closest two base stations. The solid line indicates the link is LOS, while the dashed line indicates the link is NLOS.

The figure shows examples of two different blockage scenarios. In Fig. 4.1(a) the closest base station $\left(X_{1}\right)$ is LOS while $X_{2}$ is NLOS to the user, in which case the blockage only blocks a single link. In Fig. 4.1(b) a single blockage blocks both links to $X_{1}$ and $X_{2}$. The fact that sometimes a single blockage can block both links is an illustration of the effect of correlated blocking.

\subsubsection{Blockage Model}

As in [46], each blockage is a segment of length $W$. To capture the worst-case scenario, as shown in Fig. 4.2(a), it is assumed that the line representing the blockage is perpendicular to the line that connects it to the transmitter. Although $W$ can itself be random as in [44], we assume here that all blockages have the same value of $W$. In Fig. 4.2(a), the red stars indicate the blocked base stations, which are located in the blue shaded region. If a blockage cuts the path from $Y_{0}$ to $X_{i}$, then the signal from $Y_{0}$ is NLOS, while otherwise it is LOS. Here, we assume that NLOS signals are completely blocked while LOS signals experience exponential 


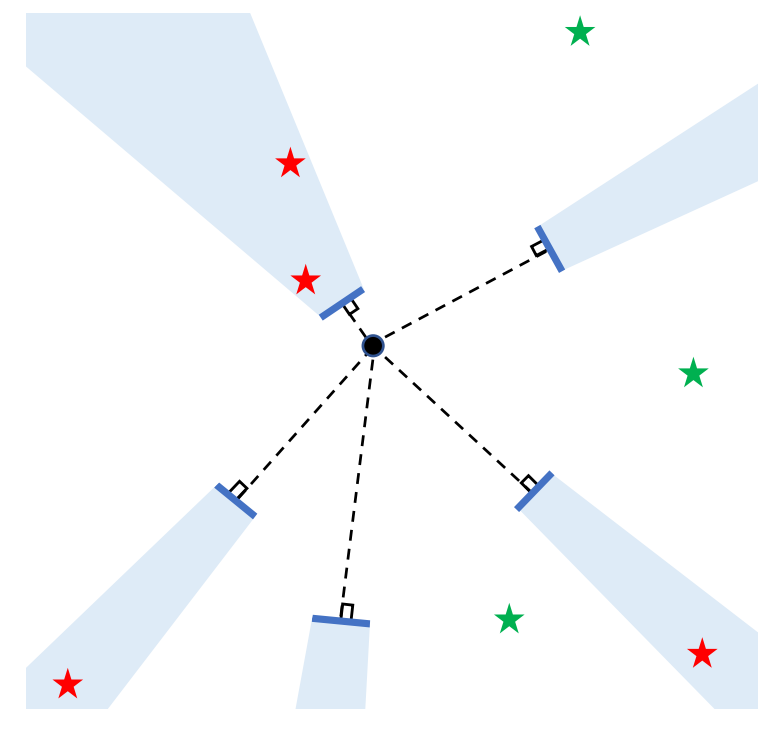

(a)

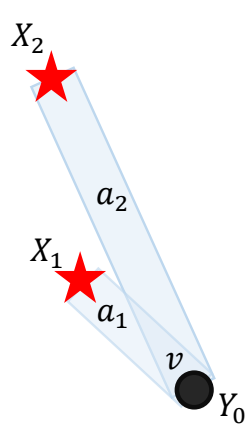

(b)

Figure 4.2: Illustration of the blockage model. (a) Network example consisting of base stations indicated by the stars and blockages indicated by blue lines surrounding the transmitter, which is indicated by the black circle. The blockages are modeled as a line of length $\mathrm{W}$ facing the transmitter; (b) Equivalent blockage regions. $a_{1}$ and $a_{2}$ are the blockage areas, and $\mathrm{v}$ is the overlapping area.

path-loss with a path-loss exponent $\alpha$; i.e., the power received by $X_{i}$ is proportional to $R_{i}^{-\alpha}$.

Each base station has a blockage region associated with it, illustrated by the blue shaded rectangles shown in Fig. 4.2(b). We use $a_{i}$ to denote the blockage region associated with $X_{i}$ and its area; i.e., $a_{i}$ is both a region and an area. If the center of a blockage falls within $a_{i}$, then $X_{i}$ will be blocked since at least some part of the blockage will intersect the path between $X_{i}$ and $Y_{0}$. Because $a_{i}$ is a rectangle of length $R_{i}$ and width $W$, it is clear that $a_{i}=W R_{i}$. Unless $X_{1}$ and $X_{2}$ are exactly on opposite sides of the region, there will be an overlapping region $v$ common to both $a_{1}$ and $a_{2}$. Because of the overlap, it is possible for a single blockage to simultaneously block both $X_{1}$ and $X_{2}$ if the blockage falls within region $v$, which corresponds to correlated blocking. 


\subsection{LOS Probability Analysis under Correlated Block- ing}

In this section, we analyze the LOS probability, which is denoted $p_{L O S}$, and the impact of blockage correlation. Our focus is on second-order macrodiversity, where the signal of the source transmitter $Y_{0}$ is received at the two closest base stations $X_{1}$ and $X_{2}$. The LOS probability is the probability that at least one $X_{i}$ is LOS to the transmitter. Because the base stations are randomly located, the value of $p_{L O S}$ will vary from one network realization to the next, or equivalently by a change of coordinates, from one source transmitter location to the next. Hence $p_{L O S}$ is itself a random variable and must be described by a distribution. To determine $p_{L O S}$ and its distribution, we first need to define the variable $B_{i}$ which indicates that the path between $Y_{0}$ and $X_{i}$ is blocked. Let $p_{B_{1}, B_{2}}\left(b_{1}, b_{2}\right)$ be the joint probability mass function (pmf) of $\left\{B_{1}, B_{2}\right\}$. Let $p_{i}$ denote the probability that $B_{i}=1$, which indicates the link from $Y_{0}$ to $X_{i}$ is NLOS. Furthermore, let $q_{i}=1-p_{i}$, which is the probability that the link is LOS, and $\rho$ denote the correlation coefficient between $B_{1}$ and $B_{2}$. As shown in Appendix A, the joint pmf of $\left\{B_{1}, B_{2}\right\}$ as a function of $\rho$ found to be

$$
p_{B_{1}, B_{2}}\left(b_{1}, b_{2}\right)= \begin{cases}q_{1} q_{2}+\rho h & \text { for } b_{1}=0, b_{2}=0 \\ q_{1} p_{2}-\rho h & \text { for } b_{1}=0, b_{2}=1 \\ p_{1} q_{2}-\rho h & \text { for } b_{1}=1, b_{2}=0 \\ p_{1} p_{2}+\rho h & \text { for } b_{1}=1, b_{2}=1\end{cases}
$$

where $h=\sqrt{p_{1} p_{2} q_{1} q_{2}}$.

For a two-dimensional homogeneous PPP with density $\lambda$, the number of points within an area $a$ is Poisson with mean $\lambda a$ [55]. From the probability mass function of a Poisson variable, the probability of $k$ points within the area is given by 55

$$
p_{K}(k)=\frac{(\lambda a)^{k}}{k !} e^{-\lambda a}
$$

The event that the path to $X_{i}$ is not blocked (LOS) by an object falling in area $a_{i}$ can be obtained by the void probability of PPP, which is the probability that there are no blockages located in $a_{i}$, or equivalently, the probability that $k=0$. Thus, $q_{i}$, which is equal to the void 
probability, is given by substituting $k=0$ into 4.2 with $\lambda=\lambda_{b l}$ and $a=a_{i}$, which results in

$$
q_{i}=\exp \left(-\lambda_{b l} a_{i}\right)
$$

For first-order macrodiversity $(N=1)$, the LOS probability is given by $q_{1}$. Conversely, $X_{i}$ will be NLOS when at least one blockage lands in $a_{i}$ and this occurs with probability $p_{i}=1-q_{i}$ given by

$$
p_{i}=1-\exp \left(-\lambda_{b l} a_{i}\right)
$$

For second-order macrodiversity $(\mathrm{N}=2)$, there will be a LOS signal as long as both paths are not blocked. This corresponds to the case that $B_{1}$ and $B_{2}$ are both not equal to unity. When blocking is not correlated, the corresponding LOS probability is $1-p_{1} p_{2}$. Correlated blocking may be taken into account by using (4.1) and noting that the LOS probability is the probability that $B_{1}$ and $B_{2}$ are not both equal to one, which is given by

$$
p_{L O S}=1-p_{B_{1}, B_{2}}(1,1)=1-p_{1} p_{2}-\rho h
$$

The blockage correlation coefficient $\rho$ can be found from 4.1,

$$
\rho=\frac{p_{B_{1}, B_{2}}(0,0)-q_{1} q_{2}}{h}
$$

where $p_{B_{1}, B_{2}}(0,0)$ is the probability that both $X_{1}$ and $X_{2}$ are LOS. Looking at Fig. 4.2(b), this can occur when there are no blockages inside $a_{1}$ and $a_{2}$. Taking into account the overlap $v$, this probability is the void probability for area $\left(a_{1}+a_{2}-v\right)$, which is given by

$$
p_{B_{1}, B_{2}}(0,0)=e^{-\lambda_{b l}\left(a_{1}+a_{2}-v\right)}
$$

Details on how to compute the overlapping area $v$ are provided in 46. Substituting (4.7) into (4.6) into (4.5) and using the definitions of $p_{i}$ and $q_{i}$, yields

$$
p_{L O S}=e^{-\lambda_{b l} a_{1}}+e^{-\lambda_{b l} a_{2}}-e^{-\lambda_{b l}\left(a_{1}+a_{2}-v\right)}
$$

Let $\theta$ be the angular separation between $X_{1}$ and $X_{2}$. The relationship between the angular separation $\theta$ and the correlation coefficient $\rho$ is illustrated in Fig. 4.3 using an example. In 


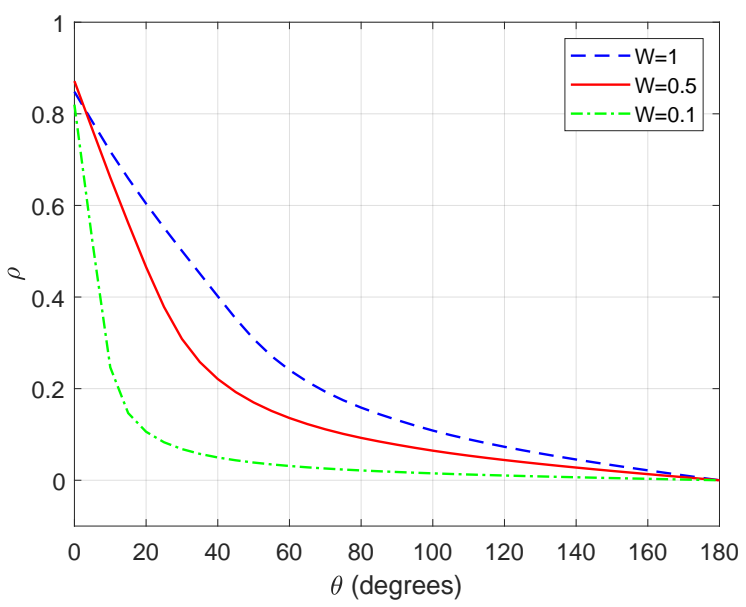

(a) Different values of blockage width $(W)$

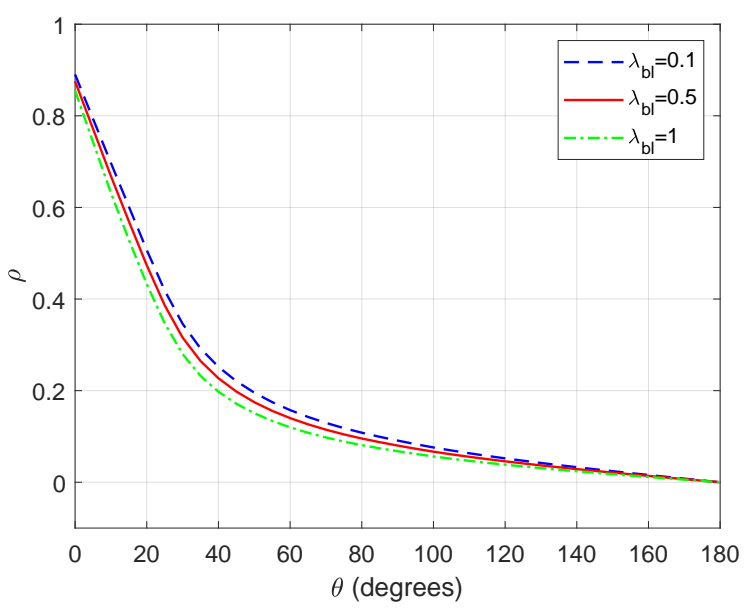

(b) Different values of blockage density $\left(\lambda_{b l}\right)$

Figure 4.3: The correlation coefficient $(\rho)$ versus the angular separation $(\theta)$ between $X_{1}$ and $X_{2}$.

the example, the distances from the source transmitter to the two base stations are fixed at $R_{1}=1.2$ and $R_{2}=1.5$ and the base station density is $\lambda_{b s}=0.3$. In Fig. 4.3(a), we fixed the blockage density at $\lambda_{b l}=0.6$, and the blockage width $W$ is varied. In Fig. 4.3(b), $W=0.5$ and the value of $\lambda_{b l}$ is varied. Both figures show that $\rho$ decreases with increasing $\theta$. This is because the area $v$ gets smaller as $\theta$ increases. As $\theta$ approaches 180 degrees, $v$ approaches zero, and the correlation is minimized. The figures show that correlation is more dramatic when $W$ is large, since a single large blockage is likely to simultaneously block both base stations, and when $\lambda_{b l}$ is small, which corresponds to the case that there are fewer blockages.

Fig. 4.4 shows the empirical cumulative distribution function (CDF) of $p_{L O S}$ over 1000 network realizations for first- and second-order macrodiversity, both with and without considering blockage correlation. The distributions are computed by fixing the value of $W=0.8$ and using two different values of the average number of blockages per base station $\left(\lambda_{b l} / \lambda_{b s}\right)$. The CDF of $p_{L O S}$ quantifies the likelihood that the $p_{L O S}$ is below some value. The figure shows the probability that $p_{L O S}$ is below some value increases significantly when the number of blockages per base station is high. The effect of correlated blocking is more pronounced when there are fewer blockages per base station. The macrodiversity gain is the improvement in performance for $N=2$ as compared to $N=1$, in the figure the macrodiversity gain is higher when the number of blockages per base station is lower even though the amount of 


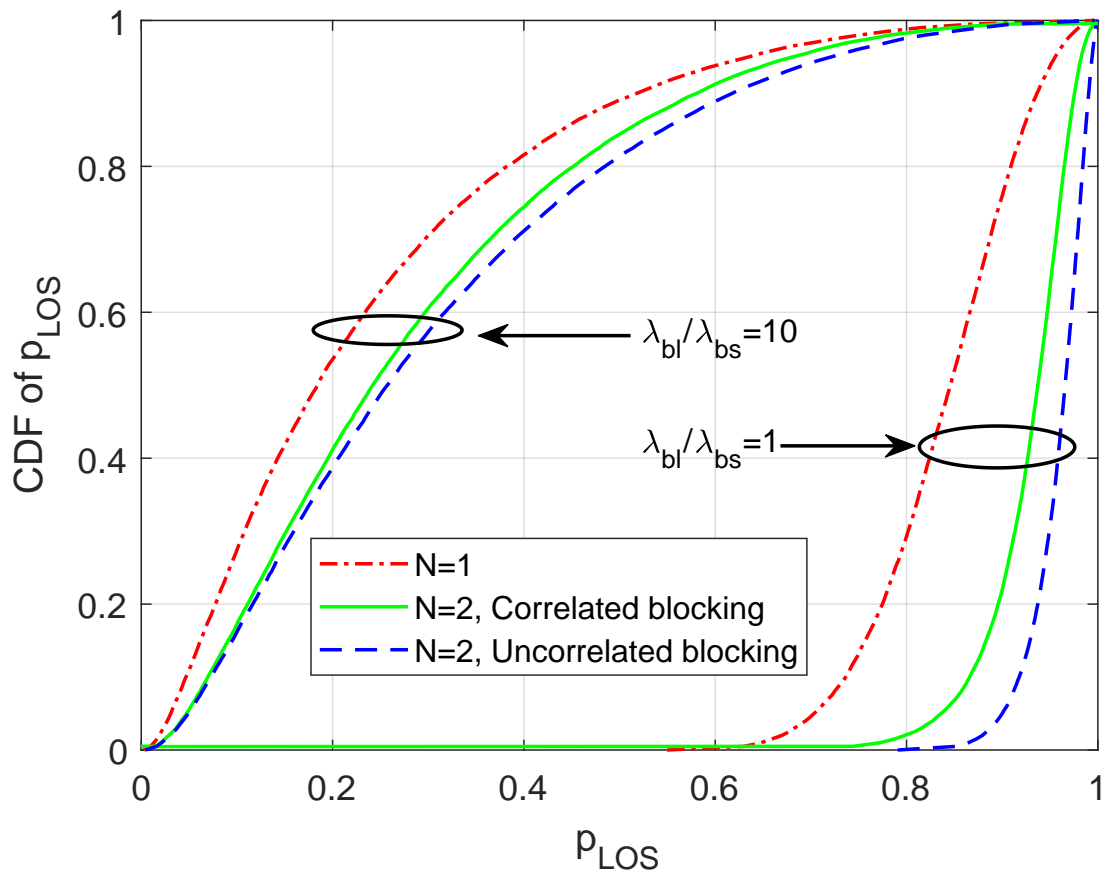

Figure 4.4: The empirical CDF of $p_{L O S}$ over 1000 network realizations when $N=1,2$, with and without considering blockage correlation at fixed blockage width $W=0.8$.

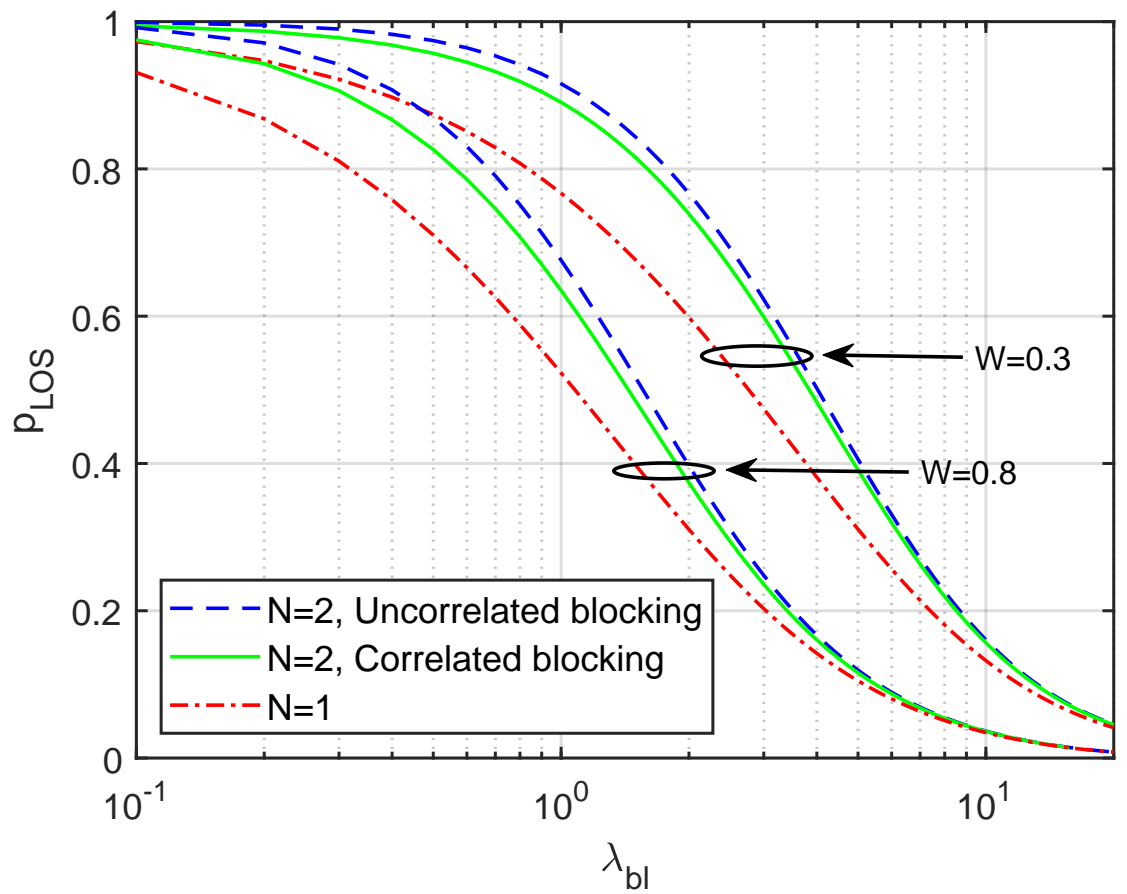

Figure 4.5: The variation of the spatially averaged $p_{L O S}$ over 1000 network realizations with respect to blockage density $\lambda_{b l}$ when $N=1,2$, with and without considering blockage correlation at fixed base station density $\lambda_{b s}=0.3$. 
reduction in gain due to correlation is higher when $\lambda_{b l} / \lambda_{b s}$ is lower.

Fig. 4.5 shows the variation of $p_{L O S}$ when averaged over 1000 network realizations. In this figure, $1000 p_{L O S}$ values is found for different 1000 network realization, then the averaged $p_{L O S}$ is calculated for different values of blockage density $\lambda_{b l}$. The derivation of the distances for each network realization can be found in Appendix B. The plot shows average $p_{L O S}$ as a function of $\lambda_{b l}$ while keeping base station density $\lambda_{b s}$ fixed at 0.3 . The spatially averaged $p_{L O S}$ is computed for two different values of blockage width $W$. Compared to the case of no diversity (when $N=1$ ), the second-order macrodiversity can significantly increase $p_{L O S}$. However, $p_{L O S}$ decreases when blockage size or blockage density is higher. Moreover, correlated blocking reduces the $p_{L O S}$ compared to independent blocking, and larger blockages increase the correlation, since a single large blockage is likely to simultaneously block both base stations. Comparing the two pairs of correlated/uncorrelated blocking curves, the correlation is more dramatic when $\lambda_{b l}$ is low, since at low $\lambda_{b l}$ both base stations are typically blocked by the same blockage (located in area $v$ ).

\subsection{SNR Distribution}

In this section, we consider the distribution of the SNR. Macrodiversity can be achieved by using either diversity combining, where the signals from the multiple base stations are maximum ratio combined, or selection combining, where only the signal with the strongest SNR is used. For $n^{\text {th }}$-order macrodiversity, the SNR with diversity combining is 56

$$
\mathrm{SNR}=\mathrm{SNR}_{0} \underbrace{\sum_{i=1}^{n}\left(1-B_{i}\right) \Omega_{i}}_{Z}
$$

where $\Omega_{i}=R_{i}^{-\alpha}$ is the power gain between the source transmitter $Y_{0}$ to the $i^{\text {th }}$ base station and $\mathrm{SNR}_{0}$ is the SNR of an unblocked reference link of unit distance. $B_{i}$ is used to indicate that the path between $Y_{0}$ and $X_{i}$ is blocked, and thus when $B_{i}=1, \Omega_{i}$ does not factor into the SNR.

The CDF of SNR, $F_{\mathrm{SNR}}(\beta)$, quantifies the likelihood that the combined SNR at the closest $n$ base stations is below some threshold $\beta$. If $\beta$ is interpreted as the minimum 
acceptable SNR required to achieve reliable communications, then $F_{\mathrm{SNR}}(\beta)$ is the outage probability of the system $P_{o}(\beta)=F_{\mathrm{SNR}}(\beta)$. The coverage probability is the complimentary $\mathrm{CDF}, P_{c}(\beta)=1-F_{\mathrm{SNR}}(\beta)$ and is the likelihood that the SNR is sufficiently high to provide coverage. The rate distribution can be found by linking the threshold $\beta$ to the transmission rate, for instance by using the appropriate expression for channel capacity.

The CDF of SNR evaluated at threshold $\beta$ is as follows:

$$
F_{\mathrm{SNR}}(\beta)=P[\mathrm{SNR} \leq \beta]=P\left[Z \leq \frac{\beta}{\mathrm{SNR}_{0}}\right]=F_{Z}\left(\frac{\beta}{\mathrm{SNR}_{0}}\right) .
$$

The discrete variable $Z$ represents the sum of the unblocked signals. To find the CDF of $Z$ we need to find the probability of each value of $Z$, which is found as follows for secondorder macrodiversity. The probability that $Z=0$ can be found by noting that $Z=0$ when both $X_{1}$ and $X_{2}$ are blocked. From (4.1), this is

$$
p_{Z}(0)=p_{B_{1}, B_{2}}(1,1)=p_{1} p_{2}+\rho h .
$$

The probability that $Z=\Omega_{i}, i \in\{1,2\}$ can be found by noting that $Z=\Omega_{i}$ when only $X_{i}$ is LOS. From 4.1), this is

$$
\begin{aligned}
& p_{Z}\left(\Omega_{1}\right)=p_{B_{1}, B_{2}}(0,1)=q_{1} p_{2}-\rho h . \\
& p_{Z}\left(\Omega_{2}\right)=p_{B_{1}, B_{2}}(1,0)=p_{1} q_{2}-\rho h .
\end{aligned}
$$

Finally, by noting that $Z=\Omega_{1}+\Omega_{2}$ when both $X_{1}$ and $X_{2}$ are LOS leads to

$$
p_{Z}\left(\Omega_{1}+\Omega_{2}\right)=p_{B_{1}, B_{2}}(0,0)=q_{1} q_{2}+\rho h .
$$

From 4.11) to 4.14, the CDF of $Z$ is found to be:

$$
F_{Z}(z)= \begin{cases}0 & \text { for } z<0 \\ p_{1} p_{2}+\rho h & \text { for } 0 \leq z<\Omega_{2} \\ p_{1} & \text { for } \Omega_{2} \leq z<\Omega_{1} \\ p_{1}+q_{1} p_{2}-\rho h & \text { for } \Omega_{1} \leq z<\Omega_{1}+\Omega_{2} \\ 1 & \text { for } z \geq \Omega_{1}+\Omega_{2} .\end{cases}
$$




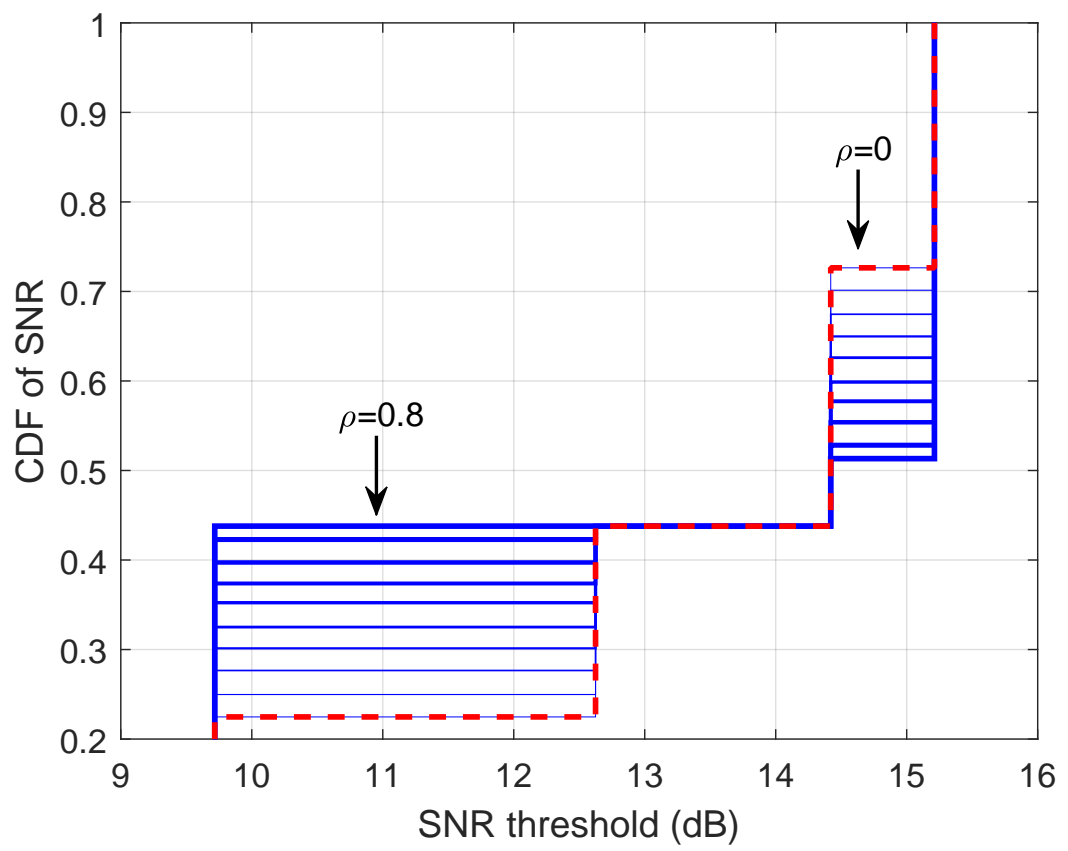

Figure 4.6: The CDF of the $\operatorname{SNR} F_{\text {SINR }}(\beta)$ using diversity combining for fixed location of $X_{1}$ and $X_{2}$ for different values of $\rho$. The dashed red line shows the CDF when $\rho=0$, and the solid blue lines correspond to positive values of $\rho$ in increments of 0.1 .

Next, in the case of selection combining, the SNR is 56

$$
\mathrm{SNR}=\mathrm{SNR}_{0} \underbrace{\max \left[\left(1-B_{1}\right) \Omega_{1},\left(1-B_{2}\right) \Omega_{2}, \ldots,\left(1-B_{n}\right) \Omega_{n}\right]}_{Z}
$$

and its CDF, from 4.11 to 4.13 is found for second-order macrodiversity to be:

$$
F_{Z}(z)= \begin{cases}0 & \text { for } z<0 \\ p_{1} p_{2}+\rho h & \text { for } 0 \leq z<\Omega_{2} \\ p_{1} & \text { for } \Omega_{2} \leq z<\Omega_{1} \\ 1 & \text { for } z \geq \Omega_{1} .\end{cases}
$$

Fig. 4.6 is an example showing the effect that the value of the correlation coefficient $\rho$ has upon the CDF of SNR. The curves were computed by placing the base stations at distances $R_{1}=2$ and $R_{2}=5$, and fixing the values of $\alpha=2$ and $\mathrm{SNR}_{0}=15 \mathrm{~dB}$. The values of $q_{i}$ and $p_{i}$ were computed using (4.3) and (4.4) respectively, by assuming $W=0.6, \lambda_{b l}=0.3$. The CDF is found assuming values of $\rho$ between $\rho=0$ to $\rho=0.8$ in increments of 0.1 ; the 


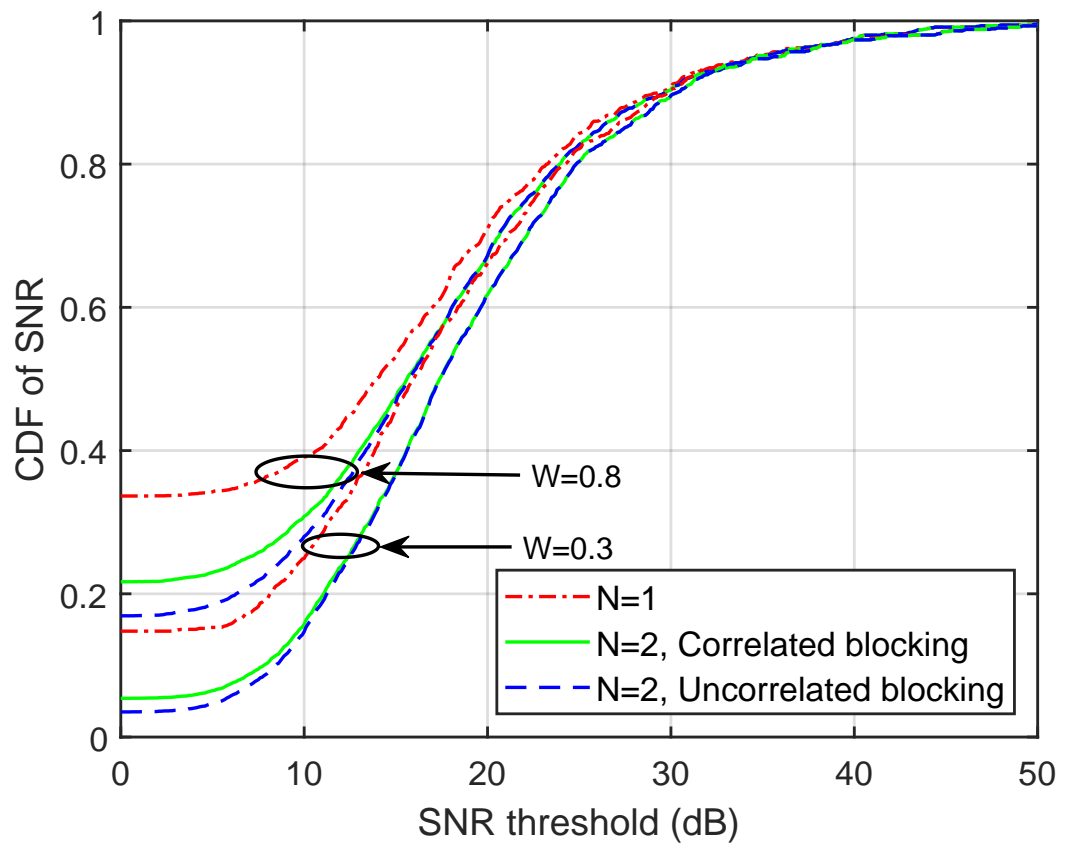

Figure 4.7: The distribution of SNR over 1000 network realizations when $N=1,2$ using diversity combining, with and without considering blockage correlation at fixed values of blockage density $\lambda_{b l}=0.6$ and base station density $\lambda_{b s}=0.3$.

value of $\rho$ can be adjusted by varying the angle $\theta$ between the two base stations. The dashed red line represents the case that $\rho=0$, corresponding to uncorrelated blocking. The solid blue lines correspond to positive values of $\rho$ in increments of 0.1 , where the thinnest line corresponds to $\rho=0.1$ and the thickest line corresponds to $\rho=0.8$.

Fig. 4.6 shows a first step up at $9.7 \mathrm{~dB}$, and the increment of the step is equal to the probability that both base stations are NLOS. The magnitude of the step gets larger as the blocking is more correlated, because correlation increases the chance that both base stations are NLOS (i.e., $\left.p_{B_{1}, B_{2}}(1,1)\right)$. The next step up occurs at $12.7 \mathrm{~dB}$, which is the SNR when just one of the two closest base stations is blocked, which in this case is the closest base station $X_{1}$. The next step at $14.5 \mathrm{~dB}$ represents the case when only $X_{2}$ is blocked, The magnitude of the two jumps is equal to the probability that only the corresponding one base station is LOS, and this magnitude decreases with positive correlation, because if one base station is LOS the other one is NLOS. Finally, there is a step at $15.2 \mathrm{~dB}$, which corresponds to the case that both base stations are $\operatorname{LOS}$. Notice that when $\rho=0.8$, the two middle steps merge. This is because for such a high value of, it is impossible for just one base station to 


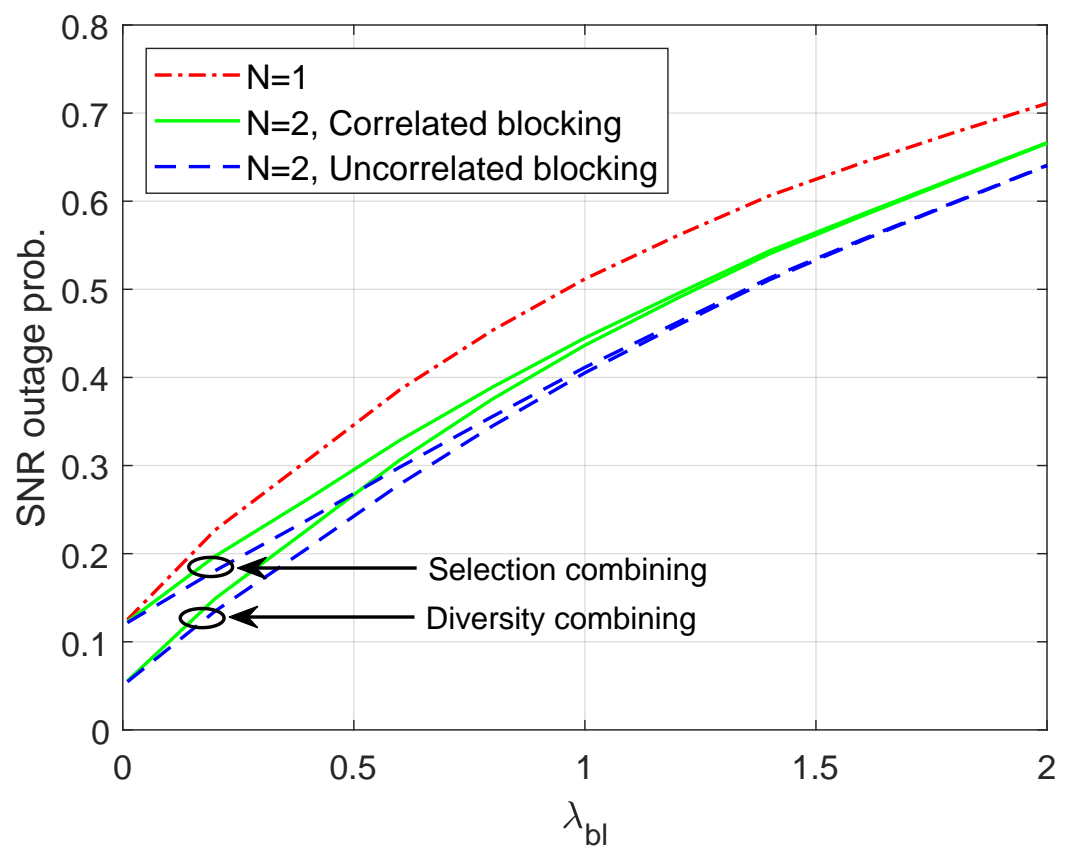

Figure 4.8: The SNR outage probability at threshold $\beta=10 \mathrm{~dB}$ with respect to $\lambda_{b l}$ when $N=1,2$, with and without considering blockage correlation at fixed values of blockage density $\lambda_{b s}=0.3$ and blockage width $W=0.8$.

be blocked, and most likely that both base stations are blocked, so the curve goes directly from $\mathrm{SNR}=9.7 \mathrm{~dB}$ to $\mathrm{SNR}=15.2 \mathrm{~dB}$.

Fig. 4.7 shows the CDF of SNR over 1000 network realizations for diversity combining and two different values of $W$ when $\lambda_{b s}=0.4$ and $\lambda_{b l}=0.6$. In addition, $\mathrm{SNR}_{0}$ and the path loss $\alpha$ are fixed at $15 \mathrm{~dB}$ and 3 respectively for the remaining figures in this chapter. It can be observed that the CDF increases when blockage size is larger. Compared to the case when $N=1$, the use of second-order macrodiversity decreases the SNR distribution. When compared to uncorrelated blocking, correlation decreases the gain of macrodiversity for certain regions of the plot, particularly at low values of SNR threshold, corresponding to the case when both base stations are blocked. Similar to $p_{L O S}$, the correlation increases with blockage size. However, the macrodiversity gain is slightly higher when blockage width $W$ is smaller.

Fig. 4.8 shows the effect of combining scheme and $\lambda_{b l}$ on SNR outage probability at threshold $\beta=10 \mathrm{~dB}$. As shown in the figure, the outage probability increases when $\lambda_{b l}$ increases in all of the given scenarios. When $\lambda_{b l}=0$, first- and second-order selection 
combining perform identically. This is because $X_{1}$ is never blocked. However, as $\lambda_{b l}$ increases, the gain of both selection combining and diversity combining increase. At high $\lambda_{b l}$ the combining scheme is less important, in which case the paths to $X_{1}$ and $X_{2}$ are always blocked regardless of the chosen combining scheme. The reduction in gain due to correlation is slightly higher when using selection combining. From Equation 4.17 this is because the step when both base stations are blocked is wider compared to diversity combining case.

\subsection{Validation with Real Data}

To validate our framework, we consider a region of West Virginia University campus as shown in Fig. 4.9 with base stations locations drawn from a PPP and a randomly placed user. The exterior walls of the buildings highlighted in red color are considered to be the blockages. The equivalent parameters for the statistical analysis introduced by this chapter are obtained by calculating the number of buildings, the area of each building, and the total area of the region. The average blockage width $(\mathrm{W})$ is found from the areas of the individual buildings $\left(A_{i}\right)$, such that the width of each blockage $W_{i}=2 \sqrt{A_{i} / \pi}$, while the blockage density is found as the the number of buildings divided by the total region area.

Fig. 4.10 shows the empirical CDF of SNR over 1000 network realizations computed using our statistical analysis and computed using the actual data. The total region area is found to be $335720 \mathrm{~m}^{2}$, the number of buildings is 49 , the average building width is $W=33$ $\mathrm{m}, \lambda_{b l}$ is the ratio of number of buildings to the total area, and $\lambda_{b s}=3 \lambda_{b l}$. We limited the environment to be outdoor by allowing the base stations and user to only be located outside buildings. It can be observed that the analysis approximates the performance in the real scenario very well. Compared to the curves representing the analysis when $N=2$, it is clear that the real data model when $N=2$ is closer to the case when considering correlated blocking compared to the case assuming independent blocking. This is because one building can simultaneously block more than one base station. In the actual region, the blockages have different sizes and orientations, this is in contrast with our model, which assumes a constant blockage size and orientation. Due to these differences, there is a small different between the statistical model and the real data based model as shown in the figure. 


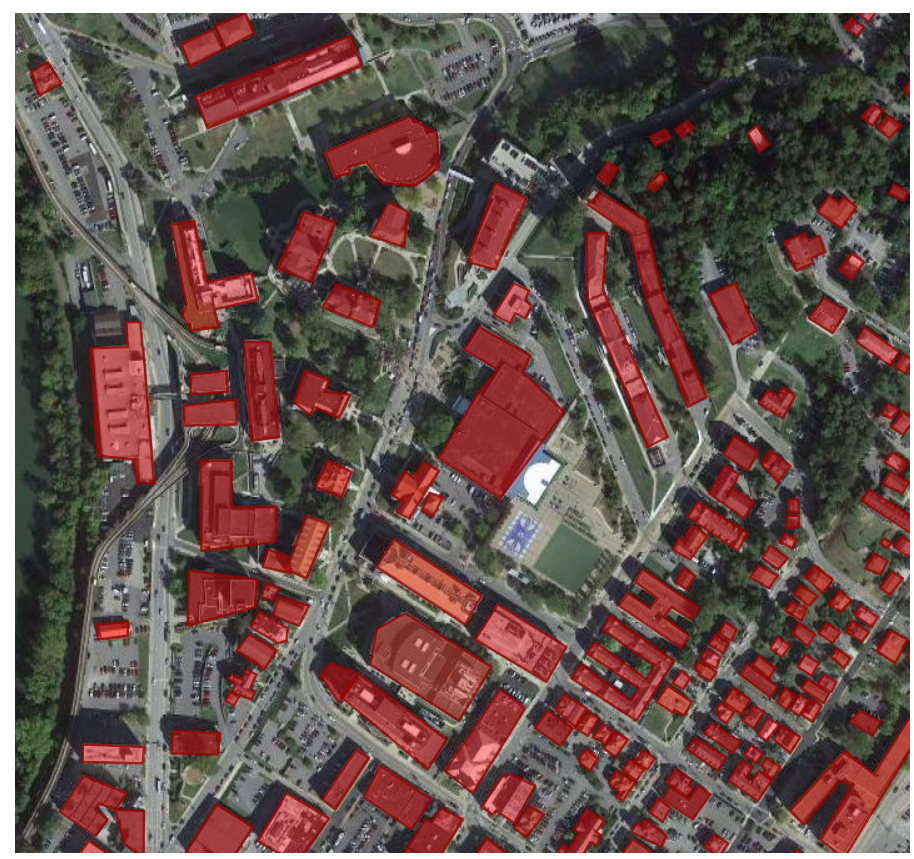

Figure 4.9: Map of WVU downtown campus. The red-highlighted buildings are the blockages, and the base stations and user are randomly placed over the region.

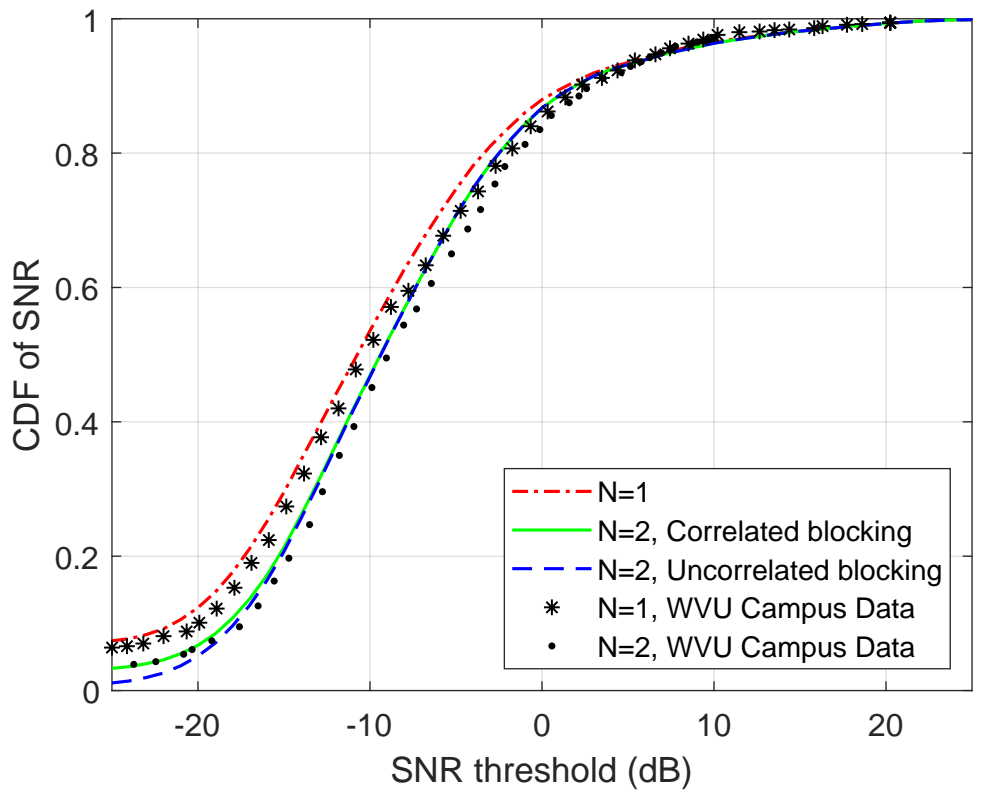

Figure 4.10: The distribution of SNR over 1000 network realizations when $N=1,2$ using diversity combining, plotted using the real data model and the analytical model. 


\subsection{SINR Outage Analysis}

Thus far, we have not assumed any interfering transmitters in the system. In practice, the received signal is also affected by the sum interference. The goal of this section is to formulate the CDF of SINR for second-order macrodiversity. SINR for first-order macrodeiversity along with blockage correlation between interferers has been considered in 22]. In this section, we assume each neighboring cell has a single interfering mobile, which is located uniformly within a disk of radius $r$ around the base station. Assuming a perfect packing of cells, $r=\left(\lambda_{b s} \pi\right)^{-1 / 2}$, which is the average cell radius. We explicitly consider the interference from the $M$ closest neighboring cells. The interference from more distant cells is considered to be part of the thermal noise. Let $Y_{j}$ for $j=1,2, . ., M$ indicate the interfering transmitters and their locations. Recall that $j=0$ indicates the source transmitter $Y_{0}$. The distance from the $j^{\text {th }}$ transmitter to the $i^{\text {th }}$ base station is denoted by $R_{i, j}$.

To calculate SINR and its distribution, we first define a matrix $\mathbf{B}$ which indicates the blocking state of the paths from $Y_{j}$ for $j=0,2, . ., M$ to $X_{i}$ for $i=1,2$. B is a Bernoulli Matrix of size 2 by $(M+1)$ elements. Each column in $\mathbf{B}$ contain elements $B_{1, j}$ and $B_{2, j}$ which indicate the blocking states of the paths from $Y_{j}$ to $X_{1}$ and $X_{2}$ respectively; i.e, the first column in $\mathbf{B}$ contains the pair of Bernoulli random variables $B_{1,0}$ and $B_{2,0}$ that indicates the blocking state of the paths from $Y_{0}$ to $X_{i}$ for $i=1,2$. There are $(M+1)$ pairs of Bernoulli random variables, and each pair is correlated with correlation coefficient $\rho_{j}$. Because the $2(M+1)$ elements of $\mathbf{B}$ are binary, there are $2^{2(M+1)}$ possible combinations of $\mathbf{B}$. However, it is possible for different realizations of $\mathbf{B}$ to correspond to the same value of SINR. For example, when $X_{1}$ and $X_{2}$ are both blocked from $Y_{0}$, the SINR will be the same value regardless of the blocking states of the interfering transmitters. Define $\mathbf{B}^{(n)}$ for $n=1,2, \ldots, 2^{2(M+1)}$ to be the $n^{\text {th }}$ such combination of B. Similar to Section 4.3 , let $p_{B_{1, j}, B_{2, j}}\left(b_{1, j}^{(n)}, b_{2, j}^{(n)}\right)$ be the joint probability of $B_{1, j}$ and $B_{2, j}$ which are the elements of the $j^{t h}$ column of $\mathbf{B}^{(n)}$. The probability of $\mathbf{B}^{(n)}$ is given by

$$
P\left(B^{(n)}\right)=\prod_{j=0}^{M} p_{B_{1, j}, B_{2, j}}\left(b_{1, j}^{(n)}, b_{2, j}^{(n)}\right)
$$


The SINR of a given realization $\mathbf{B}^{(n)}$ at base station $X_{i}$ is given by

$$
\operatorname{SINR}_{\mathrm{i}}^{(\mathrm{n})}=\frac{\left(1-B_{i, 0}^{(n)}\right) \Omega_{i, 0}}{\mathrm{SNR}_{0}^{-1}+\sum_{j=1}^{M}\left(1-B_{i, j}^{(n)}\right) \Omega_{i, j}}
$$

where $\Omega_{i, j}=R_{i, j}^{-\alpha}$ is the path gain from the $j^{\text {th }}$ transmitter at the $i^{\text {th }}$ base station. The SINR of the combined signal considering selective combining is expressed as

$$
\operatorname{SINR}^{(n)}=\max \left(\operatorname{SINR}_{1}^{(\mathrm{n})}, \operatorname{SINR}_{2}^{(\mathrm{n})}\right)
$$

When considering diversity combining 4.20 changes to

$$
\operatorname{SINR}^{(n)} \leq \operatorname{SINR}_{1}^{(\mathrm{n})}+\operatorname{SINR}_{2}^{(\mathrm{n})}
$$

As described in [57], correlated interference tends to make the combined SINR less than the sum of the individual SINRs. The bound in 4.21 is satisfied with equality when the interference is independent at the two base stations.

To generalize the formula for any realization, there is a particular SINR(n) associated with each $\mathbf{B}^{(n)}$. However, as referenced above, multiple realizations of $\mathbf{B}^{(n)}$ may result in the same SINR. Let SINR ${ }^{(\mathrm{k})}$ be the $k^{\text {th }}$ realization of SINR. Its probability is

$$
P\left(\operatorname{SINR}^{(k)}\right)=\sum_{n: \operatorname{SINR}=\operatorname{SINR}^{(k)}} P\left(B^{(n)}\right)
$$

Fig. 4.11 shows the distributions of SINR for $M=5$ and $M=0$ (which is SNR) at fixed values of $\lambda_{b s}=0.3, \lambda_{b l}=0.6$, and $W=0.6$. The distributions are computed for firstand second-order macrodiversity. It can be observed that macrodiversity gain is reduced when interference is considered. This is because of the increase in sum interference due to macrodiversity, which implies that $p_{L O S}$ alone as in 44] may not be sufficient to predict the performance of the system especially when there are many interfering transmitters. Study of higher order macrodiversity to identify the minimum order of macrodiversity to achieve a desired level of performance in the presence of interference is left for future work.

Fig. 4.12 shows the variation of SINR outage probability with respect to the number of interfering transmitters $M$. The curves are computed for low and high values of $\lambda_{b l}$, 


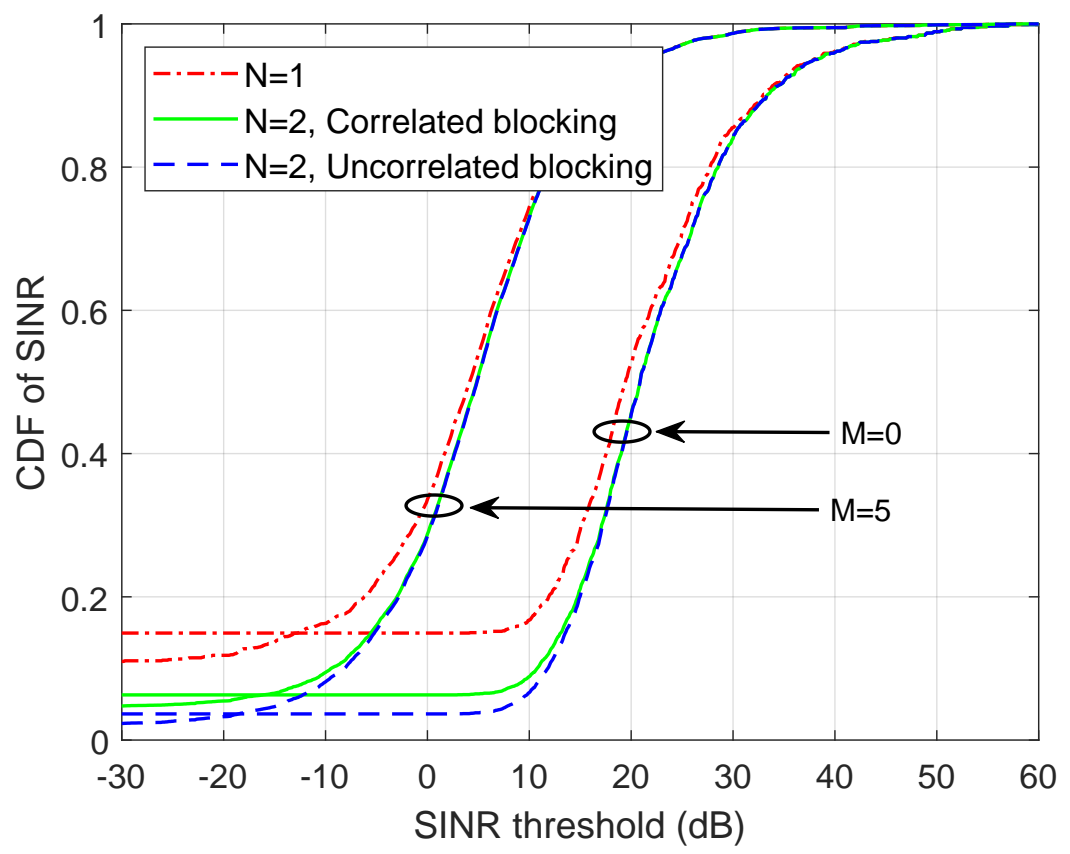

Figure 4.11: The distribution of SINR over 1000 network realizations using diversity combining for different values of number of interfering transmitters. The curves are computed when $N=1,2$, with and without considering blockage correlation, at fixed values of $\lambda_{b s}=0.3$, $\lambda_{b l}=0.6$, and $W=0.6$.

while keeping $\lambda_{b s}$ and $W$ fixed at 0.8 and 0.6 respectively. It can be seen that the outage probability increases when $M$ increases. Due to the fact that interference tends to also be blocked, unlike SNR and $p_{L O S}$, increasing the $\lambda_{b l}$ decreases the outage probability. Similar to Fig. 4.11, the macrodiversity gain decreases significantly when $M$ increases. It can be seen that $N=2$ curves reaches the case when $N=1$ for $M=6$. Compared to uncorrelated blocking, the curves considering correlated blocking matches the uncorrelated cases for high value of $M$, since the interfering transmitters are placed farther than source transmitter and their overlapping area is less dominant.

Fig. 4.13 shows the variation of the outage probability with respect to the order of macrodiversity. The curves are computed for $M=1, M=3$, and $M=10$. The results shows that as the macrodiversity order the outage decrease and as the number of interfering transmitters increases the outage decrease. However, the results also show that the amount of decrease in the outage is getting smaller as $\mathrm{M}$ increases. For example when $M=1$ the outage decrease faster than when $M=10$ and when $N=1$ the gab between $M=1$ 


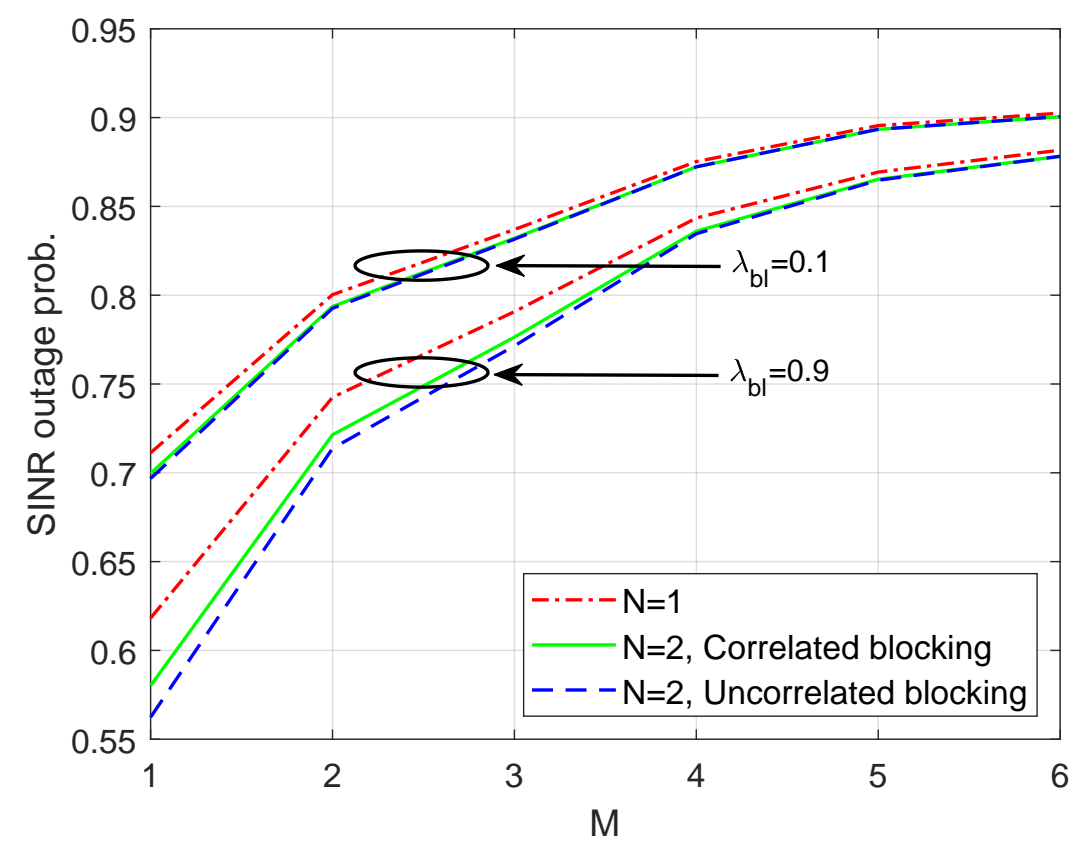

Figure 4.12: The outage probability of SINR at threshold $\beta=15 \mathrm{~dB}$ versus the number of interfering transmitters $(M)$, when $N=1,2$, with and without considering blockage correlation, at fixed values of $\lambda_{b s}=0.8$ and $W=0.6$.

and $M=10$ is very small, but when increasing $N$ to 6 the gab gets much larger. From this results we can identify the minimum macrodiversity order required to achieve desired macrodiversity gain that minimizes the outage in the presence of interference.

\subsection{Conclusion}

We have proposed a framework to analyze the second-order macrodiversity gain for mmWave cellular system in the presence of correlated blocking. Correlation is an important consideration for macrodiversity because a single blockage can block multiple base stations, especially if the blockage is sufficiently large and the base stations sufficiently close. The assumption of independent blocking leads to an incorrect evaluation of macrodiversity gain of the system. By using the methodology in this chapter, the correlation between two base stations is found and factored into the analysis. The chapter considered the distributions of LOS probability, SNR, and, when there is interference, the SINR. The framework was confirmed by comparing the analysis to a real data model. We show that correlated blocking 


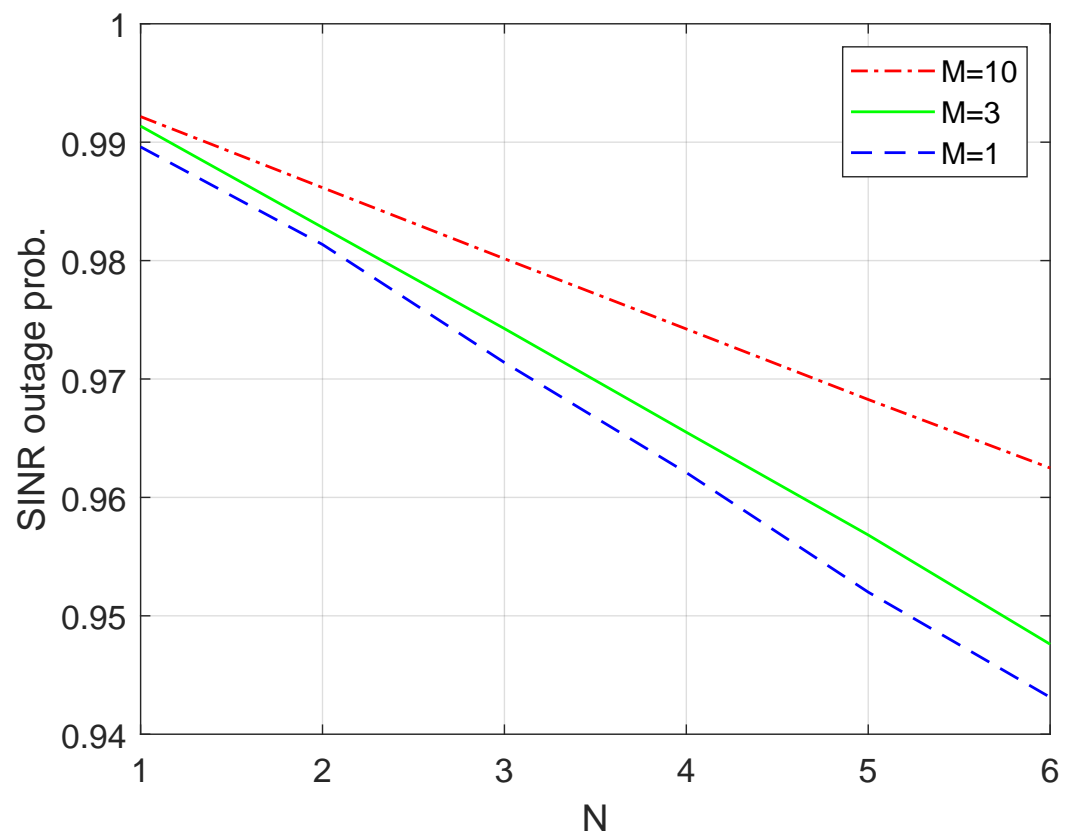

Figure 4.13: The outage probability of SINR at threshold $\beta=10 \mathrm{~dB}$ versus the macrodiveristy order $(N)$, when $M=1,3,10$, at fixed values of $\lambda_{b s}=0.8$ and $W=0.6$.

decreases the macrodiversity gain. We also study the impact of blockage size and blockage density. We show that blockage can be both a blessing and a curse. On the one hand, the signal from the source transmitter could be blocked, and on the other hand, interfering signals tend to also be blocked, which leads to a completely different effect on macrodiversity gains. 


\section{Chapter 5}

\section{Optimization of a Millimeter-Wave UAV-to-Ground Network in Urban Deployments}

\section{$5.1 \quad$ Introduction}

Networks based on Unmanned Aerial Vehicles (UAVs) can provide instant infrastructure for services such as surveillance, broadband access, networked control, and automation 17, 18, 20, 21]. For such networks, the use of millimeter-wave (mmWave) frequencies is an attractive option thanks to the high available bandwidth coupled with spatial isolation due to beamforming [2,19]. In urban areas, among the most challenging of environments, buildings pose a major source of blockage to the line-of-sight (LoS) propagation path [7,23]. While reflections are possible at mmWave, the desire for consistent and reliable connectivity places a strong preference on the existence of an unblocked LoS path.

In this chapter, we consider the performance and optimization of a network of randomly deployed UAVs in a typical urban environment. The analysis leverages tools from stochastic geometry to model both the network realization (i.e., the UAV locations) and the city environment (i.e., the heights and locations of buildings). The key performance metric developed here is the connectivity probability, which characterizes the likelihood that a typical user of the network (assumed to be a vehicle located on a city street) is able to connect to a LoS 
UAV within some maximum communication range.

Stochastic geometry has previously been used to study the LoS probability in twodimensional mmWave networks $13,14,22,30,46$, where the locations of blockages are drawn from an appropriate random process. Those works are not directed towards urban environments and do not account for the grid-like arrangements of city streets or the variable heights of buildings, not all of which will be tall enough to block the LoS. The grid-like pattern of city streets is considered in [58], which uses a Manhattan Poisson Line Process (MPLP) to model patterns of perpendicular streets. Building upon that work, [59] also uses a MPLP to model the grid of streets, but it additionally accounts for random building heights. The analysis of [59] derives the LoS probability to a single UAV as well as its spatial average (called the area LoS probability) when the UAV is uniformly distributed at fixed height within a circular cell.

Similar to [58, 59], this work uses a MPLP to account for the randomness of the city streets, and hence the random locations of buildings contained within blocks. Like [59], the random heights of buildings are considered, but unlike [59], the work in this chapter is not limited to a single UAV. Hence, a main technical contribution of this chapter is that it extends the results in [59] to the case of a network of multiple UAVs that are randomly scattered within the operational area. This extension allows for two key applications: (1) Quantification of the tradeoff between the UAV density (average number of UAVs per $\mathrm{km}^{2}$ ) and the network performance, which identifies the minimum number of UAVs required to cover an area at a desired performance level, and (2) Identification of the optimal UAV height for a given deployment scenario (city type) [60].

After introducing the system model in Section II, the chapter provides an analysis in Section III. The analysis begins by defining the connectivity probability, first considering the probability when it is conditioned on the placement of the UAVs, then removing the conditioning to reveal the distribution of the connectivity; i.e., the probability that a network realization has a connectivity probability that is less than a threshold. By defining the outage probability to be the connectivity distribution evaluated at the minimum acceptable connectivity probability, we are able to characterize the tradeoff between the density of UAV and outage probability. Finally, for a given UAV density and type of city (e.g., urban, 


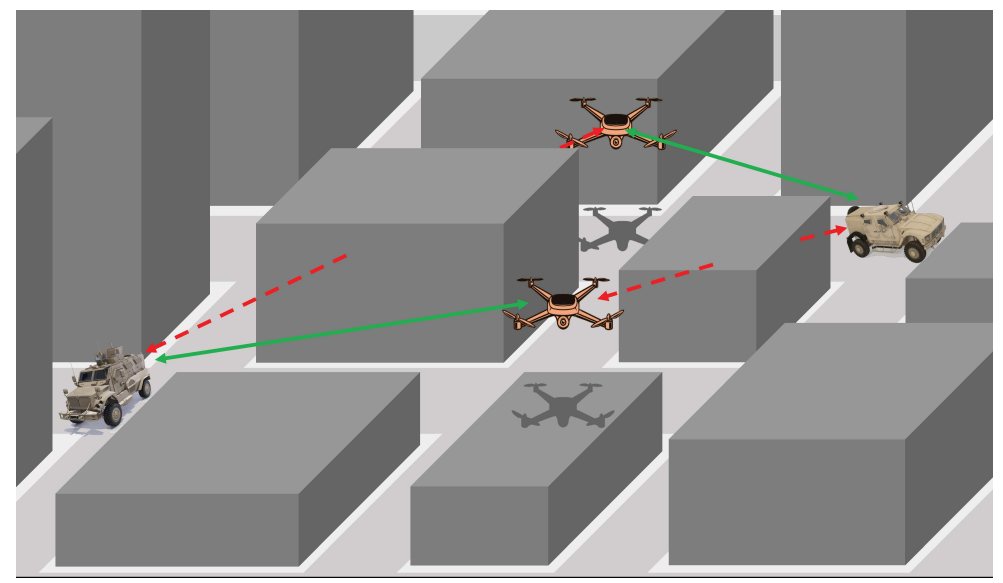

Figure 5.1: Example of 3D Urban aerial network consisting of UAVs, user vehicles, and buildings. Green solid lines indicate LoS links while red dashed lines indicate non-LoS. A shadow is shown directly below each UAV to identify its projection onto the ground plane. Street widths are exaggerated relative to building widths.

dense urban, suburban), we are able to optimize the UAV height by finding the value that minimizes the outage probability. Numerical results are provided in Section IV, and the chapter concludes with Section V.

\subsection{System Model}

As illustrated in Fig. 5.1, the network consists of UAVs in the air, users on the ground (typically located in vehicles), and blocks of buildings that serve as potential sources of blocking. The figure shows two UAVs and two user vehicles. A shadow is shown directly below each UAV to identify its projection onto the building or street below it. Each UAV is LOS to one of the UAVs, but its signal to the other UAV is blocked by a building.

Information communicated between the ground user and the UAVs is either backhauled to some central gateway when the destination is external to the network, or it is routed in the air to another UAV when the destination is internal to it. Backhauling and routing are outside the scope of this chapter, but rather we focus exclusively on the UAV-to-ground link.

The city is laid out as a grid with streets either going in the east-west direction (Xaxis) or the north-south direction (Y-axis). The locations of the streets are drawn from a MPLP, which is a pair of independent one-dimensional Poisson point processes (PPPs) of 
density $\lambda_{\mathbf{s}}$, one for each dimension along the ground plane. In each dimension, the interval between neighboring points corresponds to the width of one street and the length of one building block. The mean street width is $\mu_{\mathrm{s}}$ and the mean block length is $\mu_{\mathrm{b}}$. Accordingly, $\lambda_{\mathrm{s}}=1 /\left(\mu_{\mathrm{s}}+\mu_{\mathrm{b}}\right)$. It is assumed that each block contains at least one building, and if there are multiple buildings, they are all the same height. The height of a block is represented by the random variable $H_{B}$ with cumulative distribution function $F_{H_{B}}(h)$ and mean $\mu_{H}$. For ease of exposition, we assume that $H_{B}$ is uniformly distributed between a minimum height $H_{\min }$ and a maximum height $H_{\max }$, but other distributions such as exponential or Rayleigh can be considered (see [59]).

Let $Y$ denote a reference user and its location. Without loss of generality, assume that the user is located at the origin; i.e., $Y=0$. The user is in a vehicle with an antenna of height $h_{\mathrm{V}}$ and located either on a north-south street of width $w_{v}$, on an east-west street of width $w_{h}$, or at an intersection, in which case it is simultaneously on a north-south street and an east-west street. Accordingly, we consider two distinct cases: (1) The vehicle is at an intersection, or (2) The vehicle is not at an intersection. Note that under this model, the reference station is at a fixed location while the topology of the city (location of streets and heights of buildings) changes for each realization of the underlying random model. Clearly, this is not literally how a city behaves - the buildings and streets are in known locations and do not change frequently. However, for a large city with a homogeneous distribution of streets and buildings, this is equivalent to randomly dropping the station at a different location within the city during each realization of the model. Thus, this is an effective model for a moving or randomly placed user and enables a good characterization of the city as a whole.

We assume that the UAVs are randomly deployed and model their locations with a twodimensional homogeneous PPP with density $\lambda_{\mathrm{UAV}}$. The UAVs are all at a constant height (or altitude) $h_{\text {UAV }}$. As illustrated in Fig. 5.2, let $X_{i}$ for $\mathrm{i} \in \mathbb{Z}^{+}$denote the UAVs and their locations as projected onto the ground plane. Let $d_{i}=\left|X_{i}\right|$ be the distance from $Y$ to $X_{i}$. As shown in Fig. 5.2, $d_{i}$ is the distance from the user's vehicle to the projection of the UAV onto the ground (henceforth called the "2D distance"), while $r_{i}$ is the distance from the vehicle's antenna to the UAV (the "3D distance"). The azimuth angle of departure of the 


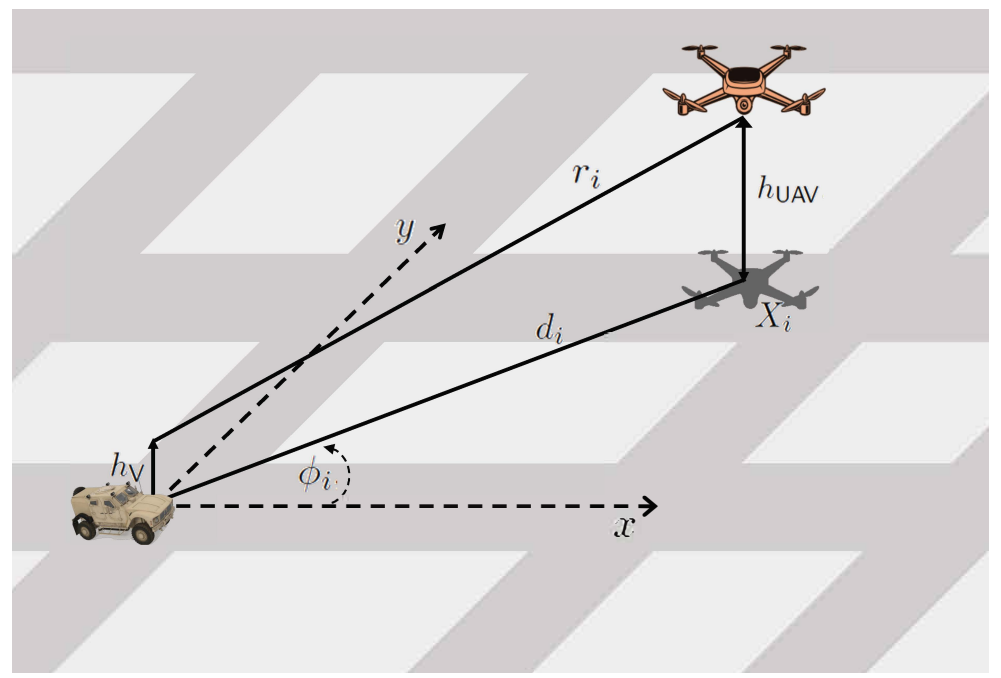

Figure 5.2: Coordinates associated with the $i^{\text {th }} \mathrm{UAV}$, including its location $X_{i}$ as projected onto the ground, the 2D distance $d_{i}$ from the vehicle to the projection, the azimuth angle $\phi_{i}$ relative to the positive $X$ axis, the height of UAV $h_{\mathrm{UAV}}$, the height of the vehicle antenna $h_{\mathrm{V}}$, and the $3 \mathrm{D}$ distance $r_{i}$.

UAV-vehicle path is denoted by $\phi_{i}$, which is uniform over $(0,2 \pi)$ since the UAVs are drawn from a homogenous PPP. The UAVs are indexed according to their distances to $Y$ such that $d_{1} \leq d_{2} \leq \ldots$

The user may connect to any unblocked UAV that is within a maximum 3D transmission range $r_{\max }$. The maximum range $r_{\max }$ accounts for physical-layer parameters including the transmit power, antenna gains, noise floor, fading margin, atmospheric effects, and transmission technology. To account for the UAVs being above the ground plane, the maximum 3D transmission range must be projected onto a maximum 2D transmission range $d_{\max }=\sqrt{r_{\max }^{2}-\left(h_{\mathrm{UAV}}-h_{\mathrm{V}}\right)^{2}}$. The user is considered to be connected if it has a LOS path to any UAV whose ground projection $X_{i}$ is within the disk of radius $d_{\max }$. Because the UAVs are drawn from a PPP, it follows that the number $N$ of UAVs within this disk is Poisson with mean $\lambda_{\mathrm{UAV}} \pi d_{\mathrm{max}}^{2}$. The set of all UAVs within this disk forms a set of prospective UAVs with which the user may communicate. Denote this set by $\mathcal{X}$; i.e., $\mathcal{X}=\left\{X_{1}, X_{2}, \ldots, X_{N}\right\}$. 


\subsection{LoS Distribution and Outage Probability}

The connectivity probability $p_{\mathrm{c}}$ is the probability that at least one UAV within the maximum transmission range has an unblocked LoS to the user. The connectivity probability depends on whether the user's vehicle is at an intersection or outside an intersection. Initially, assume that it is at an intersection (the alternative case will be considered later). The connectivity probability also depends on the UAV locations $\mathcal{X}$ as well as the realization of the city environment (i.e., the MPLP governing the street locations and the building heights). First, assume the UAVs locations $\mathcal{X}$ are fixed (this conditioning will be removed later). The corresponding conditional connectivity probability is denoted by $p_{\mathrm{c}}(\mathcal{X})$.

In an urban environment, there is likely to be at least some correlation in the blockage events, since a large building could potentially block many UAVs. However, for an initial analysis, we assume here that the UAVs are independently blocked. Extension of this analysis to correlated blocking is left for future study as we discuss in the Conclusion. Under the assumption of independent blocking and noting that the vehicle is unable to connect only when all UAVs within the maximum range are blocked (i.e., non-LoS), the conditional probability of coverage can be found as

$$
p_{\mathrm{c}}(\mathcal{X})=1-\prod_{i=1}^{N}\left(1-p_{\operatorname{LoS}}\left(X_{i}\right)\right)
$$

where $p_{\text {LoS }}\left(X_{i}\right)$ is the probability that the path between $Y$ and $X_{i}$ is LoS.

As in [59], the value of $p_{\text {LoS }}\left(X_{i}\right)$ may be found by noting that (1) The intersection containing the vehicle is at a known location (the origin), and hence the pair of perpendicular streets flowing through that intersection are at deterministic locations, and (2) The other streets are drawn from the MPLP, which comprises two independent PPPs, and hence the north-south and east-west streets are independent. These properties allow $p_{\text {LoS }}\left(X_{i}\right)$ to be decomposed as

$$
p_{\mathrm{LoS}}\left(X_{i}\right)=p_{\mathrm{LoS}}^{(0)}\left(X_{i}\right) p_{\mathrm{LoS}}^{(x)}\left(X_{i}\right) p_{\mathrm{LoS}}^{(y)}\left(X_{i}\right)
$$

where $p_{\text {LoS }}^{(0)}\left(X_{i}\right)$ is the probability that the link is not blocked by the building closest to the vehicle, which is at a deterministic location and on the same street as the vehicle, $p_{\text {LoS }}^{(x)}\left(X_{i}\right)$ 
is the probability that it is not blocked by a building side that is perpendicular to the $\mathrm{X}$-axis (governed by the first PPP), and $p_{\text {LoS }}^{(y)}\left(X_{i}\right)$ is the probability that it is not blocked by a side that is perpendicular to the Y-axis (governed by the second PPP).

Finding the first factor in 5.2 requires identifying a critical height $h_{i}^{(0)}$ for the building. If the building neighboring the intersection is taller than this height, then $X_{i}$ will be blocked by it. From the geometry of Fig. 5.2 (see also [59]), this height is

$$
h_{i}^{(0)}=\frac{\max \left(\frac{w_{\mathrm{v}}}{2}, \frac{w_{\mathrm{v}}}{2} \cot \phi_{i}\right)\left(h_{\mathrm{UAV}}-h_{\mathrm{V}}\right)}{d_{i} \cos \phi_{i}}+h_{\mathrm{V}} .
$$

It follows that the probability that the nearest building does not block the link is the CDF of the building height evaluated at the critical height

$$
p_{\mathrm{LoS}}^{(0)}\left(X_{i}\right)=F_{H_{B}}\left(h_{i}^{(0)}\right)
$$

The second and third factors of (5.2) require determining the likelihood that there is a building side in the corresponding direction that is sufficiently high to block the UAV. If building heights were ignored, then the probability can be found from the void probability of the corresponding PPP, which gives the probability that there are no points (building sides) in the desired interval. For a homogeneous PPP of intensity $\lambda$ and interval of length $\ell$, the void probability is $\exp (-\lambda \ell)$. However, when height is considered, the PPP is thinned such that any point whose corresponding building side has a height that is less than the critical height for that location must be removed. The result is a non-homogenous PPP whose density is a function of distance, and its void probability is found by integrating the density along the interval. Letting $Z \in\{X, Y\}$ indicate either the $\mathrm{X}$ or $\mathrm{Y}$ direction, the resulting void probability of the thinned PPP is

$$
p_{\mathrm{LoS}}^{(z)}\left(X_{i}\right)=\exp \left(-\int_{z_{\mathrm{a}}}^{z_{\mathrm{b}}} \lambda_{\mathrm{s}}^{(z)}(z) d z\right)
$$

where $\lambda_{\mathrm{s}}^{(z)}(z)$ is the non-homogenous density of the thinned PPP in the Z direction, while $z_{\mathrm{a}}$ and $z_{\mathrm{b}}$ are the limits of the integration (discussed below).

A point at coordinate $z$ will be removed if the corresponding building side at that coordinate is below a critical height for that coordinate, otherwise it will be retained. Since the 
probability that a point at coordinate $z$ will be retained is the complement of the CDF of the building height evaluated at that coordinate, the density of the thinned PPP becomes

$$
\lambda_{\mathrm{s}}^{(z)}(z)=\lambda_{\mathrm{s}}\left[1-F_{H_{B}}\left(h_{i}^{(z)}(z)\right)\right]
$$

where $h_{i}^{(z)}(z)$ is the critical height in the $\mathrm{Z}$ direction at coordinate $\mathrm{z}$, which can be found from the geometry of the problem.

In the $\mathrm{X}$ direction, the critical height is

$$
h_{i}^{(x)}(x)=\frac{x\left(h_{\mathrm{UAV}}-h_{\mathrm{V}}\right)+h_{\mathrm{V}} d_{i} \cos \phi_{i}}{d_{i} \cos \phi_{i}}
$$

while in the $\mathrm{Y}$ direction it is

$$
h_{i}^{(y)}(y)=\frac{y\left(h_{\mathrm{UAV}}-h_{\mathrm{V}}\right)+h_{\mathrm{V}} d_{i} \sin \phi_{i}}{d_{i} \sin \phi_{i}} .
$$

For the $\mathrm{X}$ direction, the limits of integration in (5.5) are

$$
\begin{aligned}
z_{\mathrm{a}} & =\max \left(\frac{w_{\mathrm{v}}}{2}, \frac{w_{\mathrm{v}}}{2} \cot \phi_{i}\right) \\
z_{\mathrm{b}} & =d_{i} \cos \phi_{i}
\end{aligned}
$$

while for the $\mathrm{Y}$ direction they are

$$
\begin{aligned}
z_{\mathrm{a}} & =\max \left(\frac{w_{\mathrm{h}}}{2}, \frac{w_{\mathrm{h}}}{2} \tan \phi_{i}\right) \\
z_{\mathrm{b}} & =d_{i} \sin \phi_{i} .
\end{aligned}
$$

Since the connectivity probability depends on whether or not the vehicle is at an intersection, we define $p_{\mathrm{csec}_{\mathrm{sec}}}(\mathcal{X})$ to be the conditional connectivity probability when the vehicle is at an intersection and $p_{\mathrm{cstr}}(\mathcal{X})$ to be the conditional connectivity probability when it is not. $p_{\mathrm{csec}_{\mathrm{sec}}}(\mathcal{X})$ is found using the methodology given above, while $p_{\mathrm{c}_{\mathrm{str}}}(\mathcal{X})$ is found by setting $w_{\mathrm{v}}=0$ or $w_{\mathrm{h}}=0$ (depending on whether the vehicle is located on a north-south street or an east-west street).

Because the UAVs are randomly located, the value of $p_{\mathrm{c}}(\mathcal{X})$ will vary from one network realization to the next. Hence $p_{\mathrm{c}}$ is itself a random variable, and the CDF of $p_{\mathrm{c}}$ can be described as

$$
F_{\mathrm{c}}(\gamma)=\mathbb{P}\left[p_{\mathrm{c}} \leq \gamma\right]
$$


Table 5.1: Parameters For the URban Grid Model

\begin{tabular}{|c|c|c|c|}
\hline Type & $\begin{array}{c}\text { Mean building } \\
\text { height, } \mu_{\mathrm{H}}\end{array}$ & $\begin{array}{c}\text { Mean side } \\
\text { width, } \mu_{\mathrm{b}}\end{array}$ & $\begin{array}{c}\text { Mean street } \\
\text { width, } \mu_{\mathrm{s}}\end{array}$ \\
\hline \hline Suburban & $10 \mathrm{~m}$ & $37 \mathrm{~m}$ & $10 \mathrm{~m}$ \\
Urban & $19 \mathrm{~m}$ & $45 \mathrm{~m}$ & $13 \mathrm{~m}$ \\
Dense urban & $25 \mathrm{~m}$ & $60 \mathrm{~m}$ & $20 \mathrm{~m}$ \\
\hline
\end{tabular}

The overall CDF is the average of the CDFs of $p_{\mathrm{csec}_{\mathrm{sec}}}$ and $p_{\mathrm{cstr}}$, where the probability of being in an intersection is $\mu_{\mathrm{s}} /\left(\mu_{\mathrm{s}}+\mu_{\mathrm{b}}\right)$. Equation (5.11) can be rewritten as

$$
\begin{aligned}
F_{\mathrm{c}}(\gamma)= & \left(\frac{\mu_{\mathrm{s}}}{\mu_{\mathrm{s}}+\mu_{\mathrm{b}}}\right) \mathbb{P}\left[p_{\mathrm{csec}_{\mathrm{s}}} \leq \gamma\right] \\
& +\left(1-\frac{\mu_{\mathrm{s}}}{\mu_{\mathrm{s}}+\mu_{\mathrm{b}}}\right) \mathbb{P}\left[p_{\mathrm{cstr}} \leq \gamma\right] .
\end{aligned}
$$

The CDF of $p_{\mathrm{c}}, F_{\mathrm{c}}(\gamma)$, quantifies the likelihood that the $p_{\mathrm{c}}$ is below some threshold $\gamma$. If $\gamma_{\text {th }}$ is interpreted as the minimum acceptable $p_{\mathrm{c}}$ required to achieve reliable communications, then $F_{\mathrm{c}}\left(\gamma_{\mathrm{th}}\right)$ is the outage probability of the system. The outage probability can be used to evaluate the tradeoff of using different UAV densities. In addition, it can be used to find the optimal UAV height for a given UAV density and city type. The optimization of UAV height may be expressed as

$$
\min _{h_{\text {UAV }}} F_{\mathrm{c}}\left(\gamma_{\text {th }}\right), \quad \text { s.t. } \quad 0<r_{\max }+h_{\mathrm{V}}<h_{\text {UAV }}
$$

While 5.13 could be solved using classical gradient descent approaches, a pragmatic approach to solving it is to simply generate the $p_{c}$ distribution for a range of UAV densities and heights, read the outage probability from those curves, and look for the height that minimizes the outage probabilty for each UAV density.

\section{$5.4 \quad$ Numerical Results}

In this section, we begin by illustrating the connectivity distribution through numerical examples. We then investigate the outage probability for a variety of city types, UAV densities, and UAV heights. Finally, we find the optimal UAV altitude that minimizes the outage probability for different city types as a function of UAV density. Three city types are considered: Suburban, urban, and dense urban. The parameters used for each of these 


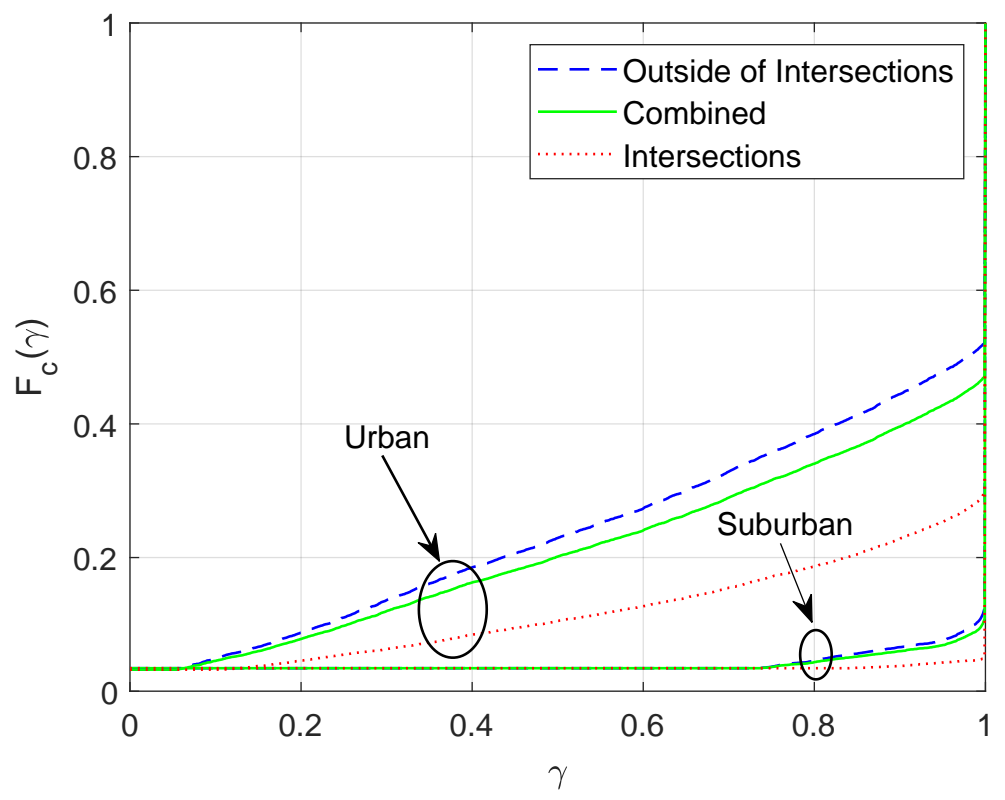

Figure 5.3: The connectivity distribution for urban and suburban deployments, considering two possible vehicle locations (at intersections or outside intersections) and the average across both locations. UAV density, UAV height, and maximum transmission range are fixed at $\lambda_{\text {UAV }}=20 / \mathrm{km}^{2}, h_{\text {UAV }}=100 \mathrm{~m}$, and $r_{\max }=250 \mathrm{~m}$.

city types is specified in Table I. When non-zero values are needed for $w_{\mathrm{h}}$ and $w_{\mathrm{v}}$, they are set to the value of $\mu_{\mathrm{s}}$ given in the table. The minimum and maximum building heights are $H_{\min }=\mu_{H} / 2$ and $H_{\max }=3 \mu_{H} / 2$, respectively. Throughout this section, the height of the vehicle's antenna is set to $h_{\mathrm{V}}=10 \mathrm{~m}$.

Because $p_{\mathrm{c}}$ depends on $\mathcal{X}$, its distribution $F_{\mathrm{c}}(\gamma)$ can be found by defining the equivalent event $\mathcal{X}^{\prime}=\left\{\mathcal{X}: p_{\mathrm{c}}(\mathcal{X}) \leq \gamma\right\}$ and then finding the probability that $\mathcal{X}$ is in $\mathcal{X}^{\prime}$; i.e., $F_{\mathrm{c}}(\gamma)=$ $\mathbb{P}\left(\mathcal{X} \in \mathcal{X}^{\prime}\right)$. However, this requires the inversion of the equation for $p_{c}(\mathcal{X})$, which is not a tractable operation, especially since the cardinality of $\mathcal{X}$ (i.e., $N$ ) is random. Moreover, the integral required to obtain the probability is over a region with an irregular boundary. Instead, an effective and pragmatic approach to finding the distribution of $p_{\mathrm{c}}$ is to use a simulation. The simulation involves the repeated realization of the set $\mathcal{X}$. For each realization of $\mathcal{X}$, the resulting $p_{\mathrm{c}}(\mathcal{X})$ is found and recorded. The $\mathrm{CDF}$ of $p_{\mathrm{c}}$ is then found by plotting the empirical CDF of the recorded data.

The connectivity distribution $F_{\mathrm{c}}(\gamma)=\mathbb{P}\left[p_{\mathrm{c}} \leq \gamma\right]$ is shown in Fig. 5.3 for urban and suburban environments, taking into account the two kinds of vehicle locations. In particular, 
the blue dashed lines correspond to the case that the vehicle is located at an intersection, the dotted red lines correspond to the case that the vehicle is located outside an intersection, and the green solid lines account for both cases by averaging them according to 5.12). The distributions are computed by fixing the values of $r_{\max }=250 \mathrm{~m}, \lambda_{\mathrm{UAV}}=20 / \mathrm{km}^{2}$, and $h_{\mathrm{UAV}}=100 \mathrm{~m}$.

When comparing the connectivity distribution with figures such as Fig. 5.3, curves that are relatively lower correspond to better performing scenarios than curves that are higher. This is because the lower curves are less likely to have coverage probabilities that fail to meet a threshold (the value of the argument $\gamma$ ), and hence are more likely to have coverage probabilities that are better than $\gamma$. Thus, Fig. 5.3 shows that performance is consistently better in suburban environments than in urban environments, which can be attributed to the lower value of $\mu_{\mathrm{H}}$ and hence shorter buildings in the suburbs. The figure also shows that performance is better when the vehicle is at intersections than when it is not at an intersection. This is due to the fact that, at an intersection, the vehicle can find a LoS path directly down two streets instead of just down the one street. When looking at the combined probability, we see that the performance is closer to the case where it is outside an intersection, since a vehicle moving at constant velocity is more likely to be outside of an intersection than it is to be at an intersection. For the remaining results in this section, the connection probability is found by averaging the probabilities at the two vehicle locations.

Fig. 5.4 shows the connectivity distribution for two different values of the maximum transmission range, $r_{\max }=\{200,300\} \mathrm{m}$, taking into account all three different city types. When $r_{\max }$ is greater, the CDF is lower, due to the fact that the vehicle is likely to have more UAVs within its range. The CDF also varies depending on the deployment, since building heights are generally higher for urban deployments and even higher for dense urban deployments, hence the denser environments perform worse than the sparser ones.

Figs. 5.5 and 5.6 show the outage probability rather than the connectivity distribution. The outage probability is merely the value of the connectivity distribution at a specified threshold $\gamma_{\mathrm{th}}$. Hence, the outage probability is $F_{\mathrm{c}}\left(\gamma_{\mathrm{th}}\right)$ and quantifies the probability that a given network realization fails to have a coverage probability of $\gamma_{\text {th }}$. For these curves, $\gamma_{\text {th }}=0.8$. Fig. 5.5 shows the outage probability as a function of UAV height in an urban 


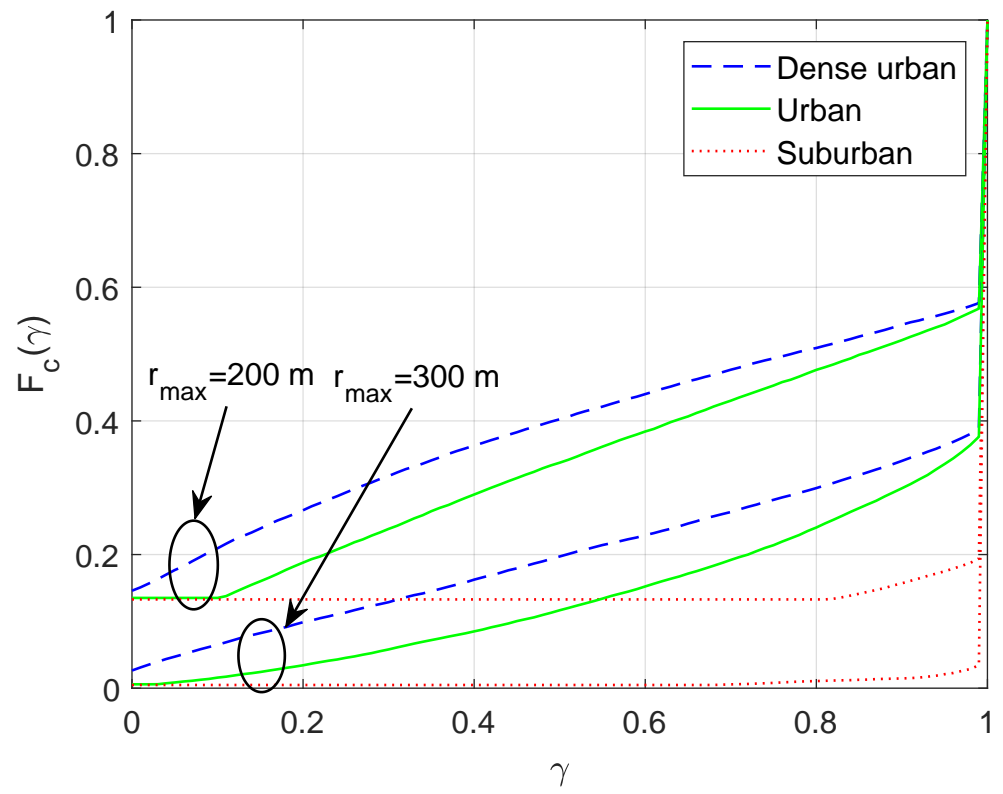

Figure 5.4: The connectivity distribution when $r_{\max }=200 \mathrm{~m}$ and $300 \mathrm{~m}$ for dense urban, urban, and suburban deployments. UAV density and UAV height are fixed at values of $\lambda_{\mathrm{UAV}}=20 / \mathrm{km}^{2}, h_{\mathrm{UAV}}=100 \mathrm{~m}$.

environment for three different values of UAV density $\lambda_{\mathrm{UAV}}=\{10,20,30\} / \mathrm{km}^{2}$ with $r_{\max }=$ $250 \mathrm{~m}$. The figure shows that there is an optimal value of the UAV height $h_{\mathrm{UAV}}$. If $h_{\mathrm{UAV}}$ is too low, it will be more easily blocked by buildings that are tall enough to intersect the LoS path. However, if $h_{\text {UAV }}$ is too high, the projection $d_{\max }$ onto the ground plane will get smaller due to the geometry (see Fig. 2), and thus the average number of UAVs within range will be reduced. The optimal height increases with decreasing UAV density, suggesting that when the UAV density is low, it is more important for close UAV to clear nearby buildings by being sufficiently high than it is for there to be a large area containing UAVs.

Fig. 5.6 shows the outage probability as a function of UAV height for all three city types at UAV density $\lambda_{\text {UAV }}=20 / \mathrm{km}^{2}$ with $r_{\max }=250 \mathrm{~m}$. The figure shows that the optimal height depends on the type of city environment, since denser city environments have a taller mean building height. As the city environment gets denser, higher UAV altitudes are required to overcome blocking by the taller buildings.

Fig. 5.7 is a contour plot that shows contours of constant outage probability as a function of both UAV density $\lambda_{\mathrm{UAV}}$ and UAV height $h_{\mathrm{UAV}}$. Contours are spaced in increments of 0.1 , with 0.1 being the top curve and 0.9 the bottom curve. The outage threshold is $\gamma_{\text {th }}=0.8$, 


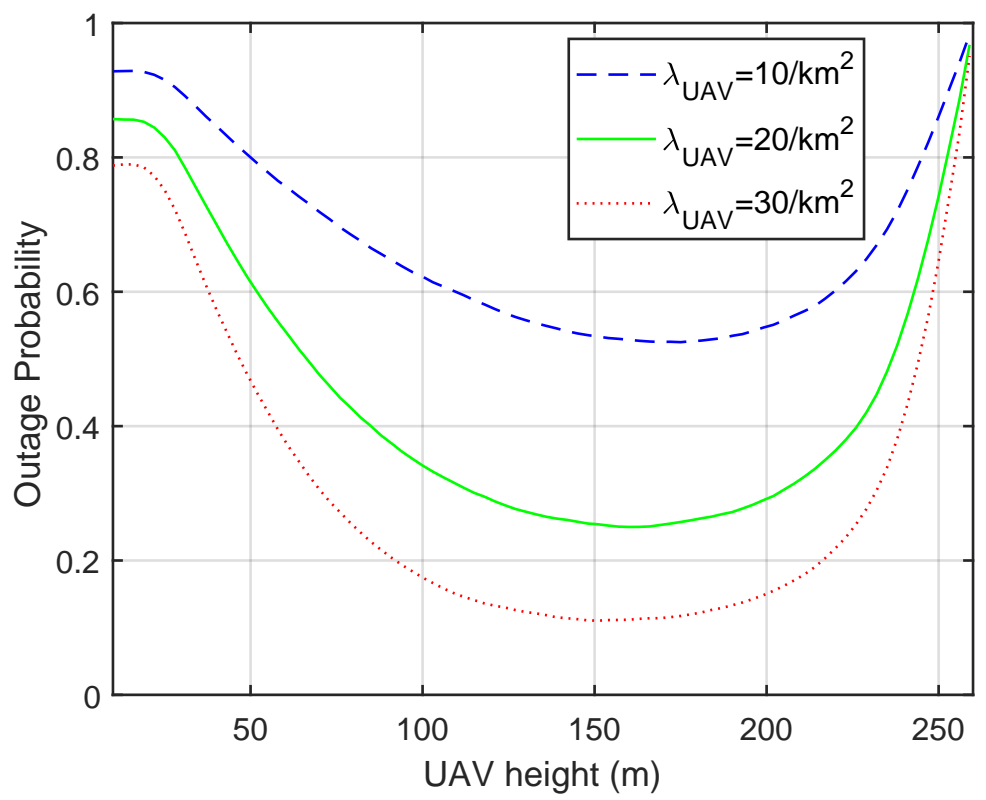

Figure 5.5: The outage probability at threshold $\gamma_{\text {th }}=0.8$ with respect to $h_{\text {UAV }}$ when $\lambda_{\text {UAV }}=$ $10,20,30 / \mathrm{km}^{2}$. The deployment is urban and the maximum transmission range is fixed at $r_{\max }=250 \mathrm{~m}$.

the city type is urban, and the maximum transmission range is $r_{\max }=250 \mathrm{~m}$. The figure can be used to determine the minimum UAV density and optimal height required to meet an outage constraint. For instance, if the system must operate at an outage of 0.1, then the curve shows that the UAV density must be at least $\lambda_{\mathrm{UAV}}=31 / \mathrm{km}^{2}$ and the height should be $h_{\text {UAV }}=162 \mathrm{~m}$ when the minimum UAV density used.

Figs. 5.9 and 5.10 show the result of the optimization given by 5.13 for a threshold of $\gamma_{\mathrm{th}}=0.8$ and $r_{\max }=250 \mathrm{~m}$. In particular, Fig. 5.9 shows the optimal UAV height as a function of UAV density for each of the three city types, and Fig. 5.10 shows the corresponding outage probability. From these curves, it is again observed that the optimal height decreases with increasing UAV density and that the optimal height is lower for less dense city environments. Together, these curves can be used to determine the minimum UAV density required to achieve a desired outage probability (by reading Fig. 5.10) and the corresponding optimal UAV height (by reading from Fig. 5.9). 


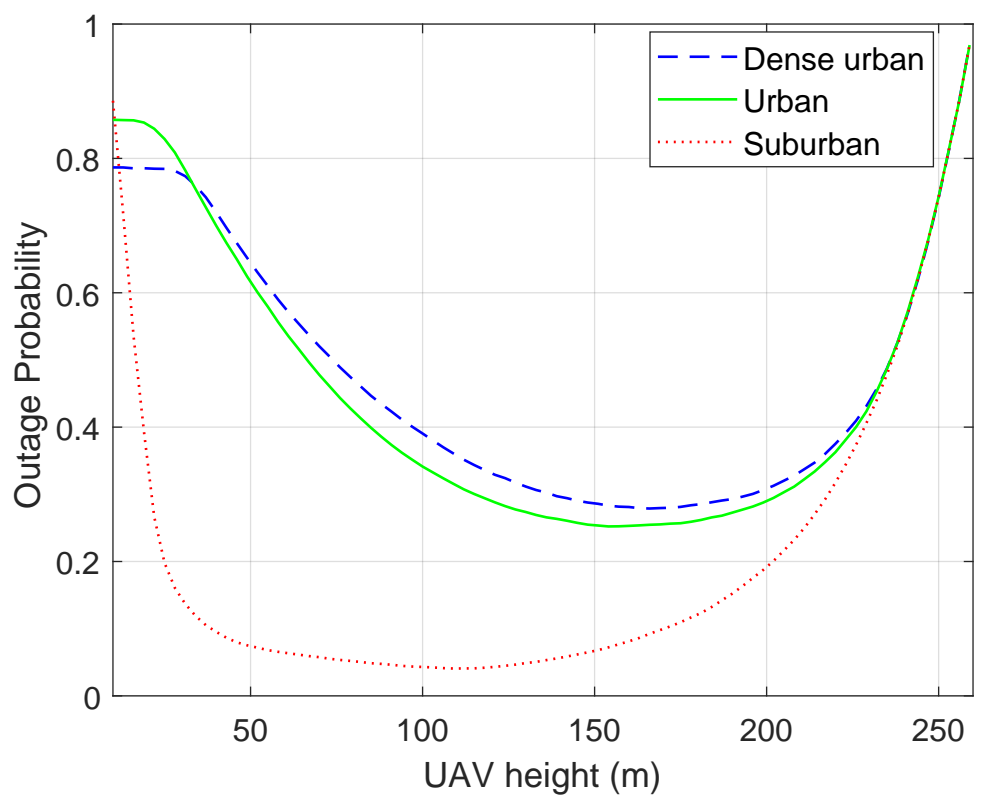

Figure 5.6: The outage probability at threshold $\gamma_{\mathrm{th}}=0.8$ with respect to $h_{\mathrm{UAV}}$ for the three city types. UAV density and maximum transmission range are fixed at values of $\lambda_{\mathrm{UAV}}=20 / \mathrm{km}^{2}$ and $r_{\max }=250 \mathrm{~m}$.

\subsection{Conclusion}

By using a MPLP to model the locations of city streets and taking into account the random heights of the buildings, the methodology in this chapter can be used to compute the connectivity probability for a randomly deployed network of multiple UAVs. For UAVs at fixed location, the conditional connectivity probability characterizes the connectivity conditioned on those locations. When the UAVs are drawn from a point process, then removing the conditioning allows the connectivity to be characterized by its distribution, which gives the probability that a network realization (set of UAV locations) fails to meet a threshold. The connectivity distribution can then be used to find the outage probability, which is the distribution evaluated at a specified outage threshold. With the outage probability so defined, it can be used to characterize the tradeoff between UAV density and network performance for different city types. Moreover, the outage probability can be used to drive an optimization that identifies the optimal UAV height.

We note some limitations of this work that imply directions for future work. We assume that blocking is independent, but in reality it is likely that a large building may block 


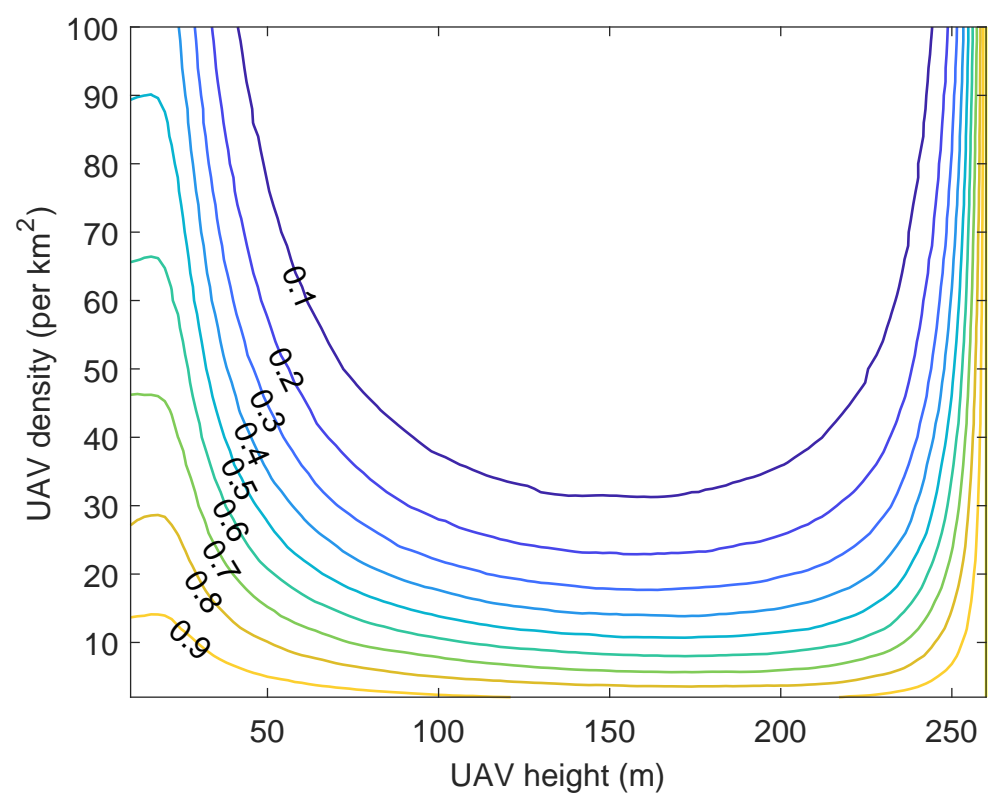

Figure 5.7: Contour plot showing the outage probability as a function of both UAV density $\lambda_{\text {UAV }}$ and UAV height $h_{\text {UAV }}$. Contours are spaced in increments of 0.1 , with 0.1 being the topmost curve.

several UAVs simultaneously. The extent of this issue could be initially explored through simulation, and if correlated blocking proves to significantly impact performance, then the analysis should be extended to account for it. Such an extension is beyond the scope of this chapter, but could potentially avail of the approximations provided in [44]. With respect to optimization, we assume that all UAVs are at a constant altitude, but it may prove to be beneficial for UAVs to be at different altitudes. Instead of optimizing a fixed altitude, the optimization could be used to identify an optimizing altitude distribution. Finally, we note that the physical-layer model is captured by a single parameter - the maximum transmission range - but a more detailed analysis could explicitly capture the effects of fading, interference, and antenna beamforming. Such analysis could also capture the effects of building reflections.

While this chapter has focused on mmWave transmission, it applies equally well to other modes of communication that are primarily LoS, such as free-space optical (FSO) communication. 


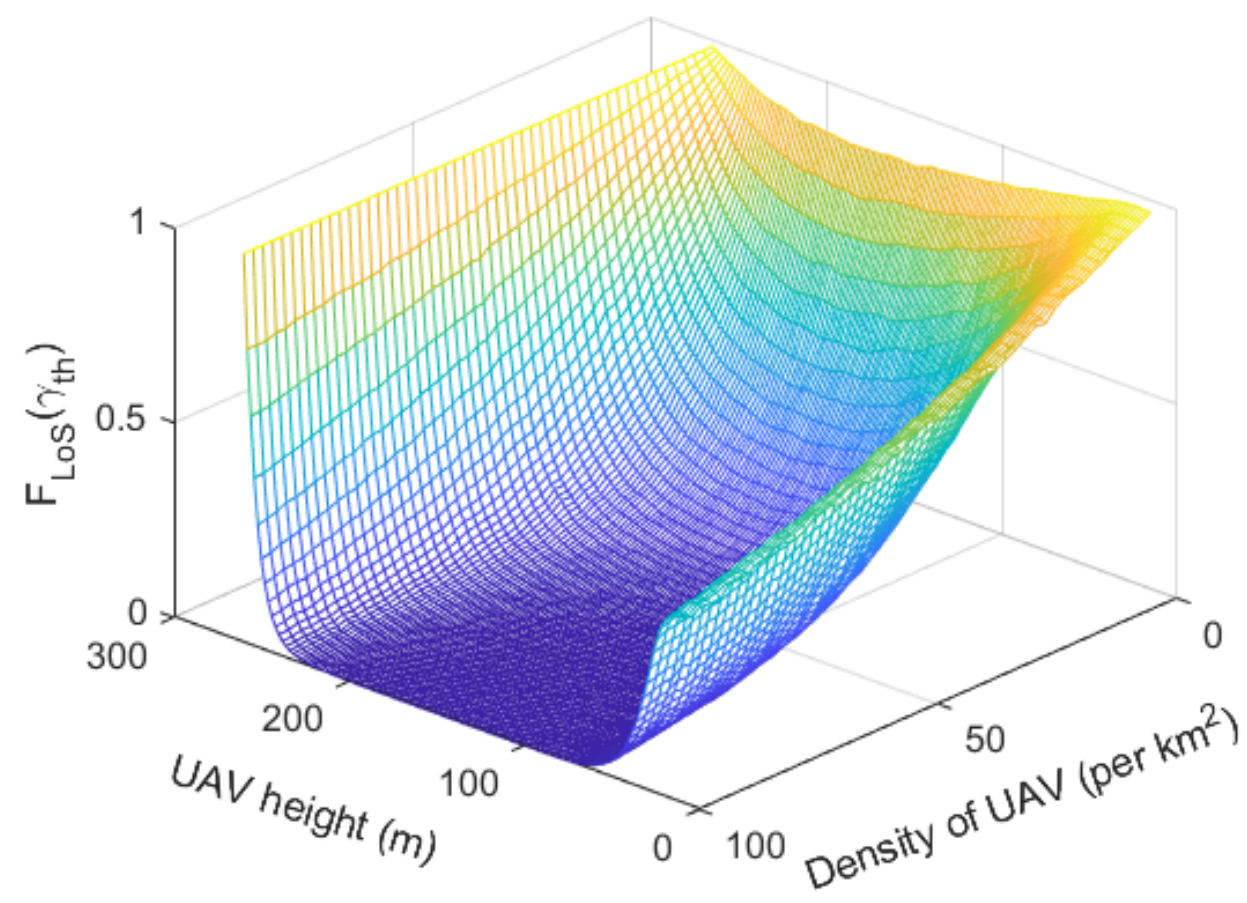

Figure 5.8: 3D Contour plot showing the outage probability as a function of both UAV density $\lambda_{\text {UAV }}$ and UAV height $h_{\text {UAV }}$.

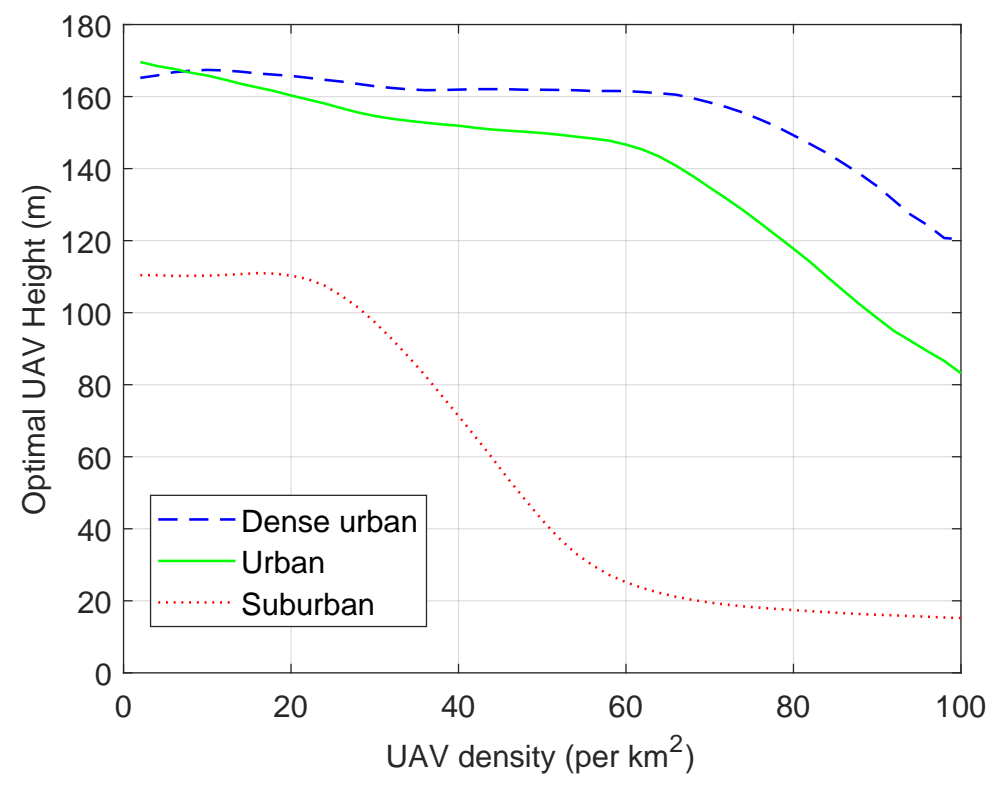

Figure 5.9: The optimal UAV height with respect to $\lambda_{U A V}$ for dense urban, urban, and suburban deployments. 


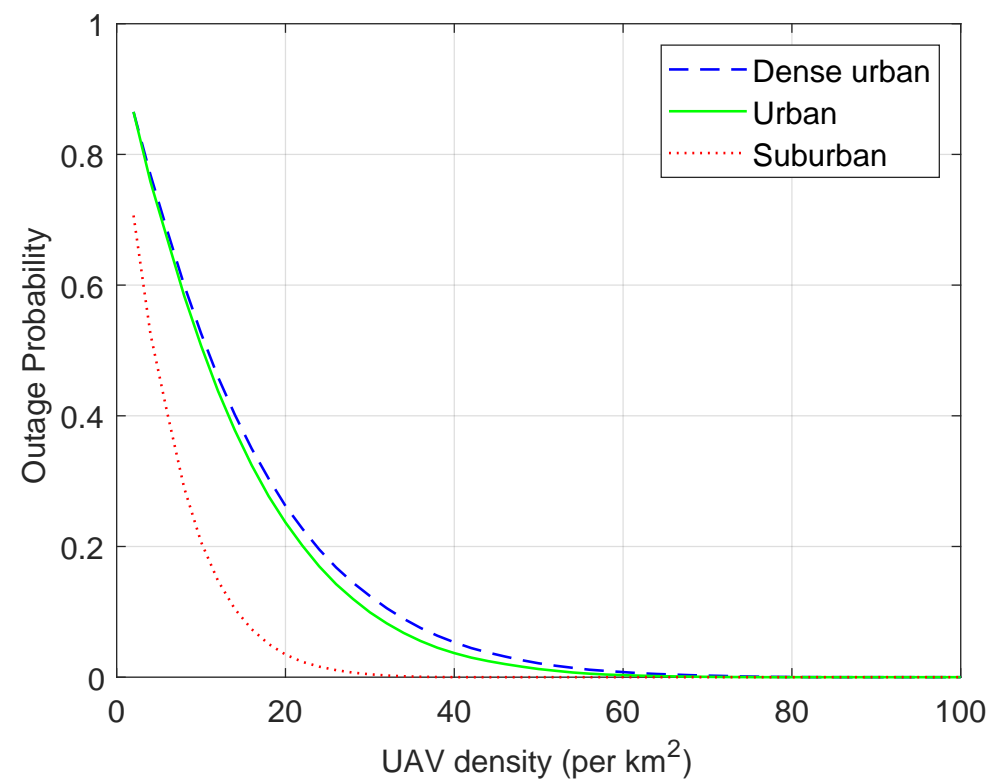

Figure 5.10: Outage probability achieved when the optimal UAV height is used in dense urban, urban, and suburban deployments. 


\section{Chapter 6}

\section{Contributions and Further Research}

\subsection{Summary}

This chapter provides outline that summarises the general conclusions drawn from this thesis and provides some future research directions. The dissertation deals with understanding mmWave networks, which are poised to be a fundamental component in the current and future wireless world. This dissertation developed a set of new frameworks to characterize performance of mmWave networks, as follows:

1. It provides an exact evaluation of SINR outage of networks consisting of user devices and independent blocking with fixed and random locations.

2. It carefully characterizes correlated blocking and analyzes its impact on the performance of mmWave networks.

3. It identifies macrodiversity as an important strategy to mitigating blocking in mmWave networks in the presence of correlated random blocking and interference, and performs the corresponding analysis.

4. It characterizes and optimizes the performance of UAV to ground networks operating at mmWave frequencies by analyzing the connectivity probability as a function of the city type (e.g., urban, dense urban, suburban), density of UAVs, and height of the UAVs. 


\subsection{Research Contributions}

A complete list of the contributions of the dissertation is as follows.

- Analyzing SINR outage in mmWave Device-to-Device networks operating in finite-sized crowds.

1. The dissertation develops a framework to accurately model human body blockage, antenna parameters, and key propagation features of mmWave communication.

2. It derives an exact expression for SINR outage conditioned on the location of human users and interferers.

3. It analyzes spatially averaged results via closed-form expressions using justifiable assumptions.

- Developing a tractable model to predict the impact of correlated blocking on the performance of mmWave personal networks.

1. The dissertation derives a closed form expressions for blockage correlation coefficient and the distribution of SINR are provided for the case of two dominant interferers and fixed number of blockages.

2. It analyzes the effect of antenna directivity and the spatial randomness of the interferers.

3. It provides insight into the validity of the commonly held assumption of independent blocking and the improved accuracy that can be obtained when the blocking correlation is taken into account.

- Introducing macrodiversity technique to mitigate the effect of blocking in mmWave networks.

1. The dissertation develops a framework to determine distributions for the LOS probability, Signal to Noise Ratio (SNR), and Signal to Interference and Noise Ratio (SINR) for macrodiverity cellular networks by taking into account correlated blocking. 
2. It validates the framework by comparing the analysis, which models blockages using a random point process, with an analysis that uses real-world data to account for blockage.

3. It studies the impact of blockage size and blockage density along with the effect of co-channel interference arising from other cells. We show that the assumption of independent blocking can lead to an incorrect evaluation of macrodiversity gain, as the correlation tends to decrease macrodiversity gain.

4. It extends the study to more general case of an arbitrary macrodiversity order, and identify the minimum macrodiversity order required to achieve desired performance in the presence of interference.

- Identifying the connectivity probability of UAV to ground networks in urban environments.

1. The dissertation develops a framework to characterize the connectivity probability as a function of the city type (e.g., urban, dense urban, suburban), density of UAVs, and height of the UAVs.

2. It finds the outage probability from the connectivity distribution. With the outage probability so defined, it is used to characterize the tradeoff between UAV density and network performance for different city types.

3. It provides an optimization of UAV to ground networks by identifying the optimal UAV height that minimizes the outage probability for different urban deployments.

\subsection{Conclusions and Future Research Directions}

In Chapter 2, we found analytical expressions that exactly characterize the outage probability in wireless networks when the power of each interferer is selected at random. The set of distributions can correspond to different blockage and directivity states, making it immediately applicable to mmWave systems. 
The work could readily be extended to processes other than BPPs, such as Poisson point processes, as well as to networks of shapes other than an annulus. A more detailed analysis could use these expressions to provide insight into the role of various parameters such as the number of interferers $(K)$, channel coefficients, and SNR. While the focus has been on mmWave, other applications are possible related to cellular networks, distributed MIMO systems, and more elaborate MAC protocols. For instance, the same methodology could be used to model channel access schemes with various types of collisions (e.g, full and partial) each with their own severity and probability.

In Chapter 3 the focus is correlated blocking in mmWave WPAN system, which can occur, for instance, when a single blockage to block multiple interferers if the blockage is sufficiently wide and the interferers sufficiently close. By using the proposed methodology, the correlation between two sources of interference may be found and factored into the analysis, yielding more accurate results than the assumptions of independent blocking.

The analysis can be extended in a variety of ways. We have already shown that it can be combined with an analysis that accounts for antenna directivity and the random location and orientation of the interferers. While the chapter has focused on the extreme case that LOS signals are AWGN while NLOS signals are completely blocked, it is possible to adapt the analysis to more sophisticated channels [14]. For instance, the use of different fading and path-loss parameters for LOS and NLOS states was considered in Chapter 2, and could be combined with the results in Chapter 3.

Finally, while the chapter focused on the pairwise correlation between two interferers, it can be extended to the more general case of an arbitrary number of interferers. One way to handle this is to only consider the correlation of the two closest interferers (the most dominant ones) while assuming that all other interferers are subject to independent blocking. Another solution is to group interferers into pairs, and only consider the pairwise correlation, while neglecting higher order effects. As performance in a mmWave system is typically dominated by just a few interferers, we anticipate that either of these approaches would yield accurate results. We also anticipate that when several interferers are present, the effects of correlation will be even more pronounced in a random network, as the likelihood that two interferers are close together increases with the number of interferers. 
In Chapter 4. We have proposed a framework to analyze the second-order macrodiversity gain for mmWave cellular system in the presence of correlated blocking. The chapter considered the distributions of LOS probability, SNR, and, when there is interference, the SINR. The framework was confirmed by comparing the analysis to a real data model.

The analysis can be extended in a variety of way. While the chapter has focused on the extreme case that LOS signals are AWGN while NLOS signals are completely blocked, it is possible to adapt the analysis to more sophisticated channels, such as those where both LOS and NLOS signals are subject to fading and path loss, but the fading and path loss parameters are different depending on the blocking state as was considered in Chapter 2 . Moreover, the study can be extended to the more general case of an arbitrary macrodiversity order; i.e., diversity orders greater than two. Such a study could identify the minimum macrodiversity order required to achieve desired performance in the presence of interference. We anticipate that when more than two base stations are connected, the effects of correlation on macrodiversity gain will increase and the negative impact of interference will decrease. This is because the likelihood that two base stations are close together increases with the number of base stations and the ratio of the number of connected base stations to the number of interfering transmitters will increase.

The methodology in Chapter 5, can be used to compute the connectivity probability for a randomly deployed network of multiple UAVs. The connectivity distribution can be used to find the outage probability, with the outage probability so defined, it can be used to characterize the tradeoff between UAV density and network performance for different city types. Moreover, the outage probability can be used to drive an optimization that identifies the optimal UAV height.

We note some limitations of the work of Chapter 5 that imply directions for future work. We assume that blocking is independent, but in reality it is likely that a large building may block several UAVs simultaneously. The extent of this issue could be initially explored through simulation, and if correlated blocking proves to significantly impact performance, then the analysis should be extended to account for it. Such an extension is beyond the scope of the chapter, but could potentially avail of the approximations provided in [44]. With respect to optimization, we assume that all UAVs are at a constant altitude, but it 
may prove to be beneficial for UAVs to be at different altitudes. Instead of optimizing a fixed altitude, the optimization could be used to identify an optimizing altitude distribution. Finally, we note that the physical-layer model is captured by a single parameter - the maximum transmission range - but a more detailed analysis could explicitly capture the effects of fading, interference, and antenna beamforming. Such analysis could also capture the effects of building reflections. The analysis could be also extended to more irregular urban environments, one where it is not a regular Manhattan-like grid arrangement of streets. While this dissertation has focused on mmWave transmission, the results that it develops may be applicable to other modes of communication that are primarily LoS, such as free-space optical (FSO) communication. 


\section{Appendix A}

\section{A Derivation of the PMF of a Pair of Correlated Bernoulli Variables}

As in [61], the correlation coefficient between $B_{1}$ and $B_{2}$ is given by

$$
\rho=\frac{E\left[B_{1} B_{2}\right]-E\left[B_{1}\right] E\left[B_{2}\right]}{\sqrt{\sigma_{B_{1}}^{2} \sigma_{B_{2}}^{2}}}
$$

where the expected value and the variance of the Bernoulli variable $B_{i}$ is given by 61

$$
\begin{aligned}
E\left[B_{i}\right] & =p_{i} \\
\sigma_{B_{i}}^{2} & =p_{i} q_{i} .
\end{aligned}
$$

By substituting A.2 and A.3 into A.1 and solving for $E\left[B_{1} B_{2}\right]$,

$$
E\left[B_{1} B_{2}\right]=p_{1} p_{2}+\rho \sqrt{p_{1} p_{2} q_{1} q_{2}}=p_{1} p_{2}+\rho h .
$$

As in [61], we can relate $p_{B_{1}, B_{2}}\left(b_{1}, b_{2}\right)$ to $E\left[B_{1} B_{2}\right]$ as follows:

$$
E\left[B_{1} B_{2}\right]=\sum_{b_{1}} \sum_{b_{2}} b_{1} b_{2} p_{B_{1}, B_{2}}\left(b_{1}, b_{2}\right)=p_{B_{1}, B_{2}}(1,1),
$$

where solving the sum relies there being only one nonzero value for $b_{1} b_{2}$. By solving for $p_{B_{1}, B_{2}}(1,1)$ and using A.4.

$$
p_{B_{1}, B_{2}}(1,1)=p_{1} p_{2}+\rho h .
$$


We can relate $p_{B_{1}, B_{2}}\left(b_{1}, b_{2}\right)$ to $E\left[B_{1}\right]$ as follows:

$$
\begin{aligned}
E\left[B_{1}\right] & =\sum_{b_{1}} \sum_{b_{2}} b_{1} p_{B_{1}, B_{2}}\left(b_{1}, b_{2}\right) \\
& =p_{B_{1}, B_{2}}(1,1)+p_{B_{1}, B_{2}}(1,0) .
\end{aligned}
$$

Solving for $p_{B_{1}, B_{2}}(1,0)$,

$$
p_{B_{1}, B_{2}}(1,0)=E\left[B_{1}\right]-p_{B_{1}, B_{2}}(1,1)=p_{1} q_{2}-\rho h
$$

Similarly, it can be shown that

$$
p_{B_{1}, B_{2}}(0,1)=q_{1} p_{2}-\rho h .
$$

Finally, since 61]

$$
\sum_{b_{1}} \sum_{b_{2}} p_{B_{1}, B_{2}}\left(b_{1}, b_{2}\right)=1
$$

it follows that

$$
\begin{aligned}
p_{B_{1}, B_{2}}(0,0) & =1-p_{B_{1}, B_{2}}(1,0)-p_{B_{1}, B_{2}}(0,1)-p_{B_{1}, B_{2}}(1,1) \\
& =q_{1} q_{2}+\rho h .
\end{aligned}
$$

In conclusion, the joint pmf is:

$$
p_{B_{1}, B_{2}}\left(b_{1}, b_{2}\right)= \begin{cases}q_{1} q_{2}+\rho h & \text { for } b_{1}=0, b_{2}=0 \\ q_{1} p_{2}-\rho h & \text { for } b_{1}=0, b_{2}=1 \\ p_{1} q_{2}-\rho h & \text { for } b_{1}=1, b_{2}=0 \\ p_{1} p_{2}+\rho h & \text { for } b_{1}=1, b_{2}=1\end{cases}
$$

where $h=\sqrt{p_{1} p_{2} q_{1} q_{2}}$. 


\section{Appendix B}

\section{A Derivation of Distances to the Closest Transmitters}

As in [44], the pdf of the smallest distance $R_{1}$ is

$$
f\left(r_{1}\right)=2 \pi \lambda r_{1} e^{-\lambda \pi r_{1}^{2}}
$$

for $r_{1} \geq 0$. From (B.1), we can derive the conditional CDF of $R_{i}$ given $R_{i-1}$ as

$$
F_{R_{i}}\left(r_{i} \mid R_{i-1}=r_{i-1}\right)=1-e^{\lambda \pi\left(r_{i}^{2}-r_{i-1}^{2}\right)}
$$

To generate random variables $r_{1}, \ldots, r_{N}$, let $x_{i} \sim U(0,1)$,

$$
x_{i}=F_{R_{i}}\left(r_{i} \mid R_{i-1}=r_{i-1}\right)=1-e^{\lambda \pi\left(r_{i}^{2}-r_{i-1}^{2}\right)}
$$

Solving for $r_{i}$,

$$
r_{i}=\sqrt{-\frac{1}{\lambda \pi} \ln \left(1-x_{i}\right)+r_{i-1}^{2}}
$$

where $r_{0}=0$. Start by generating $x_{i}$ as uniform random variables, then recursively substitute each one in (B.4) to get the desired random variable $r_{i}$. 


\section{References}

[1] C. Seker, M. T. Güneser, and T. Ozturk, "A review of millimeter wave communication for 5g," in Proc. International Symposium on Multidisciplinary Studies and Innovative Technologies (ISMSIT), Oct. 2018.

[2] T. Rappaport et al, "Millimeter wave mobile communications for 5G cellular: It will work!," IEEE Access, vol. 1, pp. 335-349, 2013.

[3] M. R. Akdeniz, Y. Liu, M. K. Samimi, S. Sun, S. Rangan, T. S. Rappaport, and E. Erkip, "Millimeter wave channel modeling and cellular capacity evaluation," IEEE Journal on Selected Areas in Communications, vol. 32, pp. 1164-1179, June 2014.

[4] X. Wang, L. Kong, F. Kong, F. Qiu, M. Xia, S. Arnon, and G. Chen, "Millimeter wave communication: A comprehensive survey," IEEE Communications Surveys Tutorials, vol. 20, no. 3, pp. 1616-1653, 2018.

[5] T. Bai, R. Vaze, and R. W. Heath, Jr., "Analysis of blockage effects on urban cellular networks," IEEE Transactions on Wireless Communications, vol. 13, pp. 5070-5083, Sept. 2014.

[6] S. Tripathi, N. V. Sabu, A. K. Gupta, and H. S. Dhillon, "Millimeter-wave and terahertz spectrum for 6g wireless," CoRR, vol. abs/2102.10267, 2021.

[7] T. Rappaport, R. W. Heath, Jr., R. C. Daniels, and J. N. Murdock, Millimeter Wave Wireless Communications. Pearson Education, 2014.

[8] E. Hriba and M. C. Valenti, "Correlated blocking in mmwave cellular networks: Macrodiversity, outage, and interference," Electronics (Special Issue on Millimeter-Wave (mmWave) Communications), Oct. 2019.

[9] R. Fisher, "60 GHz WPAN standardization within IEEE 802.15.3c," in Proc. International Symposium on Signals, Systems and Electronics (ISSSE), pp. 103-105, July 2007.

[10] S. Singh, F. Ziliotto, U. Madhow, E. Belding, and M. Rodwell, "Blockage and directivity in $60 \mathrm{GHz}$ wireless personal area networks: From cross-layer model to multihop MAC design," IEEE Journal on Selected Areas in Communications, vol. 27, pp. 1400-1413, Oct. 2009. 
[11] Y. Niu, Y. Li, D. Jin, L. Su, and D. Wu, "Blockage robust and efficient scheduling for directional mmWave WPANs," IEEE Transactions on Vehicular Technology, vol. 64, pp. 728-742, Feb. 2015.

[12] K. Venugopal, M. C. Valenti, and R. W. Heath, Jr., "Interference in finite-sized highly dense millimeter wave networks," in Proc. Information Theory and Applications Workshop (ITA), pp. 175-180, Feb. 2015.

[13] K. Venugopal, M. C. Valenti, and R. W. Heath, Jr., "Device-to-device millimeter wave communications: Interference, coverage, rate, and finite topologies," IEEE Transactions on Wireless Communications, vol. 15, pp. 6175-6188, Sept. 2016.

[14] E. Hriba, M. C. Valenti, K. Venugopal, and R. W. Heath, Jr., "Accurately accounting for random blockage in device-to-device mmWave networks," in Proc. IEEE Global Communications Conference (GLOBECOM), Dec. 2017.

[15] S. Aditya, H. S. Dhillon, A. F. Molisch, and H. Behairy, "Asymptotic blind-spot analysis of localization networks under correlated blocking using a Poisson line process," IEEE Wireless Communications Letters, vol. 6, pp. 654-657, Oct. 2017.

[16] S. Aditya, H. S. Dhillon, A. F. Molisch, and H. M. Behairy, "A tractable analysis of the blind spot probability in localization networks under correlated blocking," IEEE Transactions on Wireless Communications, vol. 17, no. 12, pp. 8150-8164, 2018.

[17] B. Shang, L. Liu, J. Ma, and P. Fan, "Unmanned aerial vehicle meets vehicle-toeverything in secure communications," IEEE Communications Magazine, vol. 57, pp. 98103, Oct. 2019.

[18] C. T. Cicek, H. Gultekin, B. Tavli, and H. Yanikomeroglu, "UAV base station location optimization for next generation wireless networks: Overview and future research directions," in Proc. 1st International Conference on Unmanned Vehicle Systems (UVS), 2019.

[19] C.-H. Liu, K.-H. Ho, and J.-Y. Wu, "Mmwave UAV networks with multi-cell association: Performance limit and optimization," IEEE Journal on Selected Areas in Communications, vol. 37, pp. 2814-2831, Dec. 2019.

[20] X. He, W. Yu, H. Xu, J. Lin, X. Yang, C. Lu, and X. Fu, "Towards 3D deployment of UAV base stations in uneven terrain," in Proc. International Conference on Computer Communication and Networks (ICCCN), 2018.

[21] M. Kishk, A. Bader, and M.-S. Alouini, "Aerial base station deployment in 6G cellular networks using tethered drones: The mobility and endurance tradeoff," IEEE Vehicular Technology Magazine, vol. 15, pp. 103-111, Dec. 2020.

[22] E. Hriba and M. C. Valenti, "The potential gains of macrodiversity in mmwave cellular networks with correlated blocking," in Proc. IEEE Military Communications Conference (MILCOM), Nov. 2019. 
[23] K. Han, K. Huang, Y. Cui, and Y. Wu, "The connectivity of millimeter-wave networks in manhattan-type regions," in Proc. IEEE Global Communications Conference (GLOBECOM), Dec. 2017.

[24] F. Baccelli and B. Blaszczyszyn, Stochastic Geometry and Wireless Networks - Volume I: Theory, vol. 3. Paris: Now, 2009.

[25] F. Baccelli and B. Blaszczyszyn, Stochastic Geometry and Wireless Networks - Volume II: Applications, vol. 4. Paris: Now, 2009.

[26] M. Haenggi, Stochastic Geometry for Wireless Networks. Cambridge University Press, 2012.

[27] T. Bai and R. W. Heath, Jr., "Coverage and rate analysis for millimeter-wave cellular networks," IEEE Transactions on Wireless Communications, vol. 14, pp. 1100-1114, Feb. 2015 .

[28] S. Singh, M. N. Kulkarni, A. Ghosh, and J. G. Andrews, "Tractable model for rate in self-backhauled millimeter wave cellular networks," IEEE Journal on Selected Areas in Communications, vol. 33, pp. 2196-2211, Oct. 2015.

[29] M. Di Renzo, "Stochastic geometry modeling and analysis of multi-tier millimeter wave cellular networks," IEEE Transactions on Wireless Communications, vol. 14, pp. 50385057, Sept. 2015.

[30] J. G. Andrews, T. Bai, M. N. Kulkarni, A. Alkhateeb, A. K. Gupta, and R. W. Heath, Jr., "Modeling and analyzing millimeter wave cellular systems," IEEE Transactions on Communications, vol. 65, pp. 403-430, Jan. 2017.

[31] J. Zhang and J. Andrews, "Distributed antenna systems with randomness," IEEE Transactions on Wireless Communications, vol. 7, pp. 3636-3646, September 2008.

[32] G. George, K. Venugopal, A. Lozano, and R. W. Heath, "Enclosed mmwave wearable networks: Feasibility and performance," IEEE Transactions on Wireless Communications, vol. 16, no. 4, pp. 2300-2313, 2017.

[33] M. C. Valenti, D. Torrieri, and S. Talarico, "Adjacent-channel interference in frequencyhopping ad hoc networks," in Proc. IEEE International Conference on Communications (ICC), pp. 5583-5588, Jun. 2013.

[34] D. Torrieri, S. Talarico, and M. C. Valenti, "Analysis of a frequency-hopping millimeterwave cellular uplink," IEEE Transactions on Wireless Communications, vol. 15, pp. 7089-7098, Oct. 2016.

[35] M. C. Valenti, D. Torrieri, and S. Talarico, "A direct approach to computing spatially averaged outage probability," IEEE Communications Letters, vol. 18, pp. 1103-1106, July 2014. 
[36] J. Mo, B. L. Ng, S. Chang, P. Huang, M. N. Kulkarni, A. Alammouri, J. C. Zhang, J. Lee, and W. Choi, "Beam codebook design for 5G mmwave terminals," IEEE Access, vol. 7, pp. 98387-98404, 2019.

[37] J. F. Harvey, M. B. Steer, and T. S. Rappaport, "Exploiting high millimeter wave bands for military communications, applications, and design," IEEE Access, vol. 7, pp. 52350$52359,2019$.

[38] C. Chen, O. Kedem, C. R. C. M. d. Silva, and C. Cordeiro, "Millimeter-wave fixed wireless access using IEEE 802.11ay," IEEE Communications Magazine, vol. 57, no. 12, pp. 98-104, 2019.

[39] R. Fontana and P. Semeraro, "Characterization of multivariate Bernoulli distributions with given margins," arXiv:1706.01357[math.ST], June 2017.

[40] E. Anderson, G. Yee, C. Phillips, D. Sicker, and D. Grunwald, "The impact of directional antenna models on simulation accuracy," in Proc. Yth International Symposium on Modeling and Optimization in Mobile, Ad Hoc, and Wireless Networks, June 2009.

[41] J. Park, J. G. Andrews, and R. W. Heath, Jr., "Inter-operator base station coordination in spectrum-shared millimeter wave cellular networks," IEEE Transactions on Cognitive Communications and Networking, vol. 4, pp. 513-528, Sept. 2018.

[42] S. Singh, M. N. Kulkarni, A. Ghosh, and J. G. Andrews, "Tractable model for rate in self-backhauled millimeter wave cellular networks," IEEE Journal on Selected Areas in Communications, vol. 33, pp. 2196-2211, Oct. 2015.

[43] J. Choi, "On the macro diversity with multiple bss to mitigate blockage in millimeterwave communications," IEEE Communications Letters, vol. 18, pp. 1653-1656, Sept. 2014.

[44] A. K. Gupta, J. G. Andrews, and R. W. Heath, Jr., "Macrodiversity in cellular networks with random blockages," IEEE Transactions on Wireless Communications, vol. 17, pp. 996-1010, Feb. 2018.

[45] A. K. Gupta, J. G. Andrews, and R. W. Heath, Jr., "Impact of correlation between link blockages on macro-diversity gains in mmwave networks," in Proc. IEEE International Conference on Communications Workshops (ICC Workshops), May 2018.

[46] E. Hriba and M. C. Valenti, "The impact of correlated blocking on millimeter-wave personal networks," in Proc. IEEE Military Communications Conference (MILCOM), Oct. 2018.

[47] A. Thornburg and R. W. Heath, Jr., "Ergodic rate of millimeter wave ad hoc networks," IEEE Transactions on Wireless Communications, vol. 17, pp. 914-926, Feb. 2018.

[48] F. Baccelli, B. Błaszczyszyn, and P. Mühlethaler, "An Aloha protocol for multihop mobile wireless networks," IEEE Transactions on Information Theory, vol. 52, pp. 421436, Feb. 2006. 
[49] M. Shi, K. Yang, Z. Han, and D. Niyato, "Coverage analysis of integrated sub-6ghzmmwave cellular networks with hotspots," IEEE Transactions on Communications, vol. 67, pp. 8151-8164, Nov. 2019.

[50] Y. Gao, S. Yang, S. Wu, M. Wang, and X. Song, "Coverage probability analysis for mmwave communication network with ABSF-based interference management by stochastic geometry," IEEE Access, vol. 7, pp. 133572-133582, 2019.

[51] W. A. Gulzar Khawaja, O. Ozdemir, F. Erden, I. Guvenc, M. Ezuma, and Y. Kakishima, "Effect of passive reflectors for enhancing coverage of 28 ghz mmwave systems in an outdoor setting," in Proc. IEEE Radio and Wireless Symposium (RWS), pp. 1-4, 2019.

[52] S. Talarico, M. C. Valenti, and M. Di Renzo, "Outage correlation in finite and clustered wireless networks," in Proc. IEEE International Symposium on Personal, Indoor and Mobile Radio Communications (PIMRC), Sept. 2018.

[53] A. Samuylov, M. Gapeyenko, D. Moltchanov, M. Gerasimenko, S. Singh, N. Himayat, S. Andreev, and Y. Koucheryavy, "Characterizing spatial correlation of blockage statistics in urban mmwave systems," in Proc. IEEE Globecom Workshops, Dec. 2016.

[54] E. Hriba and M. C. Valenti, "Correlated blocking in mmwave cellular networks: Macrodiversity, outage, and interference," in Millimeter-Wave (mmWave) Communications, ch. 11, pp. 137-153, Basel, Switzerland: Springer-Verlag, 2020.

[55] D. L. Snyder and M. I. Miller, Random Point Processes in Time and Space. Springer Texts in Electrical Engineering, 2nd edition, 1991.

[56] A. Goldsmith, Wireless Communications. New York, NY, USA: Cambridge University Press, 2005.

[57] R. Tanbourgi, H. S. Dhillon, J. G. Andrews, and F. K. Jondral, "Effect of spatial interference correlation on the performance of maximum ratio combining," IEEE Transactions on Wireless Communications, vol. 13, pp. 3307-3316, June 2014.

[58] F. Baccelli and X. Zhang, "A correlated shadowing model for urban wireless networks," in Proc. IEEE Conference on Computer Communications (INFOCOM), Apr. 2015.

[59] M. Gapeyenko, D. Moltchanov, S. Andreev, and R. W. Heath, Jr., "Line-of-sight probability for mmwave-based UAV communications in 3D urban grid deployments," IEEE Transactions on Wireless Communications, vol. 20, pp. 6566-6579, Oct. 2021.

[60] E. Hriba and M. C. Valenti, "Optimization of a millimeter-wave uav-to-ground network in urban deployments," in Proc. IEEE Military Communications Conference (MILCOM), Nov. 2021 to appear.

[61] A. Leon-Garcia, Probability and Random Processes For Electrical Engineering. Addison Wesley, 3rd edition, 2008. 